\title{
Fran Ridge Horizontal Coring Summary Report Hole UE-25h\#1, Yucca Mountain Area, Nye County, Nevada
}

\author{
A. E. Norris \\ F. M. Byers, Jr. \\ T.J. Merson
}

\section{DISCLAIMER}

\begin{abstract}
This report was prepared as an account of work sponsored by an agency of the United States Government. Neither the United States (juvernment nor any agency thereof, nor any of their employees, makes any warranty, express or implied, or assumes any legal liability or responsibility for the accuracy, completeness, or usefulness of any information, apparatus, product, or process disclosed, or represents that its use would not infringe privately owned rights. Reference herein to any specific commercial product, process, or service by trade name, trudemark, manufacturer, or otherwise does not necessarily constitute or imply its endorsement, recommendation, or favoring by the United States Government or any agency thereof. The views and opinions of authors expressed herein do not necessarily state or reflect those of the United States Government or any agency thereof.
\end{abstract}
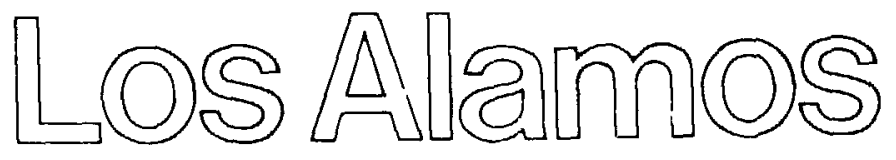

Los Alamos National Laboratory Los Alamos,New Mexico 87545 
FRAN RIDGE HORIZONTAL CORING SLIMMARY REPORT

HOLE UE-25h\#1, YUCCA MOUNTAIN AREA, NYE COUNTY, NEVADA

by

A. E. Norris, F. M. Byers, Jr., and T. J. Merson

\section{ABSTRACT}

Hole UE-25h\#1 was core drilled during December 1982 and January 1983 within several degrees of due west, $400 \mathrm{ft}$ horizontally into the southeast slope of Fran Ridge at an altitude of $3409 \mathrm{ft}$. The purpose of the hole was to obtain data pertinent for radionuclide transport studies in the Topopah Spring Member of the Paintbrush Tuff. This unit had been selected previously as the host rock for the potential underground nuclear waste redository at Yucca Mountain, adjacent to the southwestern part of the Nevada Test Site.

The hole was core drilled first with air, then with air mist, and finally with air, soap, and water. Many problems were encountered, including sloughing of tuff into the uncased hole, vibration of the drill rods, high rates of bit wear, and lost circulation of drilling fluids. On the basis of experience gained in drilling this hole, ways to improve horizontal coring with air are suggested in this report.

All of the recov red core, except those pieces that were wrapped and waxed, was examined for lithophysal content, for fractures, and for fracture-fill mineralization. The results of this examination are given in this report. Core recovery greater than $80 \%$ at between 209 and $388 \mathrm{ft}$ permitied a fracture frequency analysis. The results are similar to the fracture frequencies observed in densely welded nonl ithophysal tuff from holes USW GU-3 and USW G-4. The fractures in core from UE-25h\#1 were found to be smooth and nonmineralized or coated with calcite, silica, or manganese oxide. Open fractures with caliche (porous, nonsparry calc: te) were not observed beyond $83.5 \mathrm{ft}$, which corresponds to an overburden depth of $30 \mathrm{ft}$.

\section{INTRODUCTION}

The Nevada Nuclear Haste Storage Investigations (NNWSI) are a U.S. Department of Energy project to determine whether a nuclear waste repository, if located in hydroiogically unsaturated tuff beneath Yucca Mountain, Nevada, would meet the safety criteria for licensing that have been established by the 
U.S. Nuclear Regulatory Commission. An important aspect for assessing the expected postclosure performance of such a repository is the retardation that radioactive species would underge relative to the flow of water that might transport them through tuff. Certain retardation oroderties, such as sorption, orecipitation, diffusion, and chemical speciation, can be measured in laboratory experiments. Computer-based models can extraculate the data from laboratory measurements to field conditions. Modeling accuracy can be confirmed by measuring radionuclide retardation under field conditions. Laboratory measurements of retardation in the presence of tuff and computerbased modeling had progressed during 1980 to the point where a field test of migration was planned. The program plan for this field test (Erdal et al. 1981) identified the Miocene Tunnel Bed 5 tuff of G-Tunnel, at the Nevada Test Site, is the location for a fracture-flow experiment under vadose zone conditions. This field test was not performed for three reasons. First, the bedding planes in the tuff proved to be no more permeable to water flow than was the tuff matrix. Second, sorotion measurements were much less reproducible with Tunnel Bed 5 tuff than with other tuffs. Finally, the modeling calculations indicated that water was unlikely to flow through a fracture in this tuff in the manner initially expected. A redort by Norris et al. (1982) documents the laboratory and modeling results from the Tunnel Bed 5 tuff work.

The search for a suitable location in which to perform a radionuclide migration field test in tuff shifted in 1982 from G-Tunnel to Yucca Mountain. By this time the Topopah Soring Member of the Paintbrush Tuff had been chosen as the Yucca Mounta. n target horizon (DOE 1984). If Yucca Mountain was chosen by the President as one of the three potentially acceptable nuclear waste redository sites, site characterization activities would include construction of an Exploratory Shaft for in situ testing. In 1982 the Exploratory Shaft construction was not scheduled to commence until 1984, and it would take about two years longer to excavate completely the main underground facility in the Topopah Spring Member. Our thinking was that a facility in the Topopah Spring Member that was not Dart of the Exploratory Shaft would be useful during the interim to investigate fracture flow characteristics in this tuff and problems associated with conducting a field test. Consequently, an exposure of Topopah Spring Member was located on the southeast flank of Fran Ridge that might be suitable for construction of an adit in which this field test could be performen (Fig. 1). The lithostatic load would not be equal to that of the 


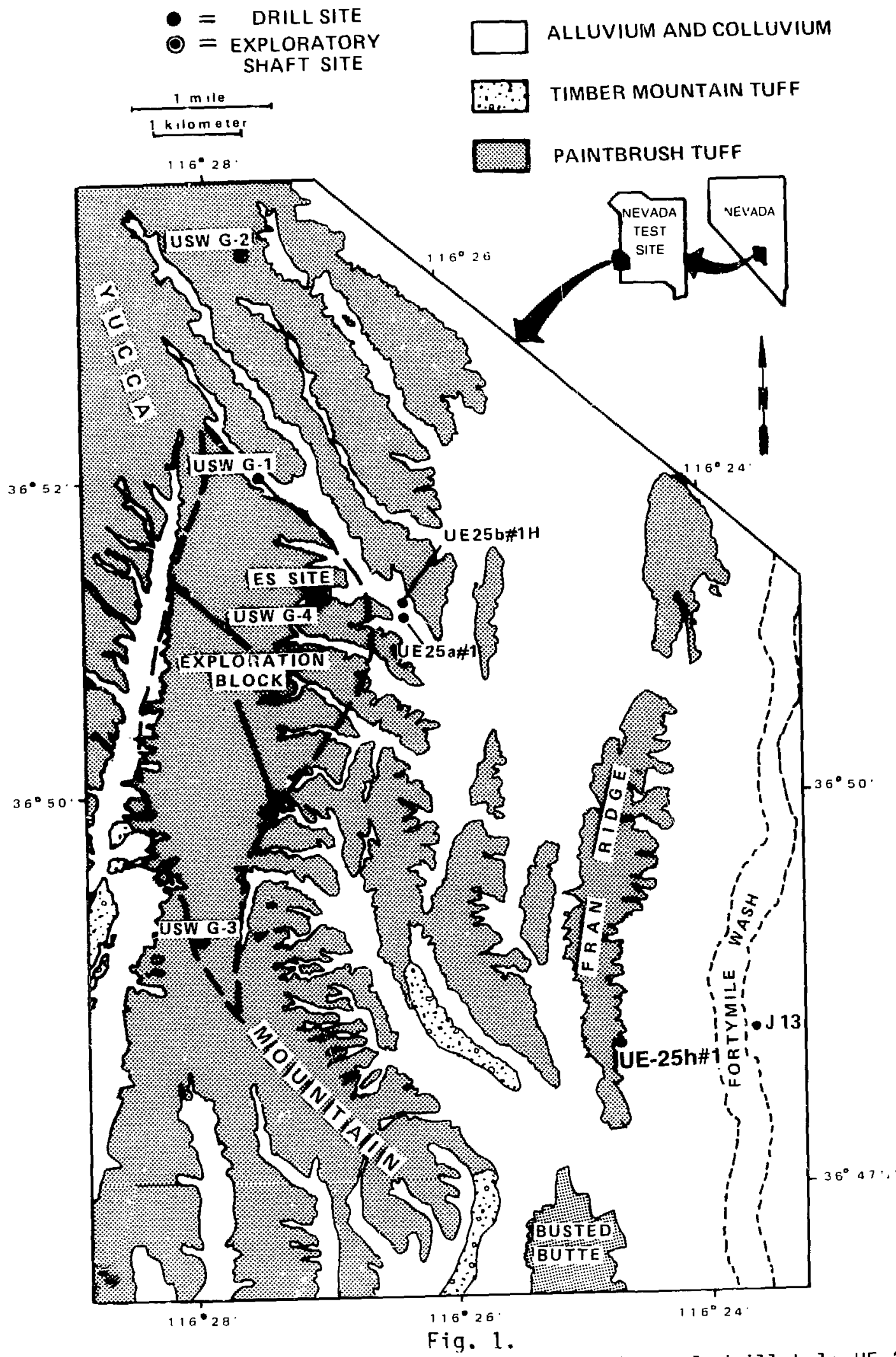

Map of Yucca Mountain area, showing location of horizontal drill hole UE-25h\#1. 
Exploratory Shaft underqround facility, but this difference wouli not impede development of the field test procedures.

Two 4-in.-diameter holes were to be cored horizontally to a distance of $400 \mathrm{ft}$ as the first part of the work to characterize the suitability of the Fran Ridge site for an adit. The horizontal distance was determirad by the maximum horizontal penetration of the adit. A deeper adit would require mechanical ventilation, and this complication was not desirable. One of the two 4-in.-diameter holes was to be located about $10 \mathrm{ft}$ above the other, and both holes were to be cored with air to avoid introducing water into the formation. After coring, isolated segments of the two holes would be tested to determine the extent to which water would flow between them. The cores would be examined to determine the fracture patterns in this tuff.

In actuality only one hole was drilled. The location of this hole, designated UE-25h\#l, is shown in Fig. 1. The Nevada State Coordinates of the hole are $N .748,353.08 \mathrm{ft}, E .574,461.38 \mathrm{ft}$, and the elevation, 3,409.38 ft ( $\mathrm{L}$. Price, $y$ imes \& Narver, Inc., written commun., June 2, 1986). The geologic mapping of Scott and Bonk (1984) indicates that the stratigraphic thickness of the Topopah Spring Member at the location of UE-25h\#1 is approximately 1000 $\mathrm{ft}$, which is comparable to the thickness penetrated in USW G-4. The subunit cored by UE-25h\#l corresponds to the middle nonlithophysal zone observed in USW G-4 core in the depth interval from 680 to $770 \mathrm{ft}$. This subunit was identified by observing an upper lithophysal zone above a contact some $30 \mathrm{ft}$ upslope from the UE-25h\#l drill collar. The upper lithophysal zone is a distinct subunit characterized by spheroidal lithophysae averaging about $5 \mathrm{~cm}$ in diameter. The lower lithophysal zone, which underlies the subunit in which UE-25h\#l was drilled, is exposed southward up the component dip in a cutbank of the dry wash that truncates the south end of Fran Ridge. At the time of drilling, the Exploratory Shaft breakout zone was expected to be the lower nonlithophysal zone. The middle and lower nonlithophysal zones of the Topopah Spring Member are mineralogically and petrographically similar (Byers 1985). The physical properties of the subunit in which UE-25h\#1 was drilled were expected to be nearly identical to those in the subunit that would contain the main underground facility of the Exploratory Shaft.

Funding from the NNWSI geochemistry program was provided to cover the estimated costs of the two core holes originally planned at Fran Ridge. 
However, IJE-?5h\#1 cost twice as much as estimated. The presumption that the start of Exoloratory Shaft construction was imminent resulted in no further funding for an in situ geochemical test facility at. Fran Ridge.

This redort oresents a history of the UE-25h\#1 drilling operations and the results of a Detrographic analysis of the core that was recovered. Section II is a summary of the drilling operations. Some readers may find a detailed drilling history useful because horizontal coring in highly fractured Topopah Spring Member tuff is not a routine operation. Therefore, a log of daily activities is included as Appendix $A$. We have listed in Section III our recommendations for future horizontal coring in this tuff so that others can profit from our experience. The core analysis results are given in Section IV. Aopendix $B$ contains the detailed log of the core examination. The data for the fracture frequency analysis in the interval from 209 to $388 \mathrm{ft}$ are tabulated in Adpendix $C$.

\section{HOLE HISTORY}

A summary of the UE-25h\#l drilling operations is presented in this section. Details are listed in Appendix A. The hole was drilled by employees of the Reynolds Electrical and Engineering Company (REECo). Fenix \& Scisson, Incorporated (F\&S) provided drilling specialists and geologists during the coring of this hole. Los Alamos National Laboratory personnel observed most of the drilling operations.

Site uredaration commenced the first week of December 1982. Bruce $M$. Crowe, Los Alamos, specified the precise hole location. Drilling to core the first $20 \mathrm{ft}$ started on December 10. A water-cooled 6.25-in. bit was used to permit emblacement of casing in cement. The casing served as a collar to help reduce drill-string vibrations during subsequent air-coring operations. Water circulation was lost at $17 \mathrm{ft}$, but cementing and casing solved the problem.

The next 165-ft interval of UE-25h\#l was cored with air, and 3.937-in. bits were used. The coring proceeded very slowly. Eight shifts were required to drill this interval, but three of these shifts were devoted to developing technology to overcome some of the problems that were encountered. The problems included high rates of bit wear, drill-string vibrations that caused slow drilling and malfunctioning of the core retrieval system, loss of air circulation, and sloughing of the tuff, which apparently caused the breakage of one drill bit. Four other bits were worn out in drilling this interval. 
Core recovery was $73 \%$, but $65 \%$ of the recovered core was broken. Work ceased for the Christmas holidays on December 17 .

The drilling oroblems, the slow drilling rate, and the expense involved in coring the first $185 \mathrm{ft}$ of UE-25h\#l clearly showed that it would be impossible to drill two air-cored holes at this site with the funds available. Therefore, the objective was changed to obtaining unbroken core to a depth of $400 \mathrm{ft}$ in one hole as expeditiously as possible. When coring recommenced on January 3, 1983, the first lubricant used was an air and water mist. This lubricant proved ineffective in solving the drill-string vibration and bit wear problems. The lubricant was changed to air, soad, and water. The water supply rate was 10 qal. Der minute. Lithium chloride was added to the water as a tracer. Drilling from $185 \mathrm{ft}$ to $216 \mathrm{ft}$ required seven shifts. Two bits were worn out. Core recovery was 52\%, and all recovered core was broken. Work was suspended on January 5 while the drilling crews were employed at USW G-4.

Drilling commenced again at UE-25h\#1 on January 18. Severe sloughing had occurred. Nine shifts were required to clean out the hole to $216 \mathrm{ft}$. The hole was drilled, rather than cored, to $220 \mathrm{ft}$. The next $17-\mathrm{ft}$ interval was cored, but with lost circulation of the lubricating fluid. The drilling engineers decided that the best way to complete this hole was to line the Dortion already comoleted and reduce the size of the drill bits to $3.032 \mathrm{in}$. for the remainder of the coring.

Coring from 240 to $400 \mathrm{ft}$ occurred during eight shifts. Four bits were used, of which three were worn out. Core recovery was much better than in the previous drilling, most likely because the $240 \mathrm{ft}$ of lining seemed to dampen the drill-string vibrations.

The completed hole was logged with a Birdwell 3-arm calider. Electronic noise from this tool compromised the logging record to the extent that fracture patterns of $J E-25 \mathrm{h \# l}$ were attempted with a Westech television camera, which could not Denetrate beyond an obstruction in the casing at $94 \mathrm{ft}$.

Hole UE-25h\#l was started horizontally on a due west heading. Surveys of heading and inclination taken during the course of drilling were used to construct the diagrams of the hole that are shown in Fig. 2 .

A total of 68 drilling crew shifts were used to core this $400-\mathrm{ft}$ hole. The volume of water and soap used to core from $185 \mathrm{ft}$, where air coring 

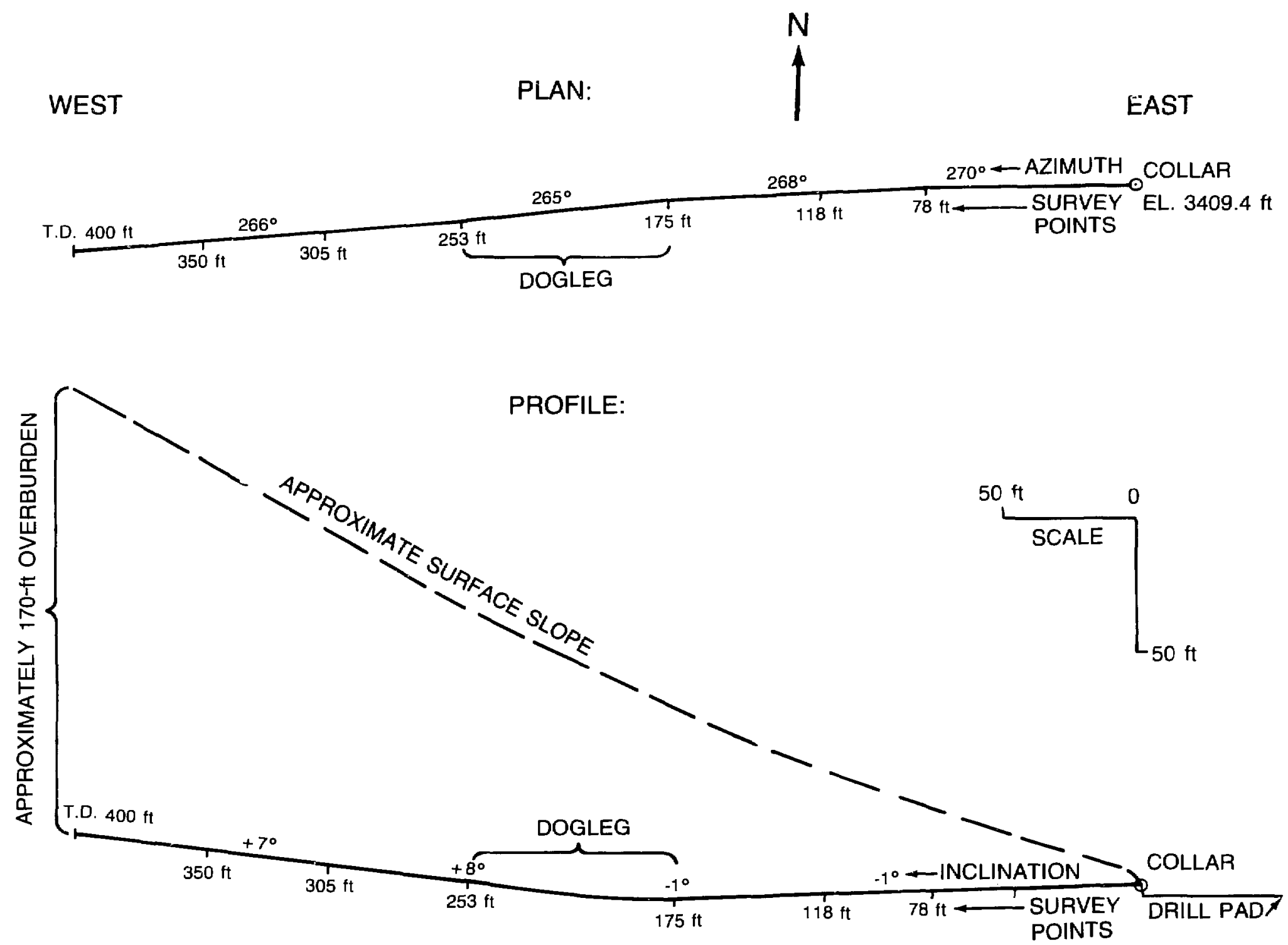

Fig. 2.

Plan and profile of horizontal drill hole UE-25h\#1. 
ceased, was 1,438 bbls $(60,396$ gal. $)$. Poor returns resulted in most of this water remaining in the formation.

III. DRILLING TECHNOLOGY: OBSERVATIONS AND RECOMMENDATIONS

Our objective in drilling at Fran Ridqe was to obtain data pertinent for radionuclide transport studies in the welded tuff of the Topopah Spring Member. Developing air-drilling technology was also part of the plan. The nonroutine nature of this horizontal coring promots us to offer the following observations to held those who may require horizontal air coring in the Exploratory Shaft.

(1) In fractured rock, horizuntal holes tend to be less stable than vertical holes because the gravity loading of rock fragments is normal to the hole axis in horizontal holes.

(2) Air drilling does not apdear to cool the drill bits adequately.

(3) Vibration of the drill string, caused by inadequate drilling fluid lubrication, contributed to Door core recovery and aggravated sloughing of material into the hole.

(4) Orill tool design was not ootimized for cooling with air, and probably several bits were damaged when they were withdrawn through the unstable hole. Perhaps a reaming cutter should be tried to aid tool withdrawal.

(5) During early drilling, vibration of the drill string compromised the integrity of the hole and may have compounded problems such as lack of drilling fluid returns, nontrue drilling directions, and standing water in the finished hole. Better drilling results might have been obtained, once a decision was made to use water as a lubricant, if a new hole had been started.

(6) The rock was harder than the drillers had expected. Perhaps more experience is needed to determine the proper combination of thrust, bit revolutions per minute, and air pressure and volume to minimize vibration and maximize bit 1 ife.

The following possibilities should be considered in future horizontal airdrilling demonstratiors.

(1) Using a bit that provides large coolant passages and a chamfer or reaming cutter to protect the bit during tool removal. 
(2) Recording accurate real-time data on bit thrust, revolutions per minute, air pressure and volume, and extent of vibration. These data will permit optimization of drilling parameters.

(3) Developing horizontal drilling in a prototypical lithostatic stress tield with prototypical rock strength and fracture characteristics.

(4) Not allowing an uncased horizontal hole to remain without drill string or casing for longer than several hours.

\section{CORE ANALYSIS RESULTS}

Each piece of recovered core from the $400 \mathrm{ft}$ of drilling, except for those pieces that were wrapped and waxed, was examined for fractures, for fracture-fill mineralization, and for lithophysal content. The number of fractures and their orientations were important in assessing the potential utility of this Topopah Spring Member exposure for field measurements of radionuclide transoort by aqueous flow in fractures. Knowledge of the fracture-fill mineralization is necessary for calculating radionuclide sordion characteristics under aqueous flow conditions and for determining the depth of surface water effects. The lithophysal content of the core is nearly zero; only a few small lenticular lithophysae, $0.5 \times 2.0 \mathrm{~cm}$, were observed.

Figure 3 (in pocket) is a graphic log of structural features of the core. Because the core orientation was not recorded, all single fractures are arbitrarily shown dioping west in Fig. 3. With intersecting fractures, the one that makes the higher angle with the horizontal core axis is arbitrarily shown dipping west.

Two fracture frequency figures are shown in Fig. 3 for the horizontal depth interval between 209 and $388 \mathrm{ft}$. The fraction of core recovered in this interval was sufficiently high, slightly greater than $80 \%$, to justify this analysis. The fractures per linear meter are shown as dots connected with dashed lines. The original data are tabulated in Appendix $B$. The mean number of fractures per linear meter is 22 . The linear fracture frequency is converted to a hypothetical set of fractures normal to the core axis for a unit cubic meter sphere (Scott et a1. 1983). This conversion is performed to permit a combarison of the UE-25h\#l data with similar U.S. Geological Survey (USGS) data already available from vertical holes on Yucca Mountain. Fractures per unit cubic meter, $F_{C}$, are calculated through application of the formula (Scott et al. 1983) 


$$
F_{c}=(\cos A)^{-1} \cdot F_{m} \text {, }
$$

where $A$ is the measured acute angle between the vertical plane perpendicular to the core axis and the fracture set, and $F_{m}$ is the observed linear fracture frequency of the fracture set. For a preselected footage interval, usually the core run of $10 \mathrm{ft}$, the products of the preceding formula are simmad over the footage interval, multiplied by the oialleter $(4.07 \mathrm{ft})$ of the unit meter sphere, and divided by the footage interval. A computer program was written and executed to perform these operations ( see Appendix C).

The calculated fractures per unit cubic meter for the UE-25h\#l data are plotted as histograms for the 209- to 388-fi interval in Fig. 3 . The mean number of fractures per unit cubic meter is 59 for the Fran Ridge core. The USW GU-3 data in Scott and Castellanos (1984) give an average of 42 fractures per unit cubic meter for the moderately to densely welded tuff of the Topopah Spring Member. The Fran Ridge data for the same lithology of the Topopah Soring Member corroborate the USW GU-3 data concerning the highly fractured character of the Topopah Spring Member. Whether this formation is too highly fractured to permit the isolation of a single fracture for radionuclide transport studies (which was the reason for this coring) is a question that can be answered only by hydiraulic conductivity measurements between two boreholes. The absence of a second borehole at UE-25h\#l precludes the possibility of such measurements.

The tuff and the mineralization that were observed in the core recovered from UE-25h\#l can be characterized by the following generalizations. The tuff resulted from a welded ash flow. It is devitrified, hard (Mohs scale $H$ 7), and densely welded. The densities of four pieces from the final $25 \mathrm{ft}$ of coring are $2.21,2.23,2.21$, and $2.21 \mathrm{~g} / \mathrm{cm}^{3}$. The intergranular porosity of the tuff is near zero.

The minerals filling the fractures are calcium carbonate, manganese oxides, and silica minerals. The location of each fill material is indicated by a colored mark on the core map, Fig. 3. Breccia fill in fractures at 17 $\mathrm{ft}, 131 \mathrm{ft}$, and $155 \mathrm{ft}$ indicates that the fractures may be part of a fault system. Calcium carbonate deposits fill fractures within certain intervals throughout the $400 \mathrm{ft}$ of core. However, calcium carbonate in the form of 
caliche was not observed beyond $83 \mathrm{ft}$, which appears to be the limit for surface water effects. The ground surface has a slope of $20^{\circ}$ at UE-25h\#1 (Fig. 2). Therefore, the $83-\mathrm{ft}$ horizontal distance is about $30 \mathrm{ft}$ beneath the ground surface. A sample of calcium carbonate fill from a fracture at $285 \mathrm{ft}$ was analyzed by $x$-ray diffraction techniques. The fill is 90 to $100 \%$ calcite and about $1 \%$ quartz (Ellen Semarge, Sandia National Laboratories, written commun., 1983). Materials filling other fractures are nanganese oxides and silica minerals. The silica fill in a fracture at $266 \mathrm{ft}$ was determined. using $x$-ray diffraction techniques, to contain 85 to $95 \%$ tridymite and 10 to 15\% quartz (Ellen Semarge, Sandia National Laboratories, written commun., 1983).

The tuff contains light- and dark-brown lenticular pumice inclusions throughout the nole, ranging from less than $1 \mathrm{~cm}$ to $5 \mathrm{~cm}$ in diameter. Generally, the pumice is flattened; the flat plane of the inclusion is nearly parallel to the axis of the core. The only indication of lithophysae anywhere in the core occurs in a few pumice inclusions located bciween 266.5 and 272 $\mathrm{ft}$. The lithophysal cavities can best be described as incipient, although they do contain minute vapor phase crystals. Nevertheless, the general character of the cored Topopah Spring Member is lithophysal free, like the Exploratory Shaft target unit (DOE 1984). However, the Topopah Spring Member at Fran Ridge is not the stratigraphic equivalent of the target unit: the lithophysal-free zone at Fran Ridge is interbedded between lithophysae-bearing strata, whereas the target unit, as penetrated in drill hole USW G-4, underlies all the lithophysal zones (Spengler and Chornack 1984). The essentially nonlithophysal, devitrified subunit tested at Fran Ridge most likely correlates with a similar subunit penetrated in USW G-4 from 680 to $770 \mathrm{ft}$ beiween two lithophysal zones (Spengler and Chornack 1984). The lithologic character of this zone and that of the target unit, however, are essentially the same.

\section{ACKNOWLEDGMENTS}

R. B. Scott of the USGS provided helpful advice on measuring fractures in the core and on distinguishing natural fractures from driling-induced fractures. He also identified the UE-25h\#l target horizon as the middle nonlitliophysal zone of the Todopah Spring Member, based on his geologic mapping of Fran Ridge (Scott and Bonk 1984). 
The work of the following Los Alamos National Laboratory personnel also is acknowledged. S. D. Francis provided technical advice. J. P. Hong and $S$. W. White wrote a computer program to calculate the fractures per cubic meter. B. E. Hahn typed the manuscriot, and P. L. Aamodt contributed helpful criticism that improved the text.

\section{REFERENCES}

Byers, F. M., Jr., "Petrochemical Variation of Topopah Sprinq Tuff Matrix with Depth (Stratigraphic Level), Drill Hole USW G-4, Yucca Mountain, Nevada," LoS Alamos National Laboratory report LA-10551-MS (1985).

DOE (U.S. Department of Energy), "Draft Environmental Assessment, Yucca Mountain Site, Nevada Research and Development Area, Nevada," DOE/RW-0012, Washington, D.C., DD. 2-43 (1984).

Erdal, B. R., K. Wol fsberg, J. K. Johnstone, K. L. Erickson, A. M. Friedman, S. Fried, and J. J. Hines, "Nuclide Migration Field Experiments," Los Alamos National Laboratory redort LA-8487-MS (1981).

Norris, A. E., R. D. Aguilar, B. P. Bayhurst, D. L. Bish, M. R. Cisneros, W. R. Daniels, C. J. Duffy, R. D. Golding, S. L. Jensen, S. D. Knight, F. 0 . Lawrence, S. Maestas, A. J. Mitchel1, P. Q. 01 iver, N. A. Raybold, R. S. Rundberg, G. M. Thompson, E. N. Treher, B. J. Travis, G. R. Walter, R. G. Warren, and $K$. Wolfsberg, "Geochemistry Studies Pertaining to the G-Tunnel Radionuclide Migration Field Experiment," Los Alamos Vational National Laboratory report LA-9332-MS (1982).

Scott, R. B., R. W. Spengler, S. Diehl, A. R. Lappin, and M. P. Chornack, "Geologic Character of Tuffs in the Unsaturated Zone at Yucca Mountain, Southern Nevada," in Role of the Unsaturated Zone in Radioactive and Hazardous Waste Disoosal, $\sqrt{\text {. W. Mercer, P. S. C. Rao, and I. W. Marine }}$ TEds.), Ann Arbor Science, Ann Arbor, Michigan, 289-335 (1983).

Scott, R. B. nd M. Castellanos, "Preliminary Report on the Geologic Character o Drill Holes USW GU-3 and USW G-3," U.S. Geol. Surv. Open-File rieport JSGS-OFR-B4-491 (1984).

Scott, R. B., and J. Bonk, "Preliminary Geologic Map of Yucca Mountain, Nye Courty, Nevada, with Geologic Sections," U.S. Geol. Surv. Open-File Report USGS-OFR-84-494 (1984).

Spengler, R. W., and M. P. Chornack, "Stratigraphic and Structural Characteristics of Volcanic Rocks in Core Hole USW G-4, Yucca Mountain, Nye County, Nevada," with a section on "Geophysical Logs," by D. C. Muller and J. E. Kibler, U.S. Geol. Surv. Odan-File Rept. USGS-OFR-84-789 (1984). 
APPENDIX A

UE-25h\#1 HOLE HISTORY--DAILY REPORT

(Compiled by J. C. McDaniel, drilling superintendent, Reynolds Electrical and Engineering Company, with minor additions from the Fenix and Scisson drill specialist's daily report.).

12-09-82 Rigged uD Acker drill, rigged up 900 compressor. No approval to drill yet.

12-10-82 16 hours rigging up.

3:30 PM - Received OK to start drilling.

Cored 6-1/4-in. hole from 0 to $16 \mathrm{ft}-100 \%$ recovery $-2 \mathrm{~h}$, using clear water with Bean Dumb. 150 rpm, 2000\# on bit. No pressure.

Full returns.

12-11-82 Cored 6-1/4-in. hole from 16 to $20 \mathrm{ft}-1 \mathrm{~h}$.

(Note) Lost circulation at $17 \mathrm{ft}$.

Ran 4-1/2-in. H.W. casing to $20 \mathrm{ft}$.

Cemented same with $17 \mathrm{cu}$. ft $\mathrm{Cal}$ Seal. Good cement returns.

Cored 3.937-in. hole from 0 to $20 \mathrm{ft}-8 \mathrm{ft}$ recovery. Coring with air only, 900 compressor.

Cored from 20 to $30 \mathrm{ft}, 10 \mathrm{ft}-1 \mathrm{~h}, 10 \mathrm{ft}$ recovered.

$100 \mathrm{rpm}, 2000 \#$ on bit, 10 stabilized core harrel.

Lost crown off bit.

12-i2-82 Coring 3.937-in. hole, air only, $900 \mathrm{cfm}$.

30 to $62 \mathrm{ft}-40 \mathrm{rpm}, 2000 \#$ on bit, $150 \mathrm{psi}$, lots of vibration on rods - breaking ears off latch-in head.

At $68 \mathrm{ft}$, picked up $15-\mathrm{ft}$ core barrel.

(Note) Bits \#3 \& \#4 - lost part of crown.

Short runs, really fractured, poor recovery.

12-13-82 Coring 3.937-in. hole, air only, $900 \mathrm{cfm}$.

92 to $126 \mathrm{ft}-34 \mathrm{ft}$ in $10 \mathrm{~h}$.

30 to $40 \mathrm{rpm}, 2000^{\#}$ on bit, 160 psi, lots of vibration.

Survey at $118 \mathrm{ft}-89^{\circ}, 2^{\circ} \mathrm{S} . \mathrm{W}$. (See summary on hole deviation at end).

12-15-82 Coring 3.937-in. hole, air only, $900 \mathrm{cfm}$, days only.

12.6 to $144 \mathrm{ft}$ - $18 \mathrm{ft}$ with $15-\mathrm{ft}$ core barrel.

Christensen on location - 2 men. 
Lots of vibration of rods.

Christensen trying to make pump-in tool work and rew air bits.

12-16-82 3.937-in. hole, air only, $900 \mathrm{~cm}$.

144 to $182 \mathrm{ft}$ - $38 \mathrm{ft}$, lots of yibration, it is breaking the latch-head spririgs.

Two Christensen men on location working on Dimp-in tools and air bits.

(Note) $167 \mathrm{ft}$ - lost air returns.

Pressure dropped from 225 psi to 50 psi, lots of sloughing prohlems from 45 to $182 \mathrm{ft}$. Cleaning hole in and out.

Survey at $175 \mathrm{ft}-89^{\circ}, 2^{\circ} \mathrm{S} . \mathrm{W}$. (See summary at end).

(Note) from 20 to $167 \mathrm{ft}$, hole dusting well.

12-17-82 3.937-in. hole, air only, $900 \mathrm{cfm}$.

182 to $185 \mathrm{ft}-3 \mathrm{ft}$.

Cleaning out with 3-3/4-in. tri-cone bit, no returns, hole won't clean up.

10:00 AM - Trioped in with core barrel, cleaned fill from 162 to $182 \mathrm{ft}$.

11:30 AM - Cored from 182 to $185 \mathrm{ft}, 3 \mathrm{ft}-1 \mathrm{~h}, 1 / 2 \mathrm{ft}$ recovered.

Lrts of torque and vibration.

Christensen men on location - 2 men.

2:00 PM - Secured operation for Christmas Holidays.

(Note) Acker drill ram, 0-ring leaking, will have to be replaced before continuing coring or cleaning out.

01-03-83 3.937-in. hole, air and mist, lithium chloride.

Ted Norris, LANL, gave OK for air and mist, then, air and soap.

Total water pumped in $=46 \mathrm{bbls}$ or $1932 \mathrm{gal}$. fluid.

8:00 AM - Started up, replaced packing in Acker drill, rigged up fluid tank and pump.

4:00 PM - Tripped in with 3-3/4-in. rock bit, reamed from 160 to $185 \mathrm{ft}$ with air and mist, no returns, 10ts of vibration.

$6 \mathrm{~h}$ - Kept trying to clean hole, no success.

Steve Francis, LANL, gave OK to go to air and soap. 
01-04-83 3.937-in. hole, air and soap and $1 \mathrm{ithium} \mathrm{chloride,} 900 \mathrm{cfm}$.

185 to $200 \mathrm{ft}-15 \mathrm{ft}-100 \mathrm{rpm}, 1000 \#$ on bit, $50 \mathrm{psi}$, fluid $10 \mathrm{gpm}$. Cleaning out with 3-3/4-in. rock bit.

6:00 AM - Tripped in hole with core barrel, fill at $170 \mathrm{ft}$, cleaned out with core barrel.

9:00 AM - Cored from 185 to $188 \mathrm{ft}-3 \mathrm{ft}$ - $1 \mathrm{~h}$ - blocked.

Re iurnea inner barre?, recovered one core - fractured.

Pump-in tool seemed to be working well. Cored from 185 to $200 \mathrm{ft}$, $15 \mathrm{ft}$.

(Note) Vibration is getting tetter, $100 \mathrm{rpm}, 2000 \#$ on bit, 220 psi, Bean 35 - $5 \mathrm{gpm}$ fluid, 80\% recovery - fractured.

0:-05-83 Coring 3.937-irr. hole, air and soap and lithium chloride, $900 \mathrm{~cm}$. 200 to $216 \mathrm{ft}-16 \mathrm{ft}-105 \mathrm{rpm}, 1000$ on bit, $150 \mathrm{psi}, 10 \mathrm{gpm}$ soap. (Note) $209 \mathrm{ft}$ - tripped out, laid down 15-ft Christensen core barrel, picked up 10-ft Longyear core barrel. $209 \mathrm{ft}$ - trip - fluid back in $80 \mathrm{ft}$.

$216 \mathrm{ft}$ - trip out - fluid back in $180 \mathrm{ft}$.

Longyear core barrel and pump-in equipment work better than Christensen - had $50 \mathrm{ft}$ to clean out with Longyear barrel, small amount of vibration at 130 to $150 \mathrm{rpm}$.

(Note) 2:30 PM - Secured oderation to go to USW G-4, will have meeting about 01-13-83 with LANL, F\&S, DOE, and REECo about the completion of these holes.

Total days - 9-2/3 days.

01-18-83 Moved equipment from USW G-4 to UE-25h\#1. 3.937-in. hole, $216 \mathrm{ft}$, air and soap and lithium chloride. Rigged up.

Tripped in hole with Christensen 3.937 Geoset bit, shell, 10-ft Longyear barrel. Fill at $73 \mathrm{ft}$, cleaning out to $193 \mathrm{ft}$ with air and soap, no returns, 200 psi.

3:30 PM: Tube plusged, tnols stuck. Retrieved inner barrel, pumped in 35 gal. water with \#3 popper, air and soap, worked tools out from 193 to $184 \mathrm{ft}$, tight. Came free.

5:00 PM: nut of hole, left Geoset bit and shell and threads of 
A) PDENDIX A (cont)

core barrel in hole. Fish at $184 \mathrm{ft}$. Working Acker head about $40,000 \#$ push and Dull.

10:00 PM: Tripped in hole with tap on 3-1/2-in. $x 2-3 / 8-i n$. IF drill pipe. Pushed fish tn $192.65 \mathrm{ft}$, tripped out, no fish.

01-19-83 Tripped in hole with 1.9 tubing open ended. Cleaned fill from: 155 to $185.62 \mathrm{ft}$. Good returns, no pressure. 1.9 stopped at 185.62 ft. Tripped in with tap on IF drill pipe, worked tap in from 185 to $197 \mathrm{ft}$. Tripped out, no fish.

10:00 AM: Tripped in with tap, 3-1/2-in. 0.0. $x$ 1-1/4-in. I.D., $191 \mathrm{ft} \mathrm{fill,} \mathrm{worked} \mathrm{tap} \mathrm{in} \mathrm{to} 193 \mathrm{ft}$, tapped $91 \mathrm{ug}, 700 \mathrm{psi}$, worked tap loose, tripped out, left tap in hole, 3-1/2-in. 0.0. $x$ 1-i/4-in. I.D. - $1 \mathrm{ft}$ long.

12:30 PM: Tripped in with taper tap $3 / 4$ to $2 \mathrm{in}$. to $163 \mathrm{ft}$ fill, plugged tap. Tripped out, unplugged, tripped in, fill at $155 \mathrm{ft}$, cleaned out from 155 to $196 \mathrm{ft}, 200 \mathrm{psi}$, no returns. Tripped in with taper tap on 2-3/8-in. If drill pipe, pushed fish to 212.48 ft. Tripped out, no fish. Tripped back in with taper tap to $195.18 \mathrm{ft}$.

01-20-83 Cleaned from 195.18 to $197 \mathrm{ft}$, no returns, tools olugged, tripped out. Changed out 900 compressor for HHE 1200. Tripped back in with tap. Reamed from 151 to $160 \mathrm{ft}$, started getting returns with LCM 20\# with water, won't drill anymore, olugged. Tripped in with 1.9 tubing open ended to $150 \mathrm{ft}$, pumped in LCM. Tripped sut, tripped in with 3-3/4-in. rock bit, cleaned fill from 159 to $216 . i 0$ ft. Tripped out, cones gone off bit, partial returns.

01-21-83 3.937-in. hole, $216 \mathrm{ft}$, air and soap and lithium chloride. From 216 to $237 \mathrm{ft}-21 \mathrm{ft}$ in $4 \mathrm{~h}$. Lost $7 \mathrm{ft}$ core, good rock.

1:00 AM: Tripped in hole with 1.9-in. tubing to $180 \mathrm{ft}$, mixed heavy soap. Pumped in from 160 to $180 \mathrm{ft}$, conditioned hole, good returns. 200 psi, 1 - 1200 compressor, Bean 35 pump.

5:00 AM: Tripped in hole with HQ core barrel, reained from 150 to $199 \mathrm{ft}$, stopped. 
9:00 AM: Tripped out. Drill tools out to $150 \mathrm{ft}$, partial returns, pumped in $17 \mathrm{gpm}, 5$-gpm returns, no air returns, $150 \mathrm{psi}$.

10:30 AM: Trioped in with 3-3/4-in. flat bottom mill to $196 \mathrm{ft}$, cleaned out to $220 \mathrm{ft}$, no returns, air or soap. Tripped back in with core barrel, washid from 140 to $220 \mathrm{ft}$ using air, soap, and lithium chloride, partial returns, air - soap, 300 psi.

3:00 PM: Coring from 220 to $237 \mathrm{ft}$, 1 ittle returns. (Note) Lest 7 $\mathrm{ft}$, good core.

4:00 PM: Talked to Ted Norris and Hammer abcut reducing hole size to 3.032 in., OK'ed by Ted Norris, LANL.

10:30 PM: Inner barrel stuck, tripped tools.

01-22-83 3.937-in. hole, $237 \mathrm{ft.}$.

1:00 AM: Tripped in hole 3-1/2-in. HCQ rods, with reaming shoe to $184 \mathrm{ft}$, washed and reamed into $216 \mathrm{ft}$, stopped.

9:30 AM: Tripped out, tight hole, reamed out, very little returns, air/soad, casing shoe worn out.

11:30 AM: Made up casing shoe, 3-3/4 in., tripped in, fill at 196 to $218 \mathrm{ft}$, stopped. Tripped out. Shoe worn out. Very little returns, no air returns. Made up 3-3/4-in. flat bottom mill, tripped in with 2-3/8-in. IF drill pipe.

4:00 PM: Reamed and washed from 196 to $235.5 \mathrm{ft}$, stopped. Little returns.

9:00 PM: Tripped out, mill worn out. Tripped back in with 3-3/4-in. flat bottom mill to $217 \mathrm{ft}$.

01-23-83 3-3/4-in. hole to $240 \mathrm{ft}$. Reamed with flat bottom mill from 217 to $240 \mathrm{ft}$, made $3 \mathrm{ft}$ of new hole, no returns, Bean 35, no air.

4:00 AM: Tripped out with 2-3/8-in. IF drill pipe, tripped in with 3-1/2-in. HCQ rod with reaming shoe.

5:00 AM: Reamed from 158 to $170 \mathrm{ft}$, soap onily, no returns, $170 \mathrm{ft}$ stopper.

6:00 AM: Tripped out, left reaming shoe 13-1/2-in. 0.D. $x 6$ in. long) + 2.5-ft HCQ rod in hole. (Note): Total junk left in hole at this time - depth 160 to $190 \mathrm{ft}$ : 3.937-in. core bit, 6-in.-1ong reaming she11, with threads of core barrel pulled off, taper tap - 


\section{APPENDIX A (cont)}

2-1/4 to 3-1/4 in., 1-1/4-in. I.D., $1 \mathrm{ft}$ long. Three cones off 3-3/4-in. bit. Recovered 2 cones, 3-1/2-in. casing shoe 6 in. long, 2/5-ft $\mathrm{HCQ}$ dril1 rod 3-1/16-in. I.D.

8:00 AM: Trioped in hole with 3-1/16-in. Casing spear on 2-3/8 IF drill pioe, cleaned out fill from 151 to $171 \mathrm{ft}, 1-1 / 2 \mathrm{~h}$, no returns, air and soap, $100 \mathrm{psi}$. Tripped out, no fish, mandrel on spear was bent due to pushing in.

11:30 AM: Tripped back in with taper tap, 2-1/4 in. $\times 3-1 / 2$ in. :-1/4-in. I.D., $1 \mathrm{ft}$ long - new. Fill at 160 to $167 \mathrm{ft}$, tap plugged. Tripped out, unplugged tap, tripped in with same tap, washed from 153 to $167 \mathrm{ft}$, can't get past $167 \mathrm{ft}$. Tap completely worn out.

2:00 PM: Tripped in with 3-3/4-in. Flat bottom mill on 2-3/8 IF drill Dipe, reamed from 150.5 to $172.20 \mathrm{ft}-2 \mathrm{~h}$, mill worn $1 / 2 \mathrm{in}$. - not too bad.

5:30 PM: Tripped in with 1.9-in. tubing open ended to $172 \mathrm{ft}$, tried to wash past $172 \mathrm{ft}$ with air and soap, 200 psi, pumping in 17 gom, returns $5 \mathrm{gpm}$, very little air, tripped out.

6:30 PM: Tripped in with 3-1/4-in. casing spear on 2-3/8-in. IF drill pipe to $172.70 \mathrm{ft}$, tripped out, no fish.

8:00 PM: Tripped in with 3.937 impregnated bit on HCQ rod, reamed from 161 to $181 \mathrm{ft}$, no returns, tripped out. $1-1 / 2 \mathrm{ft}$ of rocks in rods, bit worn out.

01-24-83 3-3/4-in. hole, $240 \mathrm{ft}$. Tripped in with wash shoe on 3-1/2-in. HCO rod, cleaned out fill from 136 to $161 \mathrm{ft}$, good returns, air and soap, $60 \mathrm{psi}$. Tools good and free, reamed to $191 \mathrm{ft}$, reamed and rereamed 170 to $191 \mathrm{ft}$, partial returns, $40 \mathrm{psi}$, air and soap.

9:30 AM: Tripped out, shoe worn out.

10:30 AM: Tripped in with 3-3/4-in. flat bottom mill on 2-3/8-in. IF drill pipe. Reamed from 150 to $240 \mathrm{ft}-5 \mathrm{~h}$. Reamed and rereamed, partial returns, 100 psi, conditioned hole.

3:00 PM: Tripped out mill, not uad. 
4:00 PM: Tripped in with casing shoe on 3-1/2-in. HCQ rod. Reamed from 150 to $194.30 \mathrm{ft}-4-1 / 2 \mathrm{~h}$, stopped. Had $1 \mathrm{ft}$ partial returns, rocks in $\mathrm{HCQ}$ rod.

10:30 PM: Built up flat bottom mill. 3-3/4 in. - $24 \mathrm{~h}$ total fluid used: 156 bbls. Total fluid pumped in hole: 479 bbls.

01-25-83 3-3/4-in. hole to $240 \mathrm{ft}$. Reamed with 3-3/4-in. flat bottom mill from 130 to $240 \mathrm{ft}$, reamed and rereamed. Water and lithium chloride only, $30 \mathrm{rpm}, 0 / 100 \mathrm{psi}, 35 \mathrm{gpm}$ - no returns. Total fluid pumped in this $24 \mathrm{~h}-355$ bbls.

1:00 PM: Tripped out, mill not bad.

2:00 PM: Tripped in with HCQ rod, homemade shoe, reamed in from. 190 to $240 \mathrm{ft}$, dry landed $\mathrm{HCQ}$ at $240 \mathrm{ft}$.

3:00 PM: Tripped in with 3-in. homemade mill shoe with 2-3/4-in. NCQ drill rod, cleaned out $\mathrm{HCQ}$ rod from 170 to $240 \mathrm{ft}$ with water only.

10:30 PM: Trisped out to check mill - OK. Tripped back in - $1 \mathrm{~h}$ milled on 3-1/2-in. casing shoe - $240 \mathrm{ft}-40 \mathrm{rpm}$, water only, 20 gpm, 20 psi.

01-26-83 3-1/2-in. HCQ set at $240 \mathrm{ft}, 2 \mathrm{~h}$ milling on HCQ casing shoe, 50\% returns. Tripped out mill - 1/16 in. under gauge.

3:00 PM: Tripped in with 3.032 core barrel and bit, milled through shoe at $240 \mathrm{ft}$.

3:30 PM: Coring from 240 to $272 \mathrm{ft}$ - 32-ft short runs, blocking off every 4 or $5 \mathrm{ft}, 200 \mathrm{rpm}, 2 / 4000$ \# on bit, $200 \mathrm{psi}, 2 \mathrm{gpm}-1$ compressor, $95 \%$ core recovery. Survey at $253 \mathrm{ft}-98^{\circ} 5 \mathrm{ft} \mathrm{S-W}$. Total fluid used this $24 \mathrm{~h}-60 \mathrm{bbls}$.

01-27-83 3-1/2-in. HCQ set at $240 \mathrm{ft}$. Coring 3.032 hole from 272 to $333 \mathrm{ft}$ - $61 \mathrm{ft}$ - 85\% core recovery, short runs, blocking off. $200 \mathrm{rpm}$, 2/4000\# on bit, 200 psi, 20 gpm - 1 compressor, 25 to $50 \%$ fluid returns, very little air. Survey at $305 \mathrm{ft}-97^{\circ} 40^{\prime} \mathrm{S}-\mathrm{W}$.

4:00 PM: (Note): $316 \mathrm{ft}$, hole getting very tight. No returns, tripped out, bit inner gauge worn, tripoed in with new bit.

6:00 PM: Washed from 316 to $318 \mathrm{ft}$. 
APPENDIX A (cont)

8:00 PM: Coring, very little returns. Total fluid used this $24 \mathrm{~h}$ - 108 bbls.

01-28-83 3-1/2-in. HCQ rod set at $240 \mathrm{ft} .3 .032$ nole from 333 to $388 \mathrm{ft}$ $55 \mathrm{ft}, 90 \%$ core recoverv. $150 \mathrm{rpm}, 4000$ \# on bit, $150 \mathrm{psi}, 20 \mathrm{gpm}-$ 1 compressor. itile fluid returns.

1:30 $\mathrm{fiv}: 333 \mathrm{ft}$, tried to pull inner barrel, broke ears on tube, tripped rods, inner barrel crushed, trying to rig up another inner barrel.

8:00 AM: Received inner barrel from area 12, made up new Longyear assembly, tripped in hole, no fill.

2:00 PM: Coring 333 to $388 \mathrm{ft}$. Total fluid used this $24 \mathrm{~h}-121$ bbls.

01-29-83 3-1/2-in. HCQ ros set at $240 \mathrm{ft}, 3.032$ hole, 388 to $400 \mathrm{ft}-2 \mathrm{~h}-$ $12 \mathrm{ft}-105 \mathrm{rom}, 2000 \#$ on bit, $100 \mathrm{psi}, 20 \mathrm{gpm}, 1$ compressor.

2:00 AM: TO at $400 \mathrm{ft}$ - no returns. Tripped out, tight hole, $355-\mathrm{ft}$ hole very tight, working stuck pipe from 355 to $345 \mathrm{ft}$ with Bean 35 pump only.

11:00 AM: Tripped inside NCQ rod with 1.9-in. tubing with taper tad, $1-1 / 2$ in. $x$ 2-1/4 in., to retrieve inner barrel, core inside, can't work tap in inner barrel. Rigged up $5 \times 8$ pump, working stuck pipe 3 it free, working dipe hard.

6:00 PM: Rigged up air, mixed 20 gal. soap, pumped in working pipe.

9:00 PM: Pipe rotating, working pipe free, tripped out laying down tools. Total fluid used this $24 \mathrm{~h}-154 \mathrm{bbls}$.

01-30-83 3-1/2-in. HCQ rods set at $240 \mathrm{ft}, 3.032$ hole at $400 \mathrm{ft}$. Moved out equipment to rig up Birdwell.

3:00 AM: Ran Birdwell calider log, pushed in on 1/2-in. aluminum rods, stopped at $290 \mathrm{ft}$, loqged from 290 out to $240 \mathrm{ft}$. Checked tools, reran same, stopped at $290 \mathrm{ft}$, tripped caliper.

5:00 AM: Tripped in with NCQ rod with 3-in. mill, cleaned out with mill from 198 to $380 \mathrm{ft}$ with Bean pump, $35 \mathrm{gpm}$, water only, hole getting tight, rigged up $5 \times 8$ pumb, pumping to $60 \mathrm{gpm}$, cleaning hole to $400 \mathrm{ft}$ - no returns, conditioned hole. 
12:30 PM: Reran calider 109, stopped at $293 \mathrm{ft}$. Rigged down Birdwel1, ran Westech TV camera, 2-7/8-in. 0.D., stopped at $94 \mathrm{ft}$, at $45 \mathrm{ft}$ camera started in dirty water.

4:00 PM: Rigged down equipment and moved to UE-25b\#1. Total fluid used in this $24 \mathrm{~h}-161 \mathrm{bbls}$. Total fluid pumped in UE-25h\#l 1438 bbls of water, soap, lithium chloride solution.

Total days - 22-2/3 operating days.

(Notes): Tools left in hole: 3-1/2-in. HCQ rods, $240 \mathrm{ft}$ with reaming shoe, dogleg 175 to $253 \mathrm{ft}-68 \mathrm{ft}$.

Junk left in hole: 3.937 bit 5 in. long, reaming shell, $t$-in. taper tap 2-1/4 in. $\times 3-1 / 4$ in. $\times 1-1 / 4-i n$. I.D., 2 cones of 3-3/4in. bit, reaming shell 6 in., 2-1/2-ft HCQ rod

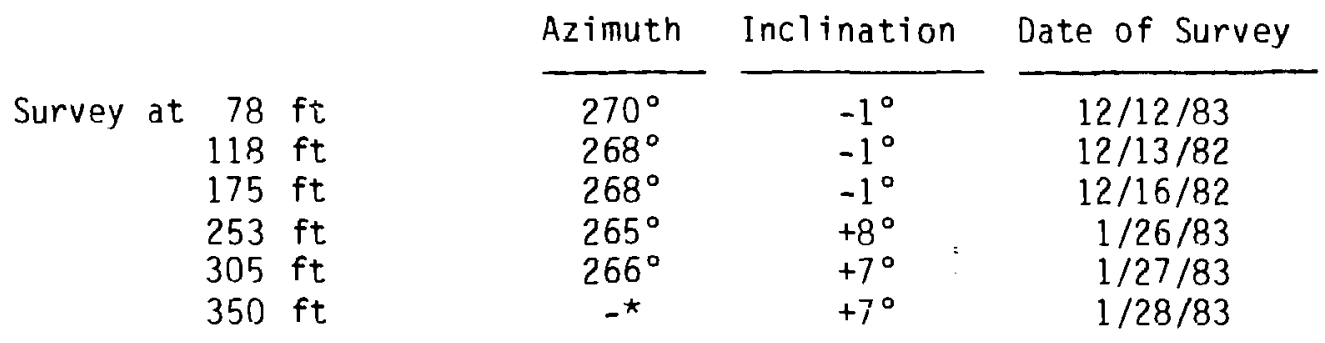

*Unable to read direction. 


\title{
APPENDIX A (cont)
}

DRILL BIT RECORD

\author{
$\# 1$ - New Christensen 6-1/4 in. - 0 to $20 \mathrm{ft}, 2-1 / 2 \mathrm{~h}$ - Good \\ $20 \%$ recovery. \\ \#2 - New Christensen $3.937 \mathrm{in.}$ - 20 to $30 \mathrm{ft}-1 \mathrm{~h}$ - \\ left crown in hole $100 \mathrm{rpm}, 2000 \#$ on bit, air \\ only, 50 psi. Surface set.
}

\#3 - Used Huddy Impregnated $3.937 \mathrm{in}$ - 30 to $46 \mathrm{ft}$ - Worn out

$16 \mathrm{ft}-2 \mathrm{~h}$. $1 / 3$ of crown left in hole.

$60 \mathrm{rpm}, 75 \mathrm{psi}$, air only, $2000 \#$ on bit.

\#4 - New

Longyear Impregnated - 46 to $68 \mathrm{ft}$ -

Worn out

$22 \mathrm{ft}-3 \mathrm{~h}, 60 \mathrm{rpm}, 3000$ \# 0 bit, $150 \mathrm{psi}$,

air only, lots of vibration.

$\# 5$ - New Christensen Chrisdriil - carbide inserts

68 to $78 \mathrm{ft}$ - $10 \mathrm{ft}$ - $1 \mathrm{~h}$ - inserts

nissing, $1 / 3$ of crown gone - pulled off.

\#6 - Used Truco Impregnated - 78 to $111 \mathrm{ft}$ - $33 \mathrm{ft}$ - Worn out

$6 \mathrm{~h}, 40 \mathrm{rpm}, 2000 \#$ on bit, $150 \mathrm{psi}, 900 \mathrm{~cm}$.

$\# 7$ - New

Geoset, Christensen - 111 to $185 \mathrm{ft}$ -

Worn out

$73 \mathrm{ft}-4 \mathrm{~h}, 40 \mathrm{rpm}, 2000 / 4000 \#$ on bit,

air only, no returns at $167 \mathrm{ft}$.

Christensen on location.

\#8- New

3-3/4-in. rock bit, clean out $185 \mathrm{ft}$ -

Fair

went to air and mist.

\#9 - New

Christensen Impregnates $=185$ to $209 \mathrm{ft}$

Worn out

$24 \mathrm{ft}-3 \mathrm{~h}, 100 \mathrm{rpm}, 2000 \#$ on bit,

220 psi, air and soâp.

$\# 10-$ New

Wesdrill S.S. - 209 to $216 \mathrm{ft}-7 \mathrm{ft}$ -

Worn out

$1-1 / 2 \mathrm{~h}, 130 \mathrm{rom}, 6000 \#$ on bit,

150 psi, $10 \mathrm{gpm}$.

Total cost - New bits at $\$ 1,400.00$ each - $\$ 11,200.00$

(Note) Bits 11 through 14 were homemade REECo flat bottom mills and casing shoes, no cost.

\#15 - New Wesdrill from 240 to $253 \mathrm{ft}-13 \mathrm{ft}-3 \mathrm{~h}$, Worli out $200 \mathrm{rpm}, 4000 \#, 200 \mathrm{psi}, 20 \mathrm{gpm}, 1200 \mathrm{c} \mathrm{fm}$, $25 \%$ fluid returns.

\#16 - New Wesdrill $3.032,253$ to $316 \mathrm{ft}-63 \mathrm{ft}-14 \mathrm{~h}$ Inside gauge worn $200 \mathrm{rpm}, 2 / 4000 \#$ an bit, $200 \mathrm{Dsi}, 20 \mathrm{gpm}$, $1200 \mathrm{cfm}, 25$ to $50 \%$ fluid returns. 


\section{APPENDIX A (cont)}

$\begin{array}{lll}\# 17 \text { - New } & \text { J. K. Smith } 3.032,316 \text { to } 333 \mathrm{ft}-17 \text { it }- & \begin{array}{l}\text { Worn from } \\ \text { cleaning out }\end{array} \\ & 2 \mathrm{~h} & \\ \text { \#18 - New } & \text { Longyear } 3.032,333 \text { to } 400 \mathrm{ft}-67 \mathrm{ft}, & \text { Good } \\ & 12 \mathrm{~h}, 150 \mathrm{rpm}, 2000 \# \text { on bit, } 200 \mathrm{psi}, & \\ & 20 \mathrm{gpm}, 1200 \mathrm{cfm} .\end{array}$

Total cost of bits and core barrels - $\$ 14,000.00$. 
APPENDIX 6

\section{DETAILED FRACTURE LOG OF HORIZONTAL DRILL. HOLE UE-25h\# 1}

The zone consists of generally grayish-red, dense, hard, moderately to densely welded tuff; rare light-gray rhyolite xenoliths, $1 \mathrm{~cm}$ in maximum dimension; rare-to-sparse flattened pumice, maximum $2 \mathrm{~cm}$ in length, with vapor phase crystals of cristobalite/tridymite $\left(\mathrm{SiO}_{2}\right)$ and alkali feldspar. Caliche (porous $\mathrm{CaCO}_{3}$ ) and calcite (sparry $\mathrm{CaCO}_{3}$ ) identified with dilute $\mathrm{HCl}$. Silica minerals (quartiz and tridymite) identified by $E$. Semarge, using x-ray diffraction. Thicknesses of mineral codtings on fractures less than $0.5 \mathrm{~mm}$ were not measured. Lithologic and fracture log by F. M. Byers, Jr.

\section{Description of Footage Interval (angles are measured from core axis)}

Ash-flow tuff, densely welded, complex stockwork of fractures filled with caliche; in middle of interval, $5-\mathrm{cm}$ caliche vein cuts core at $10^{\circ}$.

Ash-flow tuff, densely welded footages and angles of fractures:
7.0: $35^{\circ} 1-\mathrm{cm}$ yeinlet caliche
7.2: $45^{\circ} 1-$ mim veinlet caliche
7.4: $60^{\circ} 1-\mathrm{mm}$ veinlet caliche
7.8: $45^{\circ} 1-\operatorname{mm}$ veinlet caliche
7.9: $30^{\circ}$ intersecting $45^{\circ}$ above
8.1: $45^{\circ} 1-\mathrm{mm}$ veinlet caliche

Ash-flow tuff, densely welded, complex stockwork of broken core along caliche-filled fractures.

As above, two pieces of core separated and broken along 3-mm caliche-filled fracture.

As above, broken core with many caliche-coated fractures.

As above, core piece truncated at $12.8 \mathrm{ft}$ by $1-\mathrm{mm}$ caliche-coated fracture, $60^{\circ}$.

As above, broken core, caliche-coated surfaces.

Ash-flow tuff, dale brown, densely welded, minor vapor phase crystals in pumice; core in $0.3-$ to 0.8 - ft Dieces; core broken near $90^{\circ}$, mostly drilling induced.

$\begin{array}{cl}\begin{array}{c}\text { Horizontal } \\ \text { Footage }\end{array} & \text { Thickness of } \\ (\mathrm{m}) & \mathrm{Interval} \text { in } \\ \end{array}$

\section{$0.0-6.4$ \\ 6.4}

$(0.0-1.95)$

(1.95)

$\begin{array}{ll}6.4-8.4 & 2.0 \\ (1.95-2.56) & (0.61)\end{array}$

\begin{tabular}{|c|c|}
\hline $\begin{array}{c}8.4-10.2 \\
(2.56-3.11)\end{array}$ & $\begin{array}{l}1.8 \\
(0.55)\end{array}$ \\
\hline $\begin{array}{l}10.2-11.0 \\
(3.11-3.35)\end{array}$ & $\begin{array}{c}0.8 \\
(0.24)\end{array}$ \\
\hline $\begin{array}{l}11.0-12.0 \\
(3.35-3.66)\end{array}$ & $\begin{array}{l}1.0 \\
(0.31)\end{array}$ \\
\hline $\begin{array}{l}12.0-12.8 \\
(3.66-3.90)\end{array}$ & $\begin{array}{c}0.8 \\
(0.24)\end{array}$ \\
\hline $\begin{array}{l}12.8-13.6 \\
(3.90-4.15)\end{array}$ & $\begin{array}{l}0.8 \\
(0.24)\end{array}$ \\
\hline $\begin{array}{l}13.6-17.4 \\
(4.15-5.30)\end{array}$ & $\begin{array}{l}3.6 \\
(1.10)\end{array}$ \\
\hline
\end{tabular}


APPENDIX B (cont)

Description of Footage Interval (angles are measured from core axis)
Horizontal

Footage

(m)
Thickness of

Interval in

$\mathrm{ft}(\mathrm{m})$

Fractures at footages:

16.8: $40^{\circ}$ 2-cm-wide fault breccia

17.1: $40^{\circ}$ fracture intersects fault breccia at end of core.

Ash-flow tuff, as preceding interval, broken core, cal iche-coated surfaces.

$17.4-18.0$

$(5.30-5.48)$

$18.0-20.0$

$(5.48-6.10)$
0.6

(0.18)

2.0

$$
\begin{aligned}
& \text { As preceding, less broken core, fractures at } \\
& \text { following footages: } \\
& \text { 18.0: } 60^{\circ} \text { caliche coated } \\
& \text { 18.2: } 30^{\circ} \text { " } \\
& \text { 18.6: } 30^{\circ} \text { clean, possibly drilling induced } \\
& \text { 19.0: } 70^{\circ} \text { rough, purplish alteration } \\
& \text { 19.1: } 45^{\circ} \text { microbreccia, } 5 \mathrm{~mm} \text { thick } \\
& \text { 19.2: } 90^{\circ} \pm \text { possibl ". } 117 \text { ing induced } \\
& \text { 19.5: } 60^{\circ} \text { smooth } \\
& \text { 19.6: } 60^{\circ} \text { smooth in rsects above } \\
& \text { 20.0: core bi kf irregularly }
\end{aligned}
$$

As preceding, about $50 \%$ welded tuff breccia fragments in cement grout used to anchor casing.

Ash-flow tuff, core variably broken from small pieces, granule-size to one 0.9-in.-length core from 22.0 to $22.9 \mathrm{ft}$. Most core fragments caliche coated and bounded by irregular surfaces. Fractures at following footages:

24.5: $45^{\circ}$

24.7: $45^{\circ}$ intersects above

25.3: $30^{\circ}$ planar, no caliche

Ash-flow tuff, as preceding, variably broken core from small pebble-size pieces to $0.5-\mathrm{ft}$-maximum length. Three waxed intervals $32.0-32.4 \mathrm{ft}, 32.55-$ $33.05 \mathrm{ft}$, and 33.75-34.1 ft. Broken core surfaces irregular, Dossibly drilling induced. Dccasional caliche-coated surface.

Ash-flow tuff, as preceding, variably broken with intermittent swarms of pebble-size fragments alternating with larger pieces of core up to $0.6 \mathrm{ft}$. Waxed intervals at 49.4-49.65, 50.2-50.5, 58.5-58.9, 60.0-60.25, and 59.9-70.45 ft. Thin ( $\pm 1 \mathrm{~mm})$ caliche coated. Surfaces at following footages:

51.0: $45^{\circ}$

$59.0: 40^{\circ}$

$$
\begin{array}{ll}
25.3-47.4 & 22.1 \\
(7.71-14.45) & (6.74)
\end{array}
$$

$\begin{array}{lc}20.0-20.6 & 0.6 \\ (6.10-6.28) & \\ 20.6-25.3 & 4.7 \\ (6.28-7.71) & (1.43)\end{array}$ $\begin{array}{cc}47.4-83.5 & 36.1 \\ (14.45-25.45) & (11.00)\end{array}$ 
Description of Footage Interval (angles are measured from core axis)

$\begin{array}{cl}\text { Horizontal } & \text { Thickness of } \\ \text { Footage } & \text { Interval in } \\ (\mathrm{m}) & \mathrm{ft}(\mathrm{m})\end{array}$

$83.5-100.0$

16.5

$(24.45-30.48)$

\section{essentially zero intergranular or matrix Dorosity. Core is mostly broken along hackly irregular breaks, probably largely drilling induced. Fracture footage, angle with core axis, description: \\ 83.5: $45^{\circ} 4-\mathrm{mm}-$ thick caliche veinlet \\ 85.0: $0^{\circ} \pm$ a few $\mathrm{cm}$ long, curved, with thin Mn oxide coating}

Ash-flow tuff with common small pumice lenticl
less than $1 \mathrm{~cm}$ length. Hardness $6-1 / 2$ to 7 ,

\section{3: $50^{\circ}$ Dlanar}

87.4-87.9: $5-15^{\circ}$ curved, smooth

89.6-90.7: $5^{\circ}$ fracture in central part of several core pieces, Mn oxide coating overlain by 1-mm-thick sporadic coating of sparry calcite $\left(\mathrm{CaCO}_{3}\right)$

91.3-91.8: $10-20^{\circ}$ rough fracture with very thin $(<0.1-\mathrm{mm})$ coating of sparry calcite

92.3-92.7: $30^{\circ}$ rough fracture with thin calcite (from here to end of hole, calcite is sparry)

94.0-94.2: $3^{\circ}$ rough

95.4-95.9: $0^{\circ} \pm<0.1-\mathrm{mm}$ coating of calcite

98.7-99.0: $30^{\circ} \quad 0.1 \pm-\operatorname{mm} \mathrm{SiO}_{2}$ minerals

99.7: ? chip, smooth, planar, Mn oxide coated.

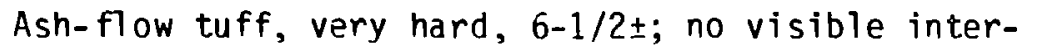
granular porosity. Core mostly broken into chips with only several pieces 0.2-0.4 ft long.

Fracture footages, angle, and description:

104.6-105.0: $0^{\circ} \pm$ slightly curved, white gougy material

106.7-107.6: $20^{\circ}$ gouge, calcareous

$\begin{array}{ll}\text { 108.6: } & 45^{\circ} \text { planar, smooth } \\ \text { 110.8: } & 45^{\circ} \text { planar, Mn oxides } \\ 111.2: & 30^{\circ} \text { " }\end{array}$

Ash-flow tuff, as above; less breakage than previous run with 4 core pieces 0.4 to $0.7 \mathrm{ft}$. Waxed core, 113.6-114.0. Fracture footages, angle, and description:

112.6-112.3: $0^{\circ} \pm$ rough

113.2:

$35^{\circ}$ planar
$100.0-111.6 \quad 11.6$

$(30.48-34.02) \quad(3.54)$ $\begin{array}{lc}111.6-114.6 & 3.0 \\ (34.02-34.93) & (0.91)\end{array}$ 
APPENDIX B (cont)

Description of Footage Interval (angles are measured from core axis)

\begin{tabular}{cl} 
Horizontal & Thickness of \\
Footage & Inter:al in \\
$(\mathrm{m})$ & $\mathrm{ft}(\mathrm{m})$ \\
\hline
\end{tabular}

$114.6-120.0$

$(34.93-36.58)$ footages:

117.2-117.5: planar surface with 1-nm coating of calcite

119.0-119.6: a few chips with planar surface with $M n$ oxide coating $<0.1 \mathrm{~mm}$.

Ash-flow tuff, as preceding, hard, no visible intergranular porosity. Pieces of core 0.2 to $0.5 \mathrm{ft}$ long al ternate with broken core, $0.2-$ to 0.5-ft intervals. Intervals broken core from 126-137 ft contain subrounded pebble-size chips, probably drilling induced and recovery is about $50 \%$. Elsewhere in this run, core recovery is $85 \%$ or better. No visible pumice, very fine grained, with sparse phenocrysts, lithic granules, and $M n$ oxide specks. Fractures at following footages:

120.3: $45^{\circ}$ planar, <0.1-mm Mn oxide coating

(120.3-120.7): waxed core

122士: $\quad 80^{\circ}$ planar, Mn oxide coated

(123.1-123.5): waxed core

131 $\pm: \quad 45^{\circ}$ rough, calcite-cemented micro-

136士: $\quad 10^{\circ}$ rough, $<0.5-\mathrm{mm}$ calcite

136.5: $\quad 60^{\circ}$ smooth, <0.1-mm Mn oxide

136.6: $\quad 10^{\circ}$ rough, <0.5- $\mathrm{mm}$ calcite (intersects 136.5 at $30^{\circ}$ )

137.5: ? smooth, <0.05-mm Mn oxide

143.5: $20^{\circ}$ smooth, Mn oxide dendrites

144.0: $\quad 65^{\circ}$ smooth, planar

144.7: $\quad 40^{\circ}$ rough, planar

144.8: $45^{\circ} "$ " "

148.2-148,4: $0^{\circ}$ planar, smooth, <0.5-mm calcite coating

150.7: $\quad 45^{\circ}$ rough, planar

151.1: ? smooth, planar.

Ash-flow tuff, as preceding. Core broken, subangular to subrounded, except for 3 pieces, 0.2-0.3 ft. Densely welded with flattened pumice lenticles, $0.5-3.0 \mathrm{~cm}$, making $10-25^{\circ}$ foliation attitude with core axis; probably

$\begin{array}{ll}120.0-152 \pm & 32.0 \pm \\ (36.58-46.3 \pm) & (9.75 \pm)\end{array}$
$152.0 \pm-153.5 \quad 1.5 \pm$ $(46.33 \pm-46.79) \quad(0.45 \pm)$ 
Description of Footage Interval (angles are measured from core axis)

$\begin{array}{cl}\text { Horizontal } & \text { Thickness of } \\ \text { Footage } & \text { Interval in } \\ (\mathrm{m}) & \mathrm{ft}(\mathrm{m})\end{array}$

represents east dip; fractures at following footages:
$152 \pm$ :
$60^{\circ}$ rough
152.5:
$65^{\circ}$ smooth, planar, Mn oxide coating.

Ash-flow tuff, hard, dense, core broken, subangular. Maximum dip of foliation, $15^{\circ}$. Four core pieces 0.3-0.4 in. long. Fractures and angle with core axis at following footages:

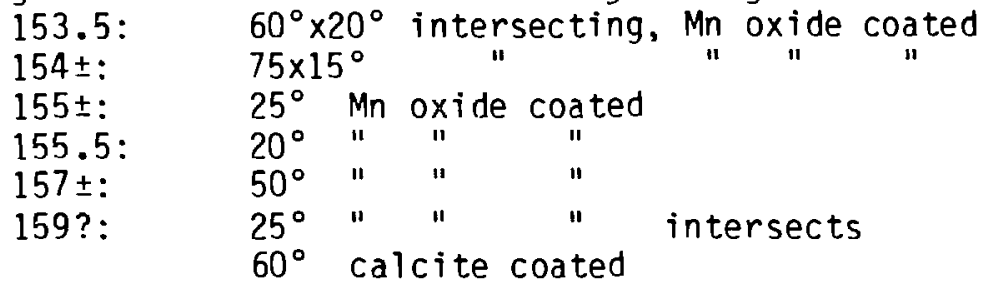

$153.5-159 \pm$

$(46.79-48.46 \pm)$
$5.5 \pm$ $(1.67 \pm)$

Core broken and mostly missing, recovery less than $50 \%$

Ash-flow tuff, as preceding, except maximum dip (angle with core axis but probably indicating

$159.0 \pm-163.0 \quad 4.0 \pm$ $(48.46 \pm-49.68) \quad(1.22 \pm)$

$163.0-173.0 \quad 10.0$ $(49.68-52.73) \quad(3.05)$ easterly dipl is $20^{\circ}$. Core broken with 4 pieces, 0.2 to $0.3 \mathrm{ft}$ long. Fractures at following estimated footages:

163-164: At least 2 Mri-oxide-stained fractures

164.5: $\quad 55^{\circ}$ calcite-coated fracture intersects $35^{\circ}$ calcite-cemented 2 -mm-thick fault microbreccia

134.5-173.0: broken core, less than 50\% recovery. About $6 \mathrm{Mn}$-oxide-coated fractures of undeterminable orientation.

Ash-flow tuff, as preceding, core broken into pebble-size chips with two pieces 0.3-0.4 ft long. Fractures at following footages:
173.5:
calcite, 0.5-m coating irregular surface
$174 \pm:$ three planar fractures on broken
$175 \pm:$ surfaces
$175.5:$
176.0:
$10^{\circ} \pm$ Mn-oxide-coated fracture
$176.5:$
$10^{\circ}$ one Dlanar fracture
$176.6:$
calcite ud to $2 \mathrm{~mm}$ thick on broken end of 0.4-ft core piece. 


\section{Descriotion of Footage Interval (angles are measured from core axis)}

Ash-flow tuff, as preceding, no visible pumice; broken core. At least 2 dianar fractures of undeterminable orientation.

Ash-flow tuff, as preceding, but run laboled "Fill from undetermined depth"... Core rounded and beveled by drilling.

As preceding, recovered $0.2 \mathrm{ft}$

(Changed from air to air mist and soap--see Appendix A)

Ash-flow tuff, densely welded, hard, pumice $<1 \mathrm{~cm}$ length, $20^{\circ}$, assumed to dip easterly as in surface outcrop. Core almost entirely broken with rotated pieces grooved by drill bit at varying angles. Breakage aopears drilling induced.

Ash-flow tuff, as preceding, about $30 \%$ recovery.

Ash-flow tuff, as preceding. Core broken with 3 Dieces 0.3 to $0.4 \mathrm{ft}$ long. Core recovery $66 \%$. Fractures at footages and angle with core axis:

191.5: $45^{\circ}$ Dumice foliation is $5^{\circ}$

191.8: $45^{\circ}$ Dumice foliation, $0^{\circ} \pm$

192.2: $55^{\circ} 0.5 \mathrm{~mm}$ calcite coating

192.4: $60^{\circ}$ Dlanar

193.2: $60^{\circ}$

193.6: $55^{\circ}$ Mn oxide stained

193.8: $60^{\circ}$

Ash-flow tuff, broken, three 0.4-ft Dieces, Dumice $<2 \mathrm{~cm}$ long, $<5^{\circ}$ from core axis, $87 \%$

core recovery. Fractures and angles with core axis:

194.5: $45^{\circ}$ olanar

194.8: $35^{\circ}$

$195.2: 45^{\circ}$

195.5: 2 intersecting at $90^{\circ}$

196.1): $45^{\circ}$ and $10^{\circ}$

196.5: $80^{\circ}$ Dianar, Mn oxide coating

$197.5: 10^{\circ}$

$197.7: 10^{\circ}$

198.0: $10^{\circ}$

198.8: $60^{\circ}$

199.2: $40^{\circ}$

199.5: $40^{\circ}$

$\begin{array}{cl}\text { Horizontal } & \text { Thickness of } \\ \text { Footage } & \text { Interval in } \\ (\mathrm{m}) & \mathrm{ft}(\mathrm{m})\end{array}$

$177.0-177.4 \quad 0.4$

$(53.95-54.07) \quad(0.12)$

$177.4-182.0 \quad 4.6$

$(54.07-55.47) \quad(1.40)$

$182.0-185.0 \quad 3.0$

$(55.47-56.39) \quad(0.92)$

$185.0-188.0 \quad 3.0$

$(56.39-57.30) \quad(0.91)$ $\begin{array}{lc}188.0-191.0 & 3.0 \\ (57.30-58.22) & (0.92) \\ & \\ 191.0-194.0 & 3.0 \\ (58.22-59.13) & (0.91)\end{array}$
$194.0-200.0 \quad 6.0$

$(59.13-60.96) \quad(1.83)$ 
Description of Footage Interval (angles are measured from core axis)

Ash-flow tuff, as preceding. Rock completely broken from sand to cobble size. At least 3 fractures of indeterminable orientation. $20 \%$ core recovery, but see below.

As preceding, Dumice foliation angle $20^{\circ}$. Recoverea $3 \mathrm{ft}$ of core, including $2 \mathrm{ft}$ from preceding run. Core largely broken along fractures, resulting in angular pieces, pebble to cobble size. Fractures include following:

No. planar

No. Mn oxide stained

No. thin calcite coating

As preceding, except for $0.3 \mathrm{ft}$ at 208.7-209.0, 87\% core recovery. Fractures include following: No. planar 7

No. Mn oxide stained (mostiy $<40^{\circ}$ from core axis) No. calcite coated

\begin{tabular}{l}
$\begin{array}{c}\text { Horizontal } \\
\text { Footage } \\
(\mathrm{m})\end{array}$ \\
\hline
\end{tabular}

200.0-204.0

(60.96-62.18)

4.0

$204.0-205.0$

(62.18-62.48) Interval in ft $(\mathrm{m})$

ckness of

Ash-flow tuff, as preceding, $100 \%$ core recovery, mostly broken except at following footages (no down arrows)

209.0-209.3: 1/2 core cut longitudinally by $15^{\circ}$ planar, ends cut by $75^{\circ} \mathrm{Mn}$ oxide coated and $60^{\circ}$ planar.

209.4-209.7: bounded by $60^{\circ}$ Mn oxide coated and $70^{\circ}$ planar, fracture within core $35^{\circ}$ planar.

209.7-209.9: bounded by $40^{\circ}$ End $65^{\circ}$ planar fractures.

209.9-210.6: broken core, following fractures: $\begin{array}{ll}\text { No. planar } & 4 \\ \text { No. Mn oxide coated } & 5\end{array}$

210.6-210.8: bounded one end, $50^{\circ}$ planar

210.9-211.3: bounded by two $45^{\circ}$ planar and one irregular $90^{\circ}$ fracture down middle of core.

211.3-211.45: bounded by subparalle1 $45^{\circ}$.

211.45-211.6: bounded on one end by intersecting $60^{\circ}$ planar and $45^{\circ}$ Mn oxide coated; al so $25^{\circ}$ Mn oxide and $60^{\circ}$ planar fractures.

211.6-211.8: 2 subparallel $40^{\circ}$ planar on each end.

211.8-212.5: Following fractures:

$\mathrm{Mn}$ oxide coated

Mn oxide coated: $40^{\circ}, 45^{\circ}, 45^{\circ}$

Planar: $45^{\circ} 45^{\circ}$ 
Description of Footage Interval (angles are measured from core axis)
Horizontal

Footage

(m)
Thickness of Interval in $\mathrm{ft}(\mathrm{m})$

212.5-212.6: Following fractures:

Planar

Mn oxide coated

212.6-213.7 ft: 3 Dieces core

212.6: $80^{\circ}$ Mn oxide coated

212.9: $40^{\circ}$ rough surface

$15^{\circ}$ Mn oxide coated

212.9-213.5: calcite coated, curved lowangle fracture. Pumice foliation, $5^{\circ}$ 213.5-213.7: $40^{\circ}, 50^{\circ}$, and $75^{\circ}$ Dlanar fractures

213.7-216.0: broken core, subangular fractures Mn oxide coated Calcite coated

Drilled with rock bit, no core

Drilled $1.0 \mathrm{ft}$; recovered $6 \mathrm{ft} ; 5 \mathrm{ft}$, sprayed with biue Daint, are "E-core," Debble-size, subangular, no fractures recognizable, may be coarse cuttings from rock bitting. 220.0-221.0 valid core, pebble to cobble size, angular to subangular. Ash-flow tuff, as preceding. Three Doorly defined fractures, $25^{\circ}, 40^{\circ}$, and indeterminate.

Ash-flow tuff, as preceding, aranule to cobble size, angular.

$\begin{array}{lc}216.0-220.0 & 4.0 \\ (65.83-67.06) & (1.23) \\ 220.0-221.0 & 1.0 \\ (67.06-67.36) & (0.30)\end{array}$

Fracture surfaces noted:

No. Dlanar, incl. one $50^{\circ} \quad 18$

No. $\mathrm{Mn}$ oxide coated, inc1. $35^{\circ}, 20^{\circ}, 5^{\circ}, 0^{\circ}$

No. $\mathrm{SiO}_{2}$ mineral coated $65^{\circ}$

Ash-flow tuff, as preceding, foliation angle $15^{\circ}$. Fractures at following footages:

224.5: $45^{\circ}$ Dlanar

224.7: $35^{\circ}$ Mn oxide stained

224.9: $0^{\circ}$ hairline, irregular

Ash-flow tuff, as preceding, pebble to cobble size, except for 1 diece,

$221.0-224.5$

$(67.36-68.43)$

$224.5-225.2$

0.7

(68.43-68.64)

$225.2-226.6$

1.4

225.2-225.5: $35^{\circ}$ planar

$40^{\circ}$ Mn oxide coated 
APPENDIX B (cont)

Description of Footage Interval

(angles are measured from core axis)

$\begin{array}{cl}\begin{array}{cl}\text { Horizontal } \\ \text { Footage }\end{array} & \begin{array}{l}\text { Thickness of } \\ \text { Interval in }\end{array} \\ \mathrm{ft}(\mathrm{m})\end{array}$

226.6-228.0

$(69.07-69.49)$

1.4

$(0.42)$

4

No. planar

5

10

No. $\mathrm{SiO}_{2}$ mineral coated 1
$26.4-226.6$ : 1 piece bounded by two parallel

planar $55^{\circ}$ fractures.

Ash-flow tuff, as preceding, broken core

following fractures

No. Mn oxide coated, incl. $60^{\circ}, 60^{\circ}, 60^{\circ}, 40^{\circ} \quad 14$

No. $\mathrm{SiO}_{2}$ mineral coated

Ash-flow tuff, as preceding, broken core

$228.0-232.0$

$(69.49-70.71)$

4.0

No. planar, $0^{\circ}, 45^{\circ}$, $15^{\circ}, 60^{\circ}, 0^{\circ}$

No. Mn oxide coated, incl.

$5^{\circ}, 45^{\circ}, 35^{\circ}, 35^{\circ}, 30^{\circ}$, $45^{\circ}, 5^{\circ}$

27

No. calcite coated

No. $\mathrm{SiO}_{2}$ mineral coated

$20^{\circ}, 0^{\circ}, 30^{\circ}, 40^{\circ}$

4

Ash-flow tuff, as preceding, $80 \%$ recovery; detail of footages:

232.0-232.3: broken core, following fractures:

$232.0-237.0$

5.0

$(70.71-72.24)$
No. Mn oxide coated 2

No. planar

232.3-232.7: 1 piece, following fractures:

$40^{\circ}$ planar

$5^{\circ}$ Dlanar

$70^{\circ}$ Mn oxide stained

232.7-233.0: core not recovered(?)

233.0-233.9: 1 piece, following fractures:

233.0: $50^{\circ} 1-\mathrm{mm}$ calcite coating

233.4: $30^{\circ}$ irregular hairline

233.9: $55^{\circ}$ Mn oxide coated

233.9-234.8: broken core, following fractures:

234.0: $55^{\circ} \mathrm{Mn}$ oxide and calcite

234.1: $0^{\circ}$ calcite coating

234.2: $65^{\circ} \mathrm{Mn}$ oxide and calcite

234.5: $75^{\circ}$ and $40^{\circ}$ planar

234.5-234.8: Mn oxide stained, 3 
APPENDIX B (cont)

Description of Footage Interval (angles are measured from core axis)
Horizontal Thickness of

Footage Interval in

(m) ft $(\mathrm{m})$

234.8-235.2: 1 piece:

234.8: $70^{\circ}$ Mn oxide coated

235.2-235.4: Assumed core loss (core below does not match with preceding)

235.4-237.0: broken core, following fractures:

235.4: $90^{\circ}$ planar

235.6-235.7: 2 parallel $65^{\circ}$

Mn oxide coated

Drilled with rock bit. Reduced diameter from

$237.0-240.0 \quad 3.0$

HCQ to NX

$(72.24-73.15)$

Ash-flow tuff, as oreceding, mostly broken,

$240.0-245.0$

5.0

except for four 0.3-0.4-ft pieces. 90\% recovery.

$(73.15-74.68)$

240.1: $40^{\circ}, 40^{\circ}$ planar

240.3: 2 planar $45^{\circ}$ intersecting

240.6: $60^{\circ}$ planar

240.6-241.0: broken core

No. planar

4

241.0-241.3: 1 piece:

$0^{\circ}$ planar

$45^{\circ}$ and $55^{\circ}$ planar

$40^{\circ} \mathrm{Mn}$ oxide stained

241.3-242.0: broken core

$0^{\circ}$ and $20^{\circ}$ planar

$45^{\circ}$ and $10^{\circ} \mathrm{Mn}$ oxide stained

242.0-242.4: 1 piece:

$70^{\circ}$ and $20^{\circ}$ hairline cracks

$45^{\circ}$ and $40^{\circ}$ planar

242.4-243.2: broken core

No. Dlanar

No. Mn oxide coated

No. $\mathrm{SiO}_{2}$ mineral coated

2

6

243.2-243.5: 1 piece; small 1-cm-long pumice

foliation, $0-5^{\circ}$ with core axis

243.2: $45^{\circ}$ planar

243.5: $70^{\circ}$ calcite coated

243.5-243.9: broken core

$10^{\circ}$ and $55^{\circ}$ planar

$45^{\circ} \mathrm{Mn}$ oxide coated

243.9-244.3:

$40^{\circ}$ rough Dlanar

244.3-244.7: broken core

$35^{\circ}, 60^{\circ}, 0^{\circ}, 0^{\circ}$

244.7-245.0: no core 
Description of Footage Interval (angles are measured from core axis)
Horizontal
Footage
(m)

Thickness of Interval in $\mathrm{ft}(\mathrm{m})$

\footnotetext{
Ash-flow tuff, moderately welded; pumice

245.0: $45^{\circ}$ planar

245.25-245.55: waxed core

245.55-245.8: $45^{\circ}, 35^{\circ}, 30^{\circ}$ hairline fractures

246.0: $45^{\circ}$ planar

246.2: $45^{\circ}$ hairline

246.3: $45^{\circ}$ rough silica mineral coated

246.3-246.5: $70^{\circ} 45^{\circ} \mathrm{Dlanar}$; $\mathrm{u}^{\circ} \mathrm{Mn}$ oxides

246.5-247.2: 1 core piece split in middle, $5^{\circ}$ :

246.5: $70^{\circ}$ planar

247.2: $75^{\circ}$ "

247.2-249.0: continuous core:

248.0: $30^{\circ}$ planar

248.1: $60^{\circ}$ "

248.2: $45^{\circ}$

248.4: $45^{\circ} \quad "$

249.0: $55^{\circ}$ and $55^{\circ}$ intersecting planars.

Ash-flow tuff, light brownish gray, pumice indistinct, not fiattened (compacted). Color change from overlying light brown gradational

$249.0-253.0$

4.0

from 240.0 to 249.0 : Core recovery, $92 \%$. Details

$(75.90-77.11)$

at following footages:

249.0-249.2: broken core

249.2: $60^{\circ}$ rough

250.1: $40^{\circ}$ rough

250.1-250.7: broken core, angular

$90^{\circ}, 45^{\circ}, 70^{\circ}$ Mn oxide coated

$90^{\circ} \mathrm{SiO}_{2}$ mineral coated

250.7: $40^{\circ} \mathrm{Mn}$ oxide coated

250.8: $5^{\circ}$ planar

251.2: $45^{\circ}$ Mn oxide coated

251.2-252.4: $5^{\circ} \mathrm{Mn}$ oxide coated

251.4: $15^{\circ}$ planar

251.6: $40^{\circ}, 30^{\circ}$, irregular, hairline

252.1: $75^{\circ}$ Mn oxide coated

252.4: $50^{\circ}$ planar, $45^{\circ}$ Mn oxide coated

252.6: $35^{\circ}$ Mn oxide coated

252.7: $45^{\circ} "$ "

252.7-253.0: broken core, angular

No. planar

No. Mn oxide coated

1

No. calcite coated

3

1
} 
Description of Footage Interval (angles are measured from core axis)

$\begin{array}{cl}\text { Horizontal } & \text { Thickness of } \\ \text { Footage } & \text { Interval in } \\ (\mathrm{m}) & \mathrm{ft}(\mathrm{m})\end{array}$

$253.0-255.0$

$(77.11-77.72)$ at following footages:
253.5: hairline crack, irregular
253.6: $45^{\circ}$ planar
253.7: $60^{\circ}$
253.9: irregular crack
254.0: $40^{\circ}$ Mn oxide coated
254.0-255.0: broken core angular
$40^{\circ}$ planar
$5^{\circ}, 45^{\circ}, 10^{\circ} \mathrm{Mn}$ oxide coated
$45^{\circ}, 65^{\circ} \mathrm{SiO}_{2}$ mineral coated
$5^{\circ}$ calcite coated.

Ash-flow tuff, as preceding, small indistinct pumice foliation makes angle near $0^{\circ}$. Details

Ash-flow tuff, core more broken than preceding run; one 0.5 piece, others $<0.2 \mathrm{ft}$, angular, $90 \%$

$255.0-259.0$

$(77.72-78.94)$

4.0
255.3: $45^{\circ}$ planar, rough

255.5: $40^{\circ}$

255.7: $35^{\circ}$ calcite coated, $45^{\circ}$ planar

255.8: $45^{\circ}$ Mn oxide coated

256.0: $35^{\circ} " 10^{\circ} \pm$ irregular, rough

256.8: $80^{\circ}$ planar

256.9-257.2: $10^{\circ} \pm$ curved, Mn oxide

$257.315^{\circ}$ Mn oxide coated

$257.545^{\circ} "$ " "

$257.775^{\circ} \pm \mathrm{Mn}$ oxide coated

257.7-257.9: $15^{\circ}$ planar, $40^{\circ} \mathrm{Mn}$ oxide, $70^{\circ}$ hairline

257.9-259.0 broken core

$15^{\circ}, 60^{\circ}$ planar

$45^{\circ}, 45^{\circ}, 55^{\circ}, 45^{\circ}$ Mn oxide coated

$20^{\circ}$ calcite coated

Ash-flow tuff, as preceding, pumice foliation near

$0^{\circ}$. Details at following footages:

259.5: $55^{\circ}$ planar

259.5-259.8: $20^{\circ} \pm$ irregular

259.8: $60^{\circ} \pm$ curved

259.8-260.4: $20^{\circ}$ hairline

260.6: $50^{\circ}$ Mn oxide coated

260.7: $45^{\circ}$ planar

261.1: $50^{\circ}$

261.1-261.7: broken, angular

261.7-261.9: $80^{\circ}$ calcite coated, $0^{\circ}$ planar

261.9-262.1: $0^{\circ}, 70^{\circ}$ olanar

262.2: $10^{\circ}$ planar
$259.0-263.5$

4.5

(78.94-80.31) 
Description of Footage Interval (angles are measured from core axis)
Horizontal

Footage

(m)
Thickness of Interval in $f t(m)$

262.3: $65^{\circ}$ Dlanar

262.4: $85^{\circ} \mathrm{SiO}_{2}$ mineral, $60^{\circ}$ planar, $45^{\circ}$ Mn oxide

262.5: $55^{\circ}$ Dlanar

262.5-262.7: broken core, angular

262.7: $70^{\circ} \mathrm{SiO}_{2}$ mineral coating

262.9: $40^{\circ}$ Mn oxide coating

263.2: $30^{\circ}$ irregular, hairline

263.4-263.5: broken, angular

Ash-flow tuff, as preceding,

$0.7 \mathrm{ft}$ of E-core, blue painted.

$263.5-266.5$

3.0

(80.31-81.23)

Fractures and angles at following footages:

263.5: $35^{\circ} \mathrm{Mn}$ oxide, $45^{\circ} \mathrm{SiO}_{2}$ minerals

263.5-264.0: $10^{\circ}$ irregular

264.0-264.3: $10^{\circ}$ planar

264.3: Mn oxide coated

264.3-264.5: broken, angular, Debble size

264.5: $10^{\circ} \pm$ curved, $M n$ oxide coated

264.5-264.9: $20^{\circ}$ rough, hairline crack

265.1: $60^{\circ}$ and $45^{\circ}$ intersecting, Mn oxide

265.2: $15^{\circ} \mathrm{SiO}_{2}$ minerals and $0^{\circ}$ hackly

265.4-265.8: broken core, angular

No. Mn oxide coated

7

266.3: $35^{\circ} \mathrm{SiO}_{2}$ mineral coated, $60^{\circ}$

Mn oxide coated

266.5: Mn oxide coated

Ash-flow tuff, light yellowish brown, subtie gradational color change from overlying subunit

$266.5-272.0$

5.5

(hole penetrates successively older subunits

$(81.23-82.91)$

inward, assuming no major fault crossing,

inasmuch as general dip is east toward collar

of hole). Several larger flattened pumices,

as much as $5 \mathrm{~cm}$ long, a few with small litho-

physal cavities with vapor phase crystals. Pumice

flattening ratio, $3: 1$ to $5: 1$, moderately welded.

Pumice flattening within a few degrees of $0^{\circ}$.

Fractures at following footages:

266.5-266.8: $30^{\circ} \mathrm{Mn}$ oxide coated

266.8: $60^{\circ} \pm$ curved, $M n$ oxide coated

267.4: $30^{\circ} \pm \quad ", 25^{\circ} \pm$ irregular

267.6: $35^{\circ}, 40^{\circ} \mathrm{Mn}$ oxide coated

268.1: $45^{\circ}$ and $40^{\circ}$ intersecting, $M n$ oxide;

$20^{\circ}$ Mn oxide coating

268.3: $60^{\circ} \mathrm{Mn}$ oxide

268.5: $0^{\circ}, 65^{\circ} \mathrm{Mn}$ oxide, $45^{\circ}$ planar

268.6: $30^{\circ} \mathrm{SiO}_{2}$ minerals, $55^{\circ}$ and $45^{\circ}$ Mn oxide 
Description of Footage Interval (angles are measured from core axis)
Horizontal

Footage

(m)
Thickness of

Interval in

$\mathrm{ft}(\mathrm{m})$

268.8: $40^{\circ} \pm$ curved, $M n$ oxide

268.9: $45^{\circ}, 50^{\circ} \mathrm{Mn}$ oxide

268.9-269.3: irregular hairline cracks

269.5: hairline crack

269.6: $30^{\circ}$ rough surface

269.8: $60^{\circ}$ rough, $0^{\circ}$ planar

269.9: $50^{\circ}$ planar

270.1: $30^{\circ}$ planar

270.2: $40^{\circ} \mathrm{Mn}$ oxide coating

270.4: $45^{\circ}$ planar

270.7: $45^{\circ}$ hairline crack

270.8: $30^{\circ} \pm$ curved

271.0: $40^{\circ}$ irregular

271.1: $60^{\circ}$ planar

271.2: $45^{\circ}$ Mn oxide coated

271.5: $25^{\circ}$ rough surface, planar

271.5-272.0: broken, angular pieces

Ash-flow tuff, as preceding, mostly broken core,

$70 \%$ recovery. Fractures: $50^{\circ}, 30^{\circ}, 40^{\circ}, 35^{\circ}$,

$272.0-275.0 \quad 3.0$

$45^{\circ}, 15^{\circ}, 80^{\circ}$ planar, $0^{\circ}, 70^{\circ}, 45^{\circ}$ Mn oxide coated,

$(82.91-83.82) \quad(0.91)$

$90^{\circ} \mathrm{SiO}_{2}$ mineral coating

Ash-flow tuff, as preceding, but not broken. Pumice $2-5 \mathrm{~cm}$ with fiattening ratio

$2: 1$ to $4: 1$, and near $0^{\circ}$. Radio indicates

moderate welding or compaction.

Small $(0.5-\mathrm{cm})$ scattered white spherulites.

Fractures at

275.0: $70^{\circ}$ rough, $\mathrm{SiO}_{2}$ mineral coating

275.2: $70^{\circ}, 30^{\circ} \mathrm{Mn}$ oxide coating

275.2-275.4: $0^{\circ} \pm$ curved, Mn oxide coating

275.4: $40^{\circ}$ Mn oxide coating

275.4-275.8: $0^{\circ}$ planar

275.8: $50^{\circ}$ Dlanar

275.8-276.1: $5^{\circ}$ Mn oxide

276.1: $45^{\circ}, 35^{\circ}$ intersecting planar

276.1-276.8: solid piece, faint hairline cracks

276.8: $45^{\circ}$ rough, planar

277.1: $45^{\circ} \pm$ curved, Mn oxide

277.3-277.6: $25^{\circ}, 3$ parallel fractures

277.6: $40^{\circ}, 55^{\circ}$ Mn oxide coated

277.9: irregular hairline cracks

277.9-278.3: $10^{\circ}$ rough

278.1: $40^{\circ}$ rough

$278.5: 35^{\circ}$ "

$275.0-282.0$

7.0

$(83.82-85.95)$

279.0: $40^{\circ}$ 
Description of Footage Interval (angles are measured from core axis)

$\begin{array}{cl}\text { Horizontal } & \text { Thickness of } \\ \text { Footage } & \text { Interval in } \\ (\mathrm{m}) & \mathrm{ft}(\mathrm{m})\end{array}$

279.0-280.0: solid piece, faint hairline cracks 280.7: $45^{\circ}$ rough

280.9: $45^{\circ} \quad "$

281.0: $55^{\circ}, 60^{\circ}$ intersecting, rough

281.2: $60^{\circ}, 15^{\circ} \pm$ planar and curved

281.2-282.0: broken core, fracture surfaces:

$35^{\circ}, 50^{\circ}, 60^{\circ}, 55^{\circ}$ planar

$50^{\circ}, 40^{\circ}, 60^{\circ}, 55^{\circ}, 55^{\circ}$ Mn oxide

$5^{\circ}, 0^{\circ}$ hairline cracks

Ach-flow tuff, as preceding but somewhat more broken. Fractures:

282.0: $15^{\circ}, 25^{\circ} \mathrm{Mn}$ oxide coating

282.2: $80^{\circ} \mathrm{SiO}_{2}$ mineral coating

282.3: $35^{\circ} \mathrm{Mn}$ oxide; $30^{\circ}$ intersecting planar

282.5: $70^{\circ} \pm$ curved, $M n$ oxide

282.7: $40^{\circ}$ Mn oxide coated

282.8: $40^{\circ}, 35^{\circ}, 55^{\circ}$ Mn oxide

282.8-283.0: $35^{\circ}$ planar

283.0: $65^{\circ} \pm$ curved, $M n$ oxide coated

283.2: $40^{\circ}, 80^{\circ}$ closed cracks

283.4-283.7: $0^{\circ}+25^{\circ}$ curved, Mn oxide

283.6: $55^{\circ}$ Mn oxide coated

283.8: $90^{\circ} \pm$ curved, $M n$ oxide coated

283.8-284.0: $0^{\circ}+10^{\circ}$ curved, Mn oxide

284.0: $75^{\circ}$ rough surface

284.1: $45^{\circ}$ and $20^{\circ}$ calcite coated

284.1-284.4: $20^{\circ}, 0^{\circ} \mathrm{Mn}$ oxide coated

284.4: $30^{\circ} \mathrm{Mn}$ oxide coated

284.7: $0^{\circ}, 10^{\circ}, 80^{\circ}, 75^{\circ}$ calcite coated

284.8-285.2: $20^{\circ}$ calcite and $\mathrm{SiO}_{2}$ minerals

285.0: $90^{\circ} \pm$ Mn oxide coated

285.2: $45^{\circ}$ planar

285.4: $50^{\circ}$ calcite coated

285.4-285.9: 2 core pieces, hairline cracks

285.65-285.9: $15^{\circ} \mathrm{Mn}$ oxide coated

285.9: $60^{\circ}$ planar

286.0: $65^{\circ}$

Ash-flow tuff, as preceding. Fractures:

286.0-286.7: 1 piece, hairline cracks

286.7: $45^{\circ} \pm$ curved, Mn oxide coating

286.9: $50^{\circ}$ rough surface

286.9-287.0: broken core

287.0: $60^{\circ}$ and $65^{\circ}$ intersecting planar

287.3: $70^{\circ}$ planar

287.3-287.7: $15^{\circ}$ Mn oxide coating
$282.0-286.0 \quad 4.0$

$(85.95-87.17) \quad(1.22)$
$286.0-290.0 \quad 4.0$

$(87.17-88.39) \quad(1.22)$ 


\section{Description of Footage Interval \\ (angles are measured from core axis)}

$\begin{array}{cl}\text { Horizontal } & \text { Thickness of } \\ \text { Footage } & \text { Interval in } \\ (\mathrm{m}) & \mathrm{ft}(\mathrm{m})\end{array}$

287.8: $80^{\circ}$ Mn oxide coating

287.8-288.6: broken core; fractures:

$50^{\circ}, 85^{\circ}$ planar

$10^{\circ}, 55^{\circ}, 85^{\circ}, 0^{\circ}$ Mn oxide coating

$0^{\circ}, 65^{\circ}, 45^{\circ}$ salcite coated

288.6: $70^{\circ} \mathrm{SiO}_{2}$ mineral

288.6-288.9: $0^{\circ} \mathrm{Mn}$ oxide coated

288.9: $90^{\circ}$ Mn oxide coated

288.9-290.0: broken core, granules to cobble size, intricately fractured

Ash-flow tuff, as preceding. Fractures:

290.0-290.2: broken core, subangular

$290.0-293.0$

$(88.39-89.30)$

290.2: $60^{\circ} \mathrm{SiO}_{2}$ mineral, $30^{\circ}$ rough

290.4: $30^{\circ} \mathrm{Mn}$ oxide coated

290.7: $20^{\circ} \pm$ curved, Mn oxide; $40^{\circ}$ planar

290.8: $40^{\circ}$ Mn oxide coated

290.8-291.8: broken core, angular

$$
60^{\circ}, 45^{\circ}, 70^{\circ}, 45^{\circ} \text { planar }
$$

$5^{\circ}, 50^{\circ}, 60^{\circ}, 60^{\circ} \mathrm{Mn}$ oxide

291.8: $40^{\circ} \mathrm{Mn}$ oxide coating

291.8-292.0: $5^{\circ}$ rough surface

292.0: $65^{\circ}$ Mn oxide coated

292.3: $45^{\circ}$ planar

292.3-292.5: broken core, subangular

292.5: $45^{\circ} 1.5 \mathrm{~mm}$ calcite coated

292.6-292.9: $60^{\circ} 0.5-\mathrm{mm} \mathrm{SiO}_{2}$ mineral

Ash-flow tuff, color midway between pale yellowish

$293.0-295.0$

2.0

brown and light brown (5YR5/6) with moderate

brown (5YR4/4) flattened (3:1 to $4: 1)$ pumice

$(2-5 \mathrm{~cm})$ with spherulites in center, otherwise rock is similar to preceding. Core recovery 55\%.

Fractures at footages:

293.0-293.4: broken core, angular, Debble size

293.5 \pm : $40^{\circ} \mathrm{Mn}$ oxide coated

294 \pm : $80^{\circ}$ Mn oxide coated and $55^{\circ}$ calcite coated

294.5 $\pm: 45^{\circ}, 5^{\circ}, 30^{\circ} \mathrm{Mn}$ oxide coated

295士: $45^{\circ}, 30^{\circ}$ Mn oxide coated

Ash-flow tuff, as precedi.., fractures:

295.0-295.1: broken, angular

295.1: $60^{\circ}$ Mn oxide coated

$(89.30-89.92)$

295.3: $70^{\circ} 1 \mathrm{~mm}$ calcite coated

295.4: $65^{\circ}$ Mn oxide coated

295.4-296.1: broken core, angular, cobble size

295.0-299.0

4.0

$(89.92-91.14)$ 


\begin{tabular}{ccc} 
Description of Footage Interval & $\begin{array}{c}\text { Horizontal } \\
\text { Footage } \\
(\mathrm{m})\end{array}$ & $\begin{array}{l}\text { Thickness of } \\
\text { Interval in } \\
\mathrm{ft}(\mathrm{m})\end{array}$ \\
\hline
\end{tabular}

$70^{\circ}$ planar

$50^{\circ}, 40^{\circ}, 30^{\circ}, 5^{\circ}, 5^{\circ}, 45^{\circ}$ Mn oxide coated

296.3: $45^{\circ}$ Mn oxide coated

296.5: $55^{\circ} "$ " " "

296.6: $20^{\circ} "$ " $"$

296.8: $45^{\circ} "$ " " rough surface

296.9: $40^{\circ}$ planar, rough surface

$297.0: 50^{\circ}$

297.0-297.1: broken core, angular

297.1: $55^{\circ}$ Mn oxide coater

$297.3: 40^{\circ} "$ " " "

297.8: $20^{\circ} "$ " "

297.8-298.4: broken, angular, granule to pebble size

298.4: $40^{\circ}$ planar

298.7: $50^{\circ} \mathrm{SiO}_{2}$ mineral coated

298.9-299.0: broken core

Ash-flow tuff, light yellowish brown with

$299.0-302.0 \quad 3.0$

moderate brown 2-5-cm pumice flattened $3: 1$

$(91.14-92.05)$

to 4:1 (moderately welded) and small,

similarly flattened light-gray pumice, 3 to $8 \mathrm{~mm}$,

angle with core axis, $0-5^{\circ}$. Core broken in

cobble-size pieces. Fracture summary: $70^{\circ}, 35^{\circ}$

$70^{\circ}, 40^{\circ}, 55^{\circ}, 15^{\circ}, 40^{\circ}, 30^{\circ}$ planar; $80^{\circ}, 0^{\circ}, 15^{\circ}$,

$35^{\circ}, 80^{\circ}, 80^{\circ} \mathrm{Mn}$ oxide coated; $30^{\circ} \mathrm{SiO}_{2}$ mineral

coated.

Ash-flow tuff, as preceding, granule to cobble

size; fracture summary $5^{\circ}, 50^{\circ}, 45^{\circ}$ planar;

$40^{\circ}, 60^{\circ}, 75^{\circ}$ Mn oxide coated; $45^{\circ}, 80^{\circ}, 70^{\circ}$,

$302.0-305.0$

3.0

$40^{\circ}, 50^{\circ} \mathrm{SiO}_{2}$ mineral coated.

Ash-flow tuff, as preceding, fractures masked

$(92.05-92.96)$

by core breakage; $50 \%$ core recovery.

$305.0-307.0$

$(92.96-93.57)$

Ash-flow tuff, as preceding, pumice foliation,

$10^{\circ}$. Core intermittently fragmented and intact

$307.0-312.0$

with one piece (waxed) $0.6 \mathrm{ft}$. Fracture footages

(93.57-95.10) and angles below:

307.0-308.3: broken, angular, pebble-size

$85^{\circ}, 80^{\circ}, 85^{\circ}, 90^{\circ}$ planar surface

$85^{\circ}$ Mn oxide coated

308.3-308.7: $15^{\circ}$ Mn oxide coated

308.7: $55^{\circ}$ Dlanar surface

308.7-309.3: waxed core

309.3: $45^{\circ}$ planar surface 
Description of Footage Interval (angles are measured from core axis)
Horizontal

Footage

(m)
Thickness of Interval in ft $(m)$

309.4: $45^{\circ}$ planar surface

309.6: $40^{\circ}$ rough surface

309.6-310.0: irregular hairiine cracks

310.0: $35^{\circ}$ Mn oxide coated

310.0-310.1: broken core

310.1: $80^{\circ}$ planar

310.4: $30^{\circ}, 40^{\circ}, 60^{\circ}$ Mn oxide coated

310.6: $35^{\circ}$ Dlanar

310.7: $45^{\circ} \mathrm{SiO}_{2}$ mineral coated

311.0: $75^{\circ}$ planar

311.3: $45^{\circ}, 40^{\circ} \mathrm{Mn}$ oxide coated

311.3-312.0: broken, angular, pebble-cobble size

$85^{\circ}, 65^{\circ}$ Dlanar surface

$35^{\circ}, 10^{\circ}, 70^{\circ}, 15^{\circ} \mathrm{Mn}$ oxide coated

Ash-flow tuff, light yellowish brown, less broken,

$312.0-316.0$

4.0

with 4 bieces $>0.4 \mathrm{ft} ; 75 \%$ core recovery.

(95.10-96.32)

Fractures and angles at footages:

312.0-312.3: broken, angular

$35^{\circ} \mathrm{Mn}$ oxide coated

$35^{\circ}$ calcite coated

$60^{\circ}$ planar surface

312.3-312.8: 1 piece, pumice foliation $<5^{\circ}$

312.3: $50^{\circ} \times 20^{\circ}$ intersecting, calcite coated

312.4: irregular hairline crack

312.8: $40^{\circ}$ Dlanar

312.8-313.4: 1 piece with irregular hairline crack at 313.1 .

313.4: $60^{\circ}$ Dlanar, rough surface

313.4-313.6: broken core, angular $45^{\circ}$ planar

$30^{\circ}, 45^{\circ}$ Mn oxide coated

313.6-314.2: waxed core

314.2: $40^{\circ}$ Mn oxide coated

314.3: $55^{\circ}$ and $40^{\circ}$ intersecting $\mathrm{planar}$ fractures

314.7: $80^{\circ}$ rough hack ly, rare Mn oxide

314.8: $65^{\circ}$ Mn oxide coated

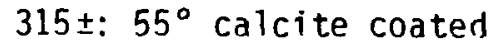

315.2: $50^{\circ} \mathrm{Mn}$ oxide coated

315.2-316.0: lost core

Ash-flow tuff, as preceding,

$40 \%$ recovery. Fractures: $75^{\circ}$ Dlanar; $35^{\circ}$

rough surface (probably others in lost core)

$316.0-316.5$

$(96.32-96.47)$

0.5

$(0.15)$ 
Description of Footage Interval (angles are measured from core axis)

$\begin{array}{cl}\text { Horizontal } & \text { Thickness of } \\ \begin{array}{c}\text { Footage } \\ (\mathrm{m})\end{array} & \text { Interval in } \\ & \mathrm{ft}(\mathrm{m})\end{array}$

$316.5-318.0 \quad 1.5$

$(96.47-96.93) \quad(0.46)$

Ash-flow tuff, as preceding. Fractures at

316.5: $70^{\circ}$ planar

316.6: $50^{\circ}$ "

316.9: $60^{\circ}, 35^{\circ}$ intersecting, Mn

oxide coating on both

317.1: $80^{\circ}$ Mn oxide coated

317.3: $65^{\circ}$ planar

317.6: $90^{\circ}, 75^{\circ}$ intersecting planar

318.0: $60^{\circ}$ planar

Ash-flow tuff, as preceding but more broken,

$318.0-323.0$

86\% recovery. Fracture footages and angles:

(96.93-98.45)

318.3: $55^{\circ}$ planar

318.4: $40^{\circ}$ Mn oxide coated

$318.6: 30^{\circ} "$ " " " "

313.7: $45^{\circ} "$ "

318.8: $90^{\circ}$ and $75^{\circ} \mathrm{Mn}$ oxide coated

318.9: $80^{\circ}$ Dlanar

319.0: $70^{\circ}-90^{\circ}$ curved irregularly, hackly

319.2-319.9: broken core, angular, pebble-size

$90^{\circ} 45^{\circ}, 35^{\circ}$ planar

$60^{\circ}, 75^{\circ}$ Mn oxide coated

319.9: $50^{\circ}$ planar

319.9-320.0: $0^{\circ}$ planar terminated at 320.0

320.0: $60-65^{\circ}$ irregular fracture

320.1: $50^{\circ}$ calcite coated

320.2-320.3: $70^{\circ}$ and $20-45^{\circ} 1.5-\mathrm{mm}$ calcite-fill

fracture makes sharp bend

320.3: $45^{\circ}$ planar truncates calcite fracture

320.5: $40^{\circ}$ and $45^{\circ}$ intersecting planars

320.6: $60^{\circ} \mathrm{Mn}$ oxide coated

320.7: $75^{\circ} "$ " " "

320.7-321.0: $20^{\circ}$ to $45^{\circ}$ curved hackly

321.0: $60-90^{\circ}$ curved rough surface

321.1: $75^{\circ}$ rough

321.1-321.2: broken core

321.2: $65^{\circ}$ Mn oxide coated

$30^{\circ}$ rough

321.7: $50^{\circ}$ Mn oxide coated

321.9: $70^{\circ} 1-\mathrm{mm}$ calcite coating

322.3: $70^{\circ}$ rough intersects $20^{\circ} \mathrm{Mn}$ oxide

322.3-323.0: assigned to lost core.

Ash-flow tuff, as preceding, three pebble-size

$323.0-333.0 \quad 10.0$

pieces, subrounded as if they had been in ball mill. (98.45-101.50) (3.05)

$4 \%$ core recovery. 
Description of Footage Interval (angles are measured from core axis)

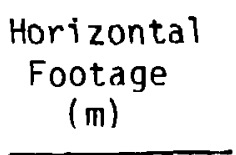

Thickness of Interval in $\mathrm{ft}(\mathrm{m})$

Ash-flow tuff, as preceding, except full recovery

$333.0-343.0$

10.0

and much less breakage. Large $(1-5-\mathrm{cm})$ and small

$(101.50-104.55)$

$(<1-\mathrm{cm})$ pumice lenticles, 0 to $10^{\circ}$. Fractures and angles at footages:

333.0-333.3: broken, subangular, pebble to cobble size.

333.3: $90^{\circ}$ Mn oxide coated

$45^{\circ} 1 / 2-m$ calcite filled

333.3.333.5: $10^{\circ}$ calcite coated

333.7: $70^{\circ} \times 70^{\circ}$ intersecting fractures; Mn oxide coated

334.0: $55^{\circ}$ Mn oxide coated

334.4: $75^{\circ}$ planar

334.6: $80^{\circ} \mathrm{SiO}_{2}$ mineral coated

334.6-335.1: broken, angular, pebble size

$40^{\circ}$ planar

$50^{\circ}, 85^{\circ}$ Mn oxide coated

$15^{\circ}, 40^{\circ}, 0^{\circ} \mathrm{SiO}_{2}$ mineral coated

335.1-335.8: 1 core piece, beveled

335.1: $70^{\circ} \mathrm{Mn}$ oxide cut by $40^{\circ} \mathrm{SiO}_{2}$ mineral

coated, in turn cut by $5^{\circ} \mathrm{SiO}_{2}$ mineral ccated

335.8: other end, $85^{\circ}$ Mn oxide coated

335.8-335.83: "poker crip" beveled by fractures

335.83: $90^{\circ}$ Mn oxide coated

335.83: 1 core piece, beveled

336.4: $90^{\circ}$ Mn oxide coated

336.6: $50^{\circ}$ rough planar

336.6-337.0: 1 core piece, ends beveled

337.0: $75^{\circ}$ Mn oxide coated

337.0-337.1: $45^{\circ}$ hairline crack broke open to

hackly fracture as core was pulled out of tray.

337.3: $45^{\circ}, 80^{\circ}$ hackly fracture

337.8: $70^{\circ} \mathrm{Mn}$ oxide coated

338.3: $70^{\circ}, 30^{\circ}$ hack ly

338.4: $55^{\circ}$ hackly

338.6: $40^{\circ}$ hackly, $\mathrm{Sin}_{2}$ mineral coated

$90^{\circ}$ hackly

338.6-338.8: $5^{\circ}$ Mn oxide coated

338.8: $65^{\circ}$ Mn oxide coated

339.3: core broken by hammer to fit in tray

339.3-339.9: waxed core

339.9: $90^{\circ} \pm$ curved

340.0: $80^{\circ}$ Mn oxide

340.2: irregular hairline crack

340.3: $45^{\circ}, 70^{\circ}$ rough

340.3-340.5: $10^{\circ} 0.5-m m$ calcite filled

340.5: $45^{\circ} \mathrm{Mn}$ oxide, $80^{\circ}$ rough 
APPENDIX B (cont)

Description of Footage Interval (angles are measured from core axis)

\begin{tabular}{cl} 
Horizontal & Thickness of \\
Footage & Interval in \\
$(\mathrm{m})$ & $\mathrm{ft}(\mathrm{m})$ \\
\hline
\end{tabular}

340.8: $80^{\circ}$ rough

341.2: $70^{\circ}$ planar

341.4: $75^{\circ} \mathrm{Mn}$ oxide coated

341.6: $75^{\circ}$ Mn oxide coated

341.6: $75^{\circ}$ planar

341.6-342.2: 1 piece, no fractures inside

342.2: $75^{\circ}$ Mn oxide, $45^{\circ}$ rough

342.2-342.4: $10^{\circ} \pm$ curved

$342.5: 45^{\circ}$ rough

342.5-342.7: $30^{\circ}$ planar

$342.7: 55^{\circ}$ rough

342.7-343.0: broken core, angular, pebble size

Ash-flow tuff, as preceding, but more broken;

$343.0-350.0$

7.0

core recovery $60 \%$.

$(104.55-106.68)$

Footages and angle of fractures:

343.0-343.2: broken

$90^{\circ}, 80^{\circ}$ planar

$80^{\circ}, 70^{\circ}, 40^{\circ} \mathrm{Mn}$ oxide coated

343.2: $45^{\circ}$ planar

$343.5: 85^{\circ}, 45^{\circ}, 45^{\circ}$ rough

343.7: $90^{\circ} \mathrm{Mn}$ oxide coated

344.0: $50^{\circ}$ " " "

344.0-344.8: broken, angular, pebble to cobble size:

$45^{\circ}, 60^{\circ}, 40^{\circ}, 5^{\circ}, 45^{\circ}, 30^{\circ}, 35^{\circ}$ rough

$35^{\circ}, 50^{\circ} \mathrm{Mn}$ oxide coated

344.8: $80^{\circ} \mathrm{SiO}_{2}$ mineral coated

$345.0: 60^{\circ} \pm$ curved

345.0-345.2: $0^{\circ}-20^{\circ}$ curved, rough

$345.2: 65^{\circ}$ rough

345.2-345.4: broken, angular, pebble size

345.4: $70^{\circ}$ rough

345.5: $45^{\circ}$ planar, intersects $60^{\circ} \pm$ curved

345.6: $35^{\circ} \mathrm{planar}$

345.9: $75^{\circ}$ rough

345.9-346.5: broken core, pebble to cobble size, angular to subangular, irregular fractures, possibly drilling induced

346.5-346.7: $30^{\circ}$ rough, bevels end of core piece

347.0: broken like 345.9-346.5

347.7-350: assigned to lost core

Ash-flow tuff, as preceding, but mostly broken core, $350.0-353.0 \quad 3.0$ granule to cobble size. Core recovery $87 \%$.

Fractures and angles:

$40^{\circ}, 20^{\circ}, 80^{\circ}, 55^{\circ}, 70^{\circ}$,

$75^{\circ}, 50^{\circ}, 0^{\circ}, 40^{\circ}, 40^{\circ}$ rough surface 
$50^{\circ}, 10^{\circ}, 20^{\circ}, 0^{\circ}, 30^{\circ}$ Mn oxide coated

$45^{\circ}, 80^{\circ}, 55^{\circ}$ planar

Ash-flow tuff, as preceding, $75 \%$ core recovery;

$353.0-355.0$

fractures:

$45^{\circ}, 30^{\circ}, 90^{\circ}, 70^{\circ}, 30^{\circ}, 40^{\circ}, 75^{\circ}$,

$55^{\circ}, 65^{\circ}, 70^{\circ}$ rough surface

$35^{\circ} \mathrm{SiO}_{2}$ mineral coated

Ash-flow tuff, as preceding, except for 2

$355.0-363.0$

beveled $0.35-\mathrm{ft}$ pieces. Core recovery,

91\%; fractures:

355-356.7: broken angular, debble size

$0^{\circ}, 55^{\circ}$ Dlanar

$45^{\circ}, 0^{\circ}, 90^{\circ}, 20^{\circ}, 45^{\circ}, 40^{\circ}, 75^{\circ}, 45^{\circ}$ rough

356.7: $10^{\circ}$ Mn oxide coated

355.7-357.05: 1 core piece beveled

357.05: $85^{\circ}$ rough

357.05-357.4: 1 core piece beveled

357.4: $40^{\circ}$ rough bevel of above

357.4-359.5: broken, angular, pebble to

cobble size

$40^{\circ}, 40^{\circ}, 40^{\circ}$ (2 parallel) $30^{\circ}, 40^{\circ}$ planar

$45^{\circ}, 40^{\circ}, 5^{\circ}, 75^{\circ}, 35^{\circ}, 0^{\circ}, 60^{\circ}$ rough

$20^{\circ}, 25^{\circ} \mathrm{SiO}_{2}$ mineral coated

$30^{\circ}, 0^{\circ}$ Mn oxide coated

$359.5: 80^{\circ} \pm$ curved, $50^{\circ}$ planar

359.6: $50^{\circ} \pm$ curved

359.6-359.8: broken core

359.8: $90^{\circ} \pm$ irregular, $20^{\circ}$ rough surface

360.0: $70^{\circ}$ curved

360.0-360.1: broken core

360.1: $55^{\circ} \mathrm{SiO}_{2}$ mineral coated

360.3: $80^{\circ} \pm$ curved

360.6: $50^{\circ} \mathrm{Mn}$ oxide coated

360.7: $90^{\circ} \pm$ curved

360.8: $45^{\circ}$ Dlanar

360.7-360.9: $5^{\circ} \mathrm{SiO}_{2}$ mineral coated

360.9: $50^{\circ}$ rough

360.9-362.3: broken core, angular, pebble size

$45^{\circ}, 65^{\circ}$ planar

$45^{\circ}, 70^{\circ}, 65^{\circ}, 45^{\circ}$ rough surface

$50^{\circ}, 5^{\circ}$ Mn oxide coating

$5^{\circ} \mathrm{SiO}_{2}$ mineral coated, same fracture as 360.7-360.9

(The two $5^{\circ}$ fractures run the length of this core

interval and may have caused the breakage)

362.3: $85^{\circ}$ rough 
Horizont.al

Footage

(m)
Thickness of Interval in ft $(\mathrm{m})$

362.5: $50^{\circ}$ rough

362.5-363.0: assumed core loss

Ash-flow tuff, grayish orange pink (5YR 5/2), $363.0-368.0$ slight change in color from preceding. Pumice lenticles, rare, indistinct. Core less broken than 353.0-363.0 run, $100 \%$ recovery. Fractures and angles at following footages:

363.0-363.3: broken core $35^{\circ}$ planar $30^{\circ}, 75^{\circ}$ rough surface $30^{\circ} \mathrm{SiO}_{2}$ mineral coated

363.3: $35^{\circ}$ planar

363.6: $50^{\circ}$ rough

363.8: $45^{\circ} "$

$364.0: 50^{\circ}, 20^{\circ}$ rough

364.2 : $35^{\circ}$ rough

364.3: $80^{\circ}$

364.3-365.1: 1 piece, no fractures

$365.1: 55^{\circ} \pm$ and $85^{\circ} \pm$ intersecting curving fractures

365.2: $45^{\circ}$ Mn oxide coated

365.25: $35^{\circ} 0.5-\mathrm{mm} \mathrm{Si0} \mathrm{z}_{2}$ mineral veinlet

365.5: $40^{\circ}$ planar

365.7: $45^{\circ}$ rough surface

365.9: $40^{\circ}$ planar

365.9-366.6: broken core, several fractures;

$5^{\circ} \mathrm{SiO}_{2}$ mineralized fracture only one measurable 366.6: $35^{\circ}$ planar, $40^{\circ} \pm$ curved

366.9: $60^{\circ}$ rough

367.3: $40^{\circ}, 40^{\circ}$ two parallel planar, $1 \mathrm{~cm}$ apart

367.5: $30^{\circ}$ planar

367.8: $70^{\circ}$ Mn oxide coated

367.8-368.0: broken core, $20^{\circ}$ rough

Ash-flow tuff, color changes back to light

yellowish brown, still hard, dense, as

$368.0-378.0$

10.0

preceding; pumice lenticles small $(<1 \mathrm{~cm})$,

indistinct, except for sparse larger $(1-5-\mathrm{cm})$

pumice with vapor phase crystals and spherulites.

Fractures and angles at following footages:

368.0-368.1: broken core

368.1: $65^{\circ}, 35^{\circ} \mathrm{Mn}$ oxide coated

368.3: $35^{\circ}$ rough

368.4: $10^{\circ} \mathrm{Mn}$ oxide coated

368.4-368.5: broken core

368.5: $60^{\circ}$ rough surface

$368.6: 75^{\circ}$ 
Description of Footage Interval (angles are measured from core axis)
Horizontal

Footage

(m)
Thickness of

Interval in

$\mathrm{ft}(\mathrm{m})$

368.8: $35^{\circ} \mathrm{Mn}$ oxide coated

$5^{\circ} \mathrm{SiO}_{2}$ mineral coated

368.8-369.0: $0^{\circ}$ Mn oxide coated

369.0-370.2: broken core, angular, pebble size

$75^{\circ}$ planar

$30^{\circ}, 90^{\circ}, 65^{\circ}, 85^{\circ}$ rough surface

$20^{\circ}, 90^{\circ}, 35^{\circ}, 45^{\circ}$ Mn oxide coated

$370.2: 70^{\circ}$ rough surface

370.5: $65^{\circ}$ " $370.55: 70^{\circ}$ Mn oxide

370.7: $70^{\circ} \mathrm{SiO}_{2}$ mineral coating

370.7-371.1: 1 piece with irregular hairline crack

371.1 : $60^{\circ}$ rough surface

$371.1-371.3: 30^{\circ}$ rough

371.3: $50^{\circ}$ planar

371.5: $65^{\circ}, 35^{\circ}$ rough

371.5-371.7: $5^{\circ} \mathrm{Mn}$ oxide coated

371.7-372.4: broken zone, subangular, pebble

size; $5^{\circ} \mathrm{Mn}$-oxide-coated fracture continues

through zone, intersected by a few low-

angle fractures.

372.4: $45^{\circ}$ rough surface

372.6: $90^{\circ} \pm$ curved surface

372.9: $90^{\circ} \pm$ " "

373.0: $70^{\circ}$ rough "

373.2: $90^{\circ} \pm$ curved "

373.3: $85^{\circ}$ rough "

373.4: $60^{\circ} "$ "

373.5: $90^{\circ} \pm "$ "

373.8: $90^{\circ}+$ curved "

373.8-374.1: broken core; fractures:

$0^{\circ}, 90^{\circ}, 40^{\circ}, 90^{\circ}$ rough surface

374.1: $70^{\circ}, 40^{\circ}$ rough

374.4: $90^{\circ} \pm$ curved surface

374.4-374.9: 1 core piece--no fractures

374.9: $50^{\circ} \times 50^{\circ}$, intersecting, rough

$375.1: 90^{\circ} \pm$ curved

$375.1-375.3: 10^{\circ}, 50^{\circ}, 70^{\circ}, 90^{\circ}$ rough

375.3: $60^{\circ}$ rough

375.3-375.7: 1 core diece--no fractures

375.7: $80^{\circ} \pm$ curved

$375.8: 45^{\circ}, 55^{\circ}$ rough surface
$376.1: 25^{\circ}, 70^{\circ}$ "

376.3: $30^{\circ} \mathrm{SiO}_{2}$ mineral coated

376.6: $35^{\circ}, 0^{\circ}-20^{\circ}$ irregular

376.7: $45^{\circ}$ calcite coated

$377.0: 55^{\circ} \mathrm{SiO}_{2}$ mineral coated, rough 
Descriotion of Footage Interval (angles are measured from core axis)
Horizontal

Footage

(m)
Thickness of Interval in $\mathrm{ft}(\mathrm{m})$

377.0-377.3: $0^{\circ}$ rough surface

$377.3: 75^{\circ}$ rough surface

377.5: $80^{\circ}$ planar, intersected by

$80^{\circ}$, opposite direction

377.5-377.7: $10^{\circ}$ rough surface

$377.7: 85^{\circ}$ rough

377.7-378.0: broken core, small pebble size.

Ash-flow tuff, color midway between grayish orange dink (5YR7/2) and pale yellowish brown (10YR6/2), similar to light yellowish brown core toward collar, dense, hard, several pieces of core 0.4 to $1.6 \mathrm{ft}$ in length. Small $(<1-\mathrm{cm})$ light gray pumice indistinct and sparse larger (1-5-cm) flattened (3:1 ratio) pumice subparallel to core axis. Detailed footages and angles of fractures follow:

378.0: $65^{\circ}$ Mn oxide coated

378.3: $80^{\circ}$ rough planar surface

$378.55: 45^{\circ}$

378.55-378.9: $5^{\circ} 1-\operatorname{mm}$ calcite coating

378.9: $50^{\circ} 1 / 2-1-\mathrm{mm}$ calcite coating intersects $5^{\circ} 1-\pi m$ calcite

379.3: $80^{\circ}$ rough

379.3-379.7: waxed core

379.7-380.05:

$380.05: 80^{\circ} \pm$ curved, rough

380.2: $45^{\circ} \mathrm{SiO}_{2}$ mineral coated

380.2-380.4: $30^{\circ} \pm$ curved, rough

380.7: rounded end of $0.5-\mathrm{ft}$ core from 380.2-380.7 $65^{\circ}$ planar at end of following core piece

380.9: $60^{\circ}$, truncated by $50^{\circ}$ rough

$381.1: 65^{\circ}$ rough surface

$381.3: 90^{\circ} "$ "

$381.5: 90^{\circ} \quad " 11$

$381.7: 55^{\circ} "$ "

381.7-383.3: 1 core piece

382.3-382.8: $70^{\circ} \mathrm{SiO}_{2}$ mineral filling crinkly fracture

$383.3: 80^{\circ}$ rough surface

$383.8: 75^{\circ}$ rough, cuts $40^{\circ} \pm$ curved

$384.05: 80^{\circ}$ rough

384.2: $50^{\circ} "$ "truncates $55^{\circ}$ rough

384.3: $70^{\circ} \mathrm{SiO}_{2}$ mineral coating

$384.5: 35^{\circ}$ rough, truncates $70^{\circ}$ rough
$378.0-388.0 \quad 10.0$

$(115.21-118.26) \quad(3.05)$ 
Description of Footage Interval (angles are measured from core axis)
Horizontal

Footage

(m)
Thickness of Interval in $\mathrm{ft}(\mathrm{m})$

384.5-385.3: 1 core piece with both ends beveled; no internal fractures. Pumice lenticles make $5-10^{\circ}$ with core axis--no fractures

385.3: $30^{\circ} \pm$ rough, truncates $20^{\circ}$ rough

385.6: $35^{\circ} \pm$ curved, rough

385.6-385.9: brcken core, mostly angular chips from intersection of fractures logged at 385.6 and 385.9

385.9: $35^{\circ}$ Mn oxide coated truncates $60^{\circ}$ rough planar fracture.

386.1: $75^{\circ}$ Mn oxide coated $20^{\circ}-65^{\circ}$ curved, Mn oxide coated

386.3: $70^{\circ}$ Mn oxide coated

386.3-386.8: unfractured core piece

386.8: $35^{\circ}$ rough, truncates $70^{\circ}$ rough

387.1: $70^{\circ}$ rough, wavy, may be drilling induced

387.1-387.4: waxed core

$387.4: 90^{\circ}$ rough

$387.4585^{\circ} \mathrm{Mn}$ oxide coated

387.9: $45^{\circ}$ rough, intersects

$388.0: 60^{\circ}$

Ash-flow tuff, color masked by rust from core $388.0-400.0(T D) 12.0$ barrel. Because of difficulty in removing core from barrel, part of core was not removed for 24 h. Core recovery $50 \%$ and consists of 17 pieces ranging from 0.2 to $0.4 \mathrm{ft}$. Core pieces are subangular to subrounded on ends, probably an artifact of being stuck in barrel. A few rough and planar surfaces are burnished with hardness greater than steel. Many fractures may have been in lost core. Following fractures and angles measured:

$$
\begin{aligned}
& 20^{\circ}, 45^{\circ}, 85^{\circ}, 75^{\circ}, 45^{\circ}, 65^{\circ}, 80^{\circ} \text { rough surface } \\
& 50^{\circ}, 30^{\circ} \text { Mn oxide coated } \\
& 75^{\circ}, 70^{\circ}, 65^{\circ} \text { planar } \\
& 50^{\circ} \mathrm{SiO}_{2} \text { mineral coated }
\end{aligned}
$$

Four samples submitted for density measurement are $2.21,2.23,2.21$, and $2.21 \mathrm{~g} / \mathrm{cm}^{3}$ (see text). 
[In the following calculations the "dip" is the dihedral angle between the plane of the fracture and a plane perpendicular to the core axis, analogous to the relation in a vertical hole in which this measurement represents the true dip. The dip parameter used in Appendix $C$ is for calculational purposes and is the complement of the "dips" for corresponding footages in Appendix $B$, in which only the high-angle dips $\left(>70^{\circ}\right)$ approximate the true dip and a northerly strike (because the hole was drilled westerly). 1

TABLE C-1. Calculation of Fracture Frequency per Cubic Meter UE $-25 \mathrm{h \# l}-209.0$ to $216.0 \mathrm{ft}$

\begin{tabular}{|c|c|c|c|c|c|c|}
\hline Did & $1 / \cos$ & $\#$ Fract & Product & Sum & Footage & \\
\hline 75.0 & 3.9 & 1.0 & 3.9 & 3.9 & $209.0-209.3$ & \\
\hline 15.0 & 1.0 & 1.0 & 1.0 & 4.9 & $209.0-209.3$ & \\
\hline 30.0 & 1.2 & 1.0 & 1.2 & 6.1 & $209.0-209.3$ & \\
\hline 30.0 & 1.2 & 1.0 & 1.2 & 7.2 & $209.4-209.7$ & \\
\hline 20.0 & 1.1 & 1.0 & 1.1 & 8.3 & $209.4-209.7$ & \\
\hline 55.0 & 1.7 & 1.0 & 1.7 & 10.0 & $209.4-209.7$ & \\
\hline 50.0 & 1.6 & 1.0 & 1.6 & 11.6 & $209.7-209.9$ & \\
\hline 25.0 & $\begin{array}{l}1.0 \\
1.1\end{array}$ & 1.0 & 1.1 & 12.7 & $209.7-209.9$ & \\
\hline 45.0 & 1.4 & 4.5 & 6.4 & 19.0 & $209.9-210.6$ & broken core \\
\hline 40.0 & 1.3 & 1.0 & 1.3 & 20.3 & 210.8 & \\
\hline 0.0 & 1.0 & 0.0 & 0.0 & 20.3 & $210.8-210.9$ & broken core \\
\hline 45.0 & 1.4 & 1.0 & 1.4 & 21.8 & $210.9-211.3$ & \\
\hline 85.0 & 11.5 & 1.0 & 11.5 & 33.2 & $210.9-211.3$ & \\
\hline 45.0 & 1.4 & 2.0 & 2.8 & 36.1 & $211.3-211.45$ & \\
\hline 30.0 & 1.2 & 2.0 & 2.3 & 38.4 & $211.45-211.6$ & \\
\hline 45.0 & 1.4 & 1.0 & 1.4 & 39.8 & $211.45-211.6$ & \\
\hline 65.0 & 2.4 & 1.0 & 2.4 & 42.2 & $211.45-211.6$ & \\
\hline 50.0 & 1.6 & 2.0 & 3.1 & 45.3 & $211.6-211.8$ & \\
\hline 35.0 & 1.2 & 1.0 & 1.2 & 46.5 & $211.6-211.8$ & \\
\hline 80.0 & 5.8 & 1.0 & 5.8 & 52.2 & $211.6-211.8$ & \\
\hline 45.0 & 1.4 & 2.0 & 2.8 & 55.1 & $211.8-2.12 .5$ & \\
\hline 50.0 & 1.5 & 1.0 & 1.6 & 56.6 & $211.8-212.5$ & \\
\hline 0.0 & 1.0 & 0.0 & 0.0 & 56.6 & $212.5-212.6$ & broken core \\
\hline 10.0 & 1.0 & 1.0 & 1.0 & 57.6 & 212.6 & \\
\hline 50.0 & 1.6 & 1.0 & 1.6 & 59.2 & 212.9 & \\
\hline 75.0 & 3.9 & 1.0 & 3.9 & 63.1 & 212.9 & \\
\hline 80.0 & 5.8 & 1.0 & 5.8 & 68.8 & $212.9-213.5$ & \\
\hline 45.0 & 1.4 & 2.0 & 2.8 & 71.6 & & irregular cracks \\
\hline 50.0 & 1.6 & 1.0 & 1.6 & 73.2 & $213.5-213.7$ & \\
\hline 40.0 & 1.3 & 1.0 & 1.3 & 74.5 & $213.5-213.7$ & \\
\hline 15.0 & 1.0 & 1.0 & 1.0 & 75.5 & $213.5-213.7$ & \\
\hline 45.0 & 1.4 & 7.0 & 9.9 & 85.4 & $213.7-216.0$ & broken core \\
\hline
\end{tabular}

Footage interval 7.0.

Fractures der cubic meter 50 .

Fractures der linear meter 21 . 


\section{APPENDIX C (cont)}

TABLE C-2. Calculation of Fracture Frequency per Cubic Meter UE $-25 \mathrm{~h} \# 1-221.0$ to $224.5 \mathrm{ft}$

\begin{tabular}{|c|c|c|c|c|c|c|}
\hline Dip & $1 / \cos$ & \# Fract. & Product & Sum & Footage & \\
\hline 40.0 & 1.3 & 1.0 & 1.3 & 1.3 & $221.0-224.5$ & broken core \\
\hline 55.0 & 1.7 & 1.0 & 1.7 & 3.0 & $221.0-224.5$ & broken core \\
\hline 70.0 & 2.9 & 1.0 & 2.9 & 6.0 & $221.0-224.5$ & broken core \\
\hline 85.0 & 11.5 & 1.0 & 11.5 & 17.4 & $221.0-224.5$ & broken core \\
\hline 25.0 & 1.1 & 1.0 & 1.1 & 18.5 & $221.0-224.5$ & broken core \\
\hline 45.0 & 1.4 & 18.0 & 25.5 & 44.0 & $221.0-224.5$ & broken core \\
\hline
\end{tabular}

Footage interval 3.5 .

Fractures per cubic meter 51 .

Fractures per linear meter 22 .

TABLE C-3. Calculation of Fracture Frequency per Cubic Meter UE-25h\#1 - 224.5 to $232.0 \mathrm{ft}$

\begin{tabular}{|c|c|c|c|c|c|c|}
\hline Dip & $1 / \cos$ & \# Fract & Product & Sum & Footage & \\
\hline 45.0 & 1.4 & 1.0 & 1.4 & 1.4 & 224.5 & \\
\hline 55.0 & 1.7 & 1.0 & 1.7 & 3.2 & 224.7 & \\
\hline 85.0 & 11.5 & 1.0 & 11.5 & 14.6 & 224.9 & \\
\hline 55.0 & 1.7 & 1.0 & 1.7 & 16.4 & $225.2-225.5$ & \\
\hline 50.0 & 1.6 & 1.0 & 1.6 & 17.9 & $225.2-225.5$ & \\
\hline 40.0 & 1.3 & 1.0 & 1.3 & 19.2 & $225.2-225.5$ & \\
\hline 85.0 & 11.5 & 1.0 & 11.5 & 30.7 & $225.2-225.5$ & \\
\hline 45.0 & 1.4 & 8.0 & 11.3 & 42.0 & $225.5-226.4$ & broken \\
\hline 35.0 & 1.2 & 2.0 & 2.4 & 44.5 & $226.4-226.6$ & \\
\hline 45.0 & 1.4 & 7.5 & 10.6 & 55.1 & $226.6-228.0$ & broken \\
\hline 30.0 & 1.2 & 1.5 & 1.7 & 56.8 & $226.6-228.0$ & broken \\
\hline 50.0 & 1.6 & 1.0 & 1.6 & 58.4 & $226.6-228.0$ & broken \\
\hline 45.0 & 1.4 & 14.5 & 20.5 & 78.9 & $228.0-232.0$ & broken \\
\hline 85.0 & 11.5 & 2.5 & 28.7 & 107.5 & $228.0-232.0$ & broken \\
\hline 45.0 & 1.4 & 3.0 & 4.2 & 111.8 & $228.0-232.0$ & broken \\
\hline 75.0 & 3.9 & 1.0 & 3.9 & 115.7 & $228.0-232.0$ & broken \\
\hline 30.0 & 1.2 & 2.0 & 2.3 & 118.0 & $228.0-232.0$ & broken \\
\hline 55.0 & 1.7 & 1.0 & 1.7 & 119.7 & $228.0-232.0$ & broken \\
\hline 70.0 & 2.9 & 1.0 & 2.9 & 122.6 & $228.0-232.0$ & broken \\
\hline 60.0 & 2.0 & 1.0 & 2.0 & 124.6 & $228.0-232.0$ & broken \\
\hline 50.0 & 1.6 & 1.0 & 1.6 & 126.2 & $228.0-232.0$ & broken \\
\hline
\end{tabular}

Footage interval 7.5.

Fractures per cubic meter 68 .

Fractures Der 1 inear meter 24 . 
TABLE C-4. Calculation of Fracture Frequency per Cubic Meter $U E-25 h \# 1-232.0$ to $237.0 \mathrm{ft}$

\begin{tabular}{|c|c|c|c|c|c|c|}
\hline Dip & $1 / \cos$ & $\#$ Fract & Product & Sum & Footage & \\
\hline 45.0 & 1.4 & 1.5 & 2.1 & 2.1 & $232.0-232.3$ & broken co \\
\hline 50.0 & 1.6 & 1.0 & 1.6 & 3.7 & 232.3 & \\
\hline 85.0 & 11.5 & 1.0 & 11.5 & 15.2 & $232.3-232.7$ & \\
\hline 20.0 & 1.1 & 1.0 & 1.1 & 16.2 & 232.7 & \\
\hline 0.0 & 1.0 & 0.0 & 0.0 & 16.2 & $232.7-233.0$ & lost core \\
\hline 40.0 & 1.3 & 1.0 & 1.3 & 17.5 & 233.0 & \\
\hline 60.0 & 2.0 & 1.0 & 2.0 & 19.5 & 233.4 & \\
\hline 35.0 & $1 . ?$ & 1.0 & 1.2 & 20.7 & 233.9 & \\
\hline 25.0 & 1.1 & 1.0 & 1.1 & 21.8 & 234.0 & \\
\hline 85.0 & 11.5 & 1.0 & 11.5 & 33.3 & 234.1 & \\
\hline 25.0 & 1.1 & 1.0 & 1.1 & 34.4 & 234.2 & \\
\hline 15.0 & 1.0 & 1.0 & 1.0 & 35.5 & 234.5 & \\
\hline 50.0 & 1.5 & 1.0 & 1.6 & 37.0 & 234.5 & \\
\hline 45.0 & 1.4 & 1.5 & 2.1 & 39.1 & $234.5-234.8$ & broken co \\
\hline 20.0 & 1.1 & 1.0 & 1.1 & 40.2 & 234.8 & \\
\hline 50.0 & 1.6 & 1.0 & 1.6 & 41.8 & 235.2 & \\
\hline 0.0 & 1.0 & 0.0 & 0.0 & 41.8 & $235.2-235.4$ & lost core \\
\hline 50.0 & 1.6 & 1.0 & 1.6 & 43.3 & 235.4 & \\
\hline 25.0 & 1.1 & 1.0 & 1.1 & 44.4 & 235.6 & \\
\hline 25.0 & 1.1 & 1.0 & 1.1 & 45.5 & 235.7 & \\
\hline 45.0 & 1.4 & 5.0 & 7.1 & 52.6 & $235.7-236.5$ & broken cor \\
\hline 0.0 & 1.0 & 0.0 & 0.0 & 52.6 & $236.5-237.0$ & lost core \\
\hline
\end{tabular}

Footage interval 5.0.

Fractures per cubic meter 43.

Fractures per 1 inear meter 16 . 


\section{APPENDIX C (cont)}

TABLE C-5. Calculation of Fracture Frequency per Cubic Meter $U E-25 h \# 1-240.0$ to $245.0 \mathrm{ft}$

\begin{tabular}{|c|c|c|c|c|c|c|}
\hline Did & $1 / \cos$ & \# Fract & Product & Sum & Footage & \\
\hline 50.0 & 1.6 & 2.0 & 3.1 & 3.1 & $240.0-240.1$ & \\
\hline 45.0 & 1.4 & 2.0 & 2.8 & 5.9 & 240.3 & \\
\hline 60.0 & 2.0 & 1.0 & 2.0 & 7.9 & 240.6 & \\
\hline 45.0 & 1.4 & 2.0 & 2.8 & 10.8 & $240.6-241.0$ & \\
\hline 85.0 & 11.5 & 1.0 & 11.5 & 22.2 & $241.0-241.3$ & \\
\hline 45.0 & 1.4 & 1.0 & 1.4 & 23.7 & $241.0-241.3$ & \\
\hline 55.0 & 1.7 & 1.0 & 1.7 & 25.4 & $241.0-241.3$ & \\
\hline 50.0 & 1.6 & 1.0 & 1.6 & 27.0 & $241.0-241.3$ & \\
\hline 85.0 & 11.5 & 1.0 & 11.5 & 38.4 & $241.3-242.0$ & \\
\hline 70.0 & 2.9 & 1.0 & 2.9 & 41.4 & $241.3-242.0$ & \\
\hline 45.0 & 1.4 & 1.0 & 1.4 & 42.8 & $241.3-242.0$ & \\
\hline 80.0 & 5.8 & 1.0 & 5.8 & 48.5 & $241.3-242.0$ & \\
\hline 20.0 & 1.1 & 1.0 & 1.1 & 49.6 & $242.0-242.4$ & \\
\hline 70.0 & 2.9 & 1.0 & 2.9 & 52.5 & $242.0-242.4$ & \\
\hline 45.0 & 1.4 & 1.0 & 1.4 & 53.9 & $242.0-242.4$ & \\
\hline 50.0 & 1.6 & 1.0 & 1.6 & 55.5 & $242.0-242.4$ & \\
\hline 45.0 & 1.4 & 4.0 & 5.7 & 61.1 & $242.4-243.2$ & \\
\hline 20.0 & 1.1 & 1.0 & 1.1 & 62.2 & $242.4-243.2$ & \\
\hline 50.0 & 1.6 & 1.0 & 1.6 & 63.8 & $242.4-243.2$ & \\
\hline 45.0 & 1.4 & 1.0 & 1.4 & 65.2 & $243.2-243.5$ & \\
\hline 20.0 & 1.1 & 1.0 & 1.1 & 66.2 & $243.2-243.5$ & \\
\hline 85.0 & 11.5 & 1.0 & 11.5 & 77.7 & $243.2-243.5$ & \\
\hline 80.0 & 5.8 & 1.0 & 5.8 & 83.5 & $243.5-243.9$ & \\
\hline 35.0 & 1.2 & 1.0 & 1.2 & 84.7 & $243.5-243.9$ & \\
\hline 45.0 & 1.4 & 1.0 & 1.4 & 86.1 & $243.5-243.9$ & \\
\hline 50.0 & 1.6 & 1.0 & 1.6 & 87.7 & $243.9-244.3$ & \\
\hline 55.0 & 1.7 & 1.0 & 1.7 & 89.4 & $244.3-244.7$ & broken cor \\
\hline 30.0 & 1.2 & 1.0 & 1.2 & 90.6 & $244.3-244.7$ & broken core \\
\hline 85.0 & 11.5 & 2.0 & 22.9 & 113.5 & $244.3-244.7$ & broken core \\
\hline 0.0 & 1.0 & 0.0 & 0.0 & 113.5 & $244.7-245.0$ & lost core \\
\hline
\end{tabular}

Footage interval 5.0.

Fractures per cubic meter 92.

Fractures per linear meter 24 . 


\section{APPENDIX C (cont)}

TABLE C-6. Calculation of Fracture Frequency per Cubic Meter UE $-25 h \# 1-245.0$ to $249.0 \mathrm{ft}$

\begin{tabular}{|c|c|c|c|c|c|c|}
\hline Dip & $1 / \cos$ & \# Fract & Product & Sum & Footage & \\
\hline 45.0 & 1.4 & 1.0 & 1.4 & 1.4 & 245.0 & \\
\hline 0.0 & 1.0 & 0.0 & 0.0 & 1.4 & $245.25-245.55$ & waxed co \\
\hline 45.0 & 1.4 & 1.0 & 1.4 & 2.8 & $245.55-245.8$ & \\
\hline 55.0 & 1.7 & 1.0 & 1.7 & 4.6 & $245.55-245.8$ & \\
\hline 60.0 & 2.0 & 1.0 & 2.0 & 6.6 & $245.55-245.8$ & \\
\hline 45.0 & 1.4 & 3.0 & 4.2 & 10.8 & $246.0-246.3$ & \\
\hline 20.0 & 1.1 & 1.0 & 1.1 & 11.9 & $246.3-246.5$ & \\
\hline 45.0 & 1.4 & 1.0 & 1.4 & 13.3 & $246.3-246.5$ & \\
\hline 85.0 & 11.5 & 1.0 & 11.5 & 24.8 & $246.3-246.5$ & \\
\hline 85.0 & 11.5 & 1.0 & 11.5 & 36.2 & $246.5-247.2$ & \\
\hline 20.0 & 1.1 & 1.0 & 1.1 & 37.3 & $246.5-247.2$ & \\
\hline 15.0 & 1.0 & 1.0 & 1.0 & 38.3 & $246.5-247.2$ & \\
\hline 60.0 & 2.0 & 1.0 & 2.0 & 40.3 & $247.2-249.0$ & \\
\hline 30.0 & 1.2 & 1.0 & 1.2 & 41.5 & $247.2-249.0$ & \\
\hline 45.0 & 1.4 & 2.0 & 2.8 & 44.3 & $247.2-249.0$ & \\
\hline 35.0 & 1.2 & 2.0 & 2.4 & 46.8 & $247.2-249.0$ & \\
\hline
\end{tabular}

Footage interval 4.0.

Fractures per cubic meter 48 .

Fractures per linear meter 16. 


\section{APPENDIX C (cont)}

TABLE C-7. Calculation of Fracture Frequency per Cubic Meter $U E-25 h \# 1-249.0$ to $253.0 \mathrm{ft}$

\begin{tabular}{|c|c|c|c|c|c|c|}
\hline Dip & $1 / \cos$ & \# Fract & Product & Sum & Footage & \\
\hline 0.0 & 1.0 & 0.0 & 0.0 & 0.0 & $249.0-249.2$ & broken core \\
\hline 30.0 & 1.2 & 1.0 & 1.2 & 1.2 & 249.2 & \\
\hline 50.0 & 1.6 & 1.0 & 1.6 & 2.7 & 250.1 & \\
\hline 0.0 & 1.0 & 2.0 & 2.0 & 4.7 & $250.1-250.7$ & \\
\hline 45.0 & 1.4 & 1.0 & 1.4 & 6.1 & $250.1-250.7$ & \\
\hline 20.0 & 1.1 & 1.0 & 1.1 & 7.2 & $250.1-250.7$ & \\
\hline 50.0 & 1.6 & 1.0 & 1.6 & 8.7 & 250.7 & \\
\hline 85.0 & 11.5 & 1.0 & 11.5 & 20.2 & 250.8 & \\
\hline 45.0 & 1.4 & 1.0 & 1.4 & 21.6 & 251.2 & \\
\hline 85.0 & 11.5 & 1.0 & 11.5 & 33.1 & $251.2-252.4$ & \\
\hline 75.0 & 3.9 & 1.0 & 3.9 & 37.0 & 251.4 & \\
\hline 50.0 & 1.6 & 1.0 & 1.6 & 38.5 & 251.6 & \\
\hline 60.0 & 2.0 & 1.0 & 2.0 & 40.5 & 251.6 & \\
\hline 15.0 & 1.0 & 1.0 & 1.0 & 41.6 & 252.1 & \\
\hline 40.0 & 1.3 & 1.0 & 1.3 & 42.9 & 252.4 & \\
\hline 55.0 & 1.7 & 1.0 & 1.7 & 44.6 & 252.6 & \\
\hline 45.0 & 1.4 & 1.0 & 1.4 & 46.0 & 252.7 & \\
\hline 45.0 & 1.4 & 2.5 & 3.5 & 49.6 & $252.7-253.0$ & broken co \\
\hline
\end{tabular}

Footage interval 4.0.

Fractures per cubic meter 50 .

Fractures der linear meter 16 . 
TABLE C-8. Calculation of Fracture Frequency Der Cubic Meter UE $-25 \mathrm{~h} \# 1$ - 253.0 to $259.0 \mathrm{ft}$

\begin{tabular}{|c|c|c|c|c|c|c|}
\hline Dip & $1 / \cos$ & \# Fract & Product & Sum & Footage & \\
\hline 45.0 & 1.4 & 2.0 & 2.8 & 2.8 & $253.0-253.5$ & \\
\hline 45.0 & 1.4 & 1.0 & 1.4 & 4.2 & 253.6 & \\
\hline 30.0 & 1.2 & 1.0 & 1.2 & 5.4 & 253.7 & \\
\hline 45.0 & 1.4 & 1.0 & 1.4 & 6.8 & 253.9 & \\
\hline 50.0 & 1.6 & 1.0 & 1.6 & 8.4 & 254.0 & \\
\hline 45.0 & 1.4 & 2.0 & 2.8 & 11.2 & $254.0-255.0$ & broken core \\
\hline 85.0 & 11.5 & 2.0 & 22.9 & 34.1 & $254.0-255.0$ & broken core \\
\hline 50.0 & 1.6 & 1.0 & 1.6 & 35.7 & $254.0-255.0$ & broken core \\
\hline 80.0 & 5.8 & 1.0 & 5.8 & 41.5 & $254.0-255.0$ & broken core \\
\hline 25.0 & 1.1 & 1.0 & 1.1 & 42.6 & $254.0-255.0$ & broken core \\
\hline 45.0 & 1.4 & 1.0 & 1.4 & 44.0 & 255.3 & \\
\hline 50.0 & 1.6 & 1.0 & 1.6 & 45.5 & 255.5 & \\
\hline 55.0 & 1.7 & 1.0 & 1.7 & 47.3 & 255.7 & \\
\hline 45.0 & 1.4 & 1.0 & 1.4 & 48.7 & 255.8 & \\
\hline 55.0 & 1.7 & 1.0 & 1.7 & 50.4 & 256.0 & \\
\hline 80.0 & 5.8 & 1.0 & 5.8 & 56.2 & 256.5 & \\
\hline 10.0 & 1.0 & 1.0 & 1.0 & 57.2 & 256.8 & \\
\hline 80.0 & 5.8 & 1.0 & 5.8 & 63.0 & $256.9-257.2$ & \\
\hline 75.0 & 3.9 & 1.0 & 3.9 & 66.8 & 257.3 & \\
\hline 45.0 & 1.4 & 1.0 & 1.4 & 68.2 & 257.5 & \\
\hline 15.0 & 1.0 & 1.0 & 1.0 & 69.3 & 257.7 & \\
\hline 75.0 & 3.9 & 1.0 & 3.9 & 73.1 & $257.7-257.9$ & \\
\hline 50.0 & 1.6 & 1.0 & 1.6 & 74.7 & $257.7-257.9$ & \\
\hline 20.0 & 1.1 & 1.0 & 1.1 & 75.8 & $257.7-257.9$ & \\
\hline 45.0 & 1.4 & 2.0 & 2.8 & 78.6 & $257.9-259.0$ & broken core \\
\hline 85.0 & 11.5 & 2.0 & 22.9 & 101.5 & $257.9-259.0$ & broken core \\
\hline 50.0 & 1.5 & 1.0 & 1.6 & 103.1 & $257.9-259.0$ & broken core \\
\hline 80.0 & 5.8 & 1.0 & 5.8 & 108.9 & $257.9-259.0$ & broken core \\
\hline 25.0 & 1.1 & 1.0 & 1.1 & 110.0 & $257.9-259.0$ & broken core \\
\hline
\end{tabular}

Footage interval 6.0.

Fractures Der cubic meter 75 .

Fractures der 1 inear meter 19. 


\section{APPENDIX C (cont)}

TABLE C-9. Calculation of Fracture Frequency per Cubic Meter UE $-25 \mathrm{~h} \# 1-259.0$ to $263.5 \mathrm{ft}$

\begin{tabular}{|c|c|c|c|c|c|c|}
\hline Dip & $1 / \cos$ & \# Fract & Product & Sum & Footage & \\
\hline 35.0 & 1.2 & 1.0 & 1.2 & 1.2 & $259.0-259.5$ & \\
\hline $\begin{array}{l}70.0 \\
30\end{array}$ & 2.9 & 1.0 & $\begin{array}{l}2.9 \\
1.2\end{array}$ & $\begin{array}{l}4.1 \\
5.3\end{array}$ & $259.5-259.8$ & \\
\hline $\begin{array}{l}30.0 \\
70.0\end{array}$ & $\begin{array}{l}1.2 \\
2.9\end{array}$ & $\begin{array}{l}1.0 \\
1.0\end{array}$ & $\begin{array}{l}1.2 \\
2.9\end{array}$ & $\begin{array}{l}5.3 \\
8.2\end{array}$ & $\begin{array}{l}259.8 \\
259.8-260.4\end{array}$ & \\
\hline 40.0 & 1.3 & 1.0 & 1.3 & 9.5 & 260.6 & \\
\hline 45.0 & 1.4 & 1.0 & 1.4 & 10.9 & 260.7 & \\
\hline 40.0 & 1.3 & 1.0 & 1.3 & 12.2 & $261 . \bar{I}$ & \\
\hline 45.0 & 1.4 & 2.0 & 2.8 & 15.1 & $261.1-261.7$ & \\
\hline 10.0 & 1.0 & 1.0 & 1.0 & 16.1 & $261.7-261.9$ & \\
\hline 85.0 & 11.5 & 1.0 & 11.5 & 27.6 & $261.7-261.9$ & \\
\hline 85.0 & 11.5 & 1.0 & 11.5 & 39.0 & $261.9-262.1$ & \\
\hline 20.0 & 1.1 & 1.0 & 1.1 & 40.1 & $261.9 \cdot-262.1$ & \\
\hline 80.0 & 5.8 & 1.0 & 5.8 & 45.9 & 262.2 & \\
\hline 25.0 & 1.1 & 1.0 & 1.1 & 47.0 & 262.3 & \\
\hline 5.0 & 1.0 & 1.0 & 1.0 & 48.0 & 262.4 & \\
\hline 30.0 & 1.2 & 1.0 & 1.2 & 49.1 & 262.4 & \\
\hline 45.0 & 1.4 & 1.0 & 1.4 & 50.5 & 262.4 & \\
\hline 35.0 & 1.2 & 1.0 & 1.2 & 51.8 & 262.5 & \\
\hline 45.0 & 1.4 & 2.0 & 2.8 & 54.6 & $262.5-262.7$ & broken core \\
\hline 20.0 & 1.1 & 1.0 & 1.1 & 55.7 & 262.7 & \\
\hline 50.0 & 1.6 & 1.0 & 1.6 & 57.2 & 262.9 & \\
\hline 60.0 & 2.0 & 1.0 & 2.0 & 59.2 & 263.2 & \\
\hline 45.0 & 1.4 & 2.0 & 2.8 & 62.0 & $263.4-263.5$ & \\
\hline
\end{tabular}

Footage interval 4.5 .

Fractures per cubic meter 56.

Fractures per linear meter 19 . 
TABLE C-10. Calculation of Fracture Frequency per Cubic Meter UE $-25 n \# 1-263.5$ to $266.5 \mathrm{ft}$

\begin{tabular}{|c|c|c|c|c|c|c|}
\hline Dip & $1 / \cos$ & \# Fract & Profuct & Sum & Footage & \\
\hline 55.0 & 1.7 & 1.0 & 1.7 & 1.7 & 263.5 & \\
\hline 45.0 & 1.4 & 1.0 & 1.4 & 3.2 & 263.5 & \\
\hline 80.0 & 5.8 & 1.0 & 5.8 & 8.9 & $263.5-264.0$ & \\
\hline 80.0 & 5.8 & 1.0 & 5.8 & 14.7 & $264.0-264.3$ & \\
\hline 40.0 & 1.3 & 1.0 & 1.3 & 16.0 & 264.3 & \\
\hline 45.0 & 1.4 & 2.0 & 2.8 & 18.8 & $264.3-204.5$ & broken core \\
\hline 80.0 & 5.8 & 1.0 & 5.8 & 24.6 & 264.5 & \\
\hline 70.0 & $? .9$ & 1.0 & 2.9 & 27.5 & $264.5-264.9$ & \\
\hline 30.0 & 1.2 & 1.0 & 1.2 & 28.6 & 265.1 & \\
\hline 45.0 & 1.4 & 1.0 & 1.4 & 30.1 & 265.1 & \\
\hline 15.0 & 1.0 & 1.0 & 1.0 & 31.1 & 265.2 & \\
\hline 85.0 & 11.5 & 1.0 & 11.5 & 42.6 & 265.2 & \\
\hline 45.0 & 1.4 & 3.5 & 4.9 & 47.5 & $265.4-265.8$ & broken core \\
\hline 55.0 & 1.7 & 1.0 & 1.7 & 49.3 & $266.3-265.8$ & \\
\hline 30.0 & 1.2 & 1.0 & 1.2 & 50.4 & $266.3-265.8$ & \\
\hline 15.0 & 1.0 & 1.0 & 1.0 & 51.5 & 266.5 & \\
\hline
\end{tabular}

Footage interval 3.0.

Fractures der cubic meter 70 .

Fractures per linear meter 21 . 


\section{APPENDIX C (cont)}

TABLE C-11. Calculation of Fracture Frequency per Cubic Meter UE $-25 h \# 1-266.5$ to $272.0 \mathrm{ft}$

\begin{tabular}{|c|c|c|c|c|c|}
\hline Did & $1 / \cos$ & \# Fract & Product & Sum & Footage \\
\hline 60.0 & 2.0 & 1.0 & 2.0 & 2.0 & $266.5-266.8$ \\
\hline 30.0 & 1.2 & 1.0 & 1.2 & 3.2 & 266.8 \\
\hline 50.0 & 1.6 & 1.0 & 1.6 & 4.7 & 267.4 \\
\hline 65.0 & 2.4 & 1.0 & 2.4 & 7.1 & 267.4 \\
\hline 55.0 & 1.7 & 1.0 & 1.7 & 8.8 & 267.6 \\
\hline 50.0 & 1.6 & 1.0 & 1.6 & 10.4 & 267.6 \\
\hline 50.0 & 1.6 & 1.0 & 1.6 & 11.9 & 267.8 \\
\hline 45.0 & 1.4 & 1.0 & 1.4 & 13.3 & 268.1 \\
\hline 50.0 & 1.6 & 1.0 & 1.6 & 14.9 & 268.1 \\
\hline 70.0 & 2.9 & 1.0 & 2.9 & 17.8 & 268.1 \\
\hline 30.0 & 1.2 & 1.0 & 1.2 & 19.0 & 268.3 \\
\hline 85.0 & 11.5 & 1.0 & 11.5 & 30.5 & 268.5 \\
\hline 25.0 & 1.1 & 1.0 & 1.1 & 31.6 & 268.5 \\
\hline 45.0 & 1.4 & 1.0 & 1.4 & 33.0 & 268.5 \\
\hline 60.0 & 2.0 & 1.0 & 2.0 & 35.0 & 268.6 \\
\hline 35.0 & 1.2 & 1.0 & 1.2 & 36.2 & 268.6 \\
\hline 45.0 & 1.4 & 1.0 & 1.4 & 37.6 & 268.6 \\
\hline 50.0 & 1.6 & 1.0 & 1.6 & 39.2 & 268.8 \\
\hline 45.0 & 1.4 & 1.0 & 1.4 & 40.6 & 268.9 \\
\hline 40.0 & 1.3 & 1.0 & 1.3 & 41.9 & 268.9 \\
\hline 45.0 & 1.4 & 2.0 & 2.8 & 44.7 & $268.9-269.3$ \\
\hline 30.0 & 1.2 & 1.0 & 1.2 & 45.9 & 269.3 \\
\hline 45.0 & 1.4 & 1.0 & 1.4 & 47.3 & 269,5 \\
\hline 60.0 & 2.0 & 1.0 & 2.0 & 49.3 & 269.6 \\
\hline 30.0 & 1.2 & 1.0 & 1.2 & 50.4 & 269.8 \\
\hline 85.0 & 11.5 & 1.0 & 11.5 & 61.9 & 269.8 \\
\hline 40.0 & 1.3 & 1.0 & 1.3 & 63.2 & 269.9 \\
\hline 60.0 & 2.0 & 1.0 & 2.0 & 65.2 & 270.1 \\
\hline 50.0 & 1.6 & 1.0 & 1.6 & 66.8 & 270.2 \\
\hline 45.0 & 1.4 & 1.0 & 1.4 & 68.2 & 270.4 \\
\hline 45.0 & 1.4 & 1.0 & 1.4 & 69.6 & 270.7 \\
\hline 60.0 & 2.0 & 1.0 & 2.0 & 71.6 & 270.8 \\
\hline 50.0 & 1.6 & 1.0 & 1.6 & 73.2 & 271.0 \\
\hline 30.0 & 1.2 & 1.0 & 1.2 & 74.3 & 271.1 \\
\hline 45.0 & 1.4 & 1.0 & 1.4 & 75.7 & 271.2 \\
\hline 65.0 & 2.4 & 1.0 & 2.4 & 78.1 & 271.5 \\
\hline 45.0 & 1.4 & 2.0 & 2.8 & 80.9 & $271.5-272.0$ \\
\hline
\end{tabular}

Footage interval 5.5 .

Fractures per cubic meter 60 .

Fractures per linear meter 23. 


$$
\text { APPENDIX C ( cont) }
$$

TABLE C-12. Calculation of Fracture Frequency per Cubic Meter UE $-25 \mathrm{~h} \# 1-272.0$ to $275.0 \mathrm{ft}$

\begin{tabular}{|c|c|c|c|c|c|c|}
\hline Dip & $1 / \cos$ & \# Fract & Product & Sum & Footage & \\
\hline 40.0 & 1.3 & 1.0 & 1.3 & 1.3 & $272.0-275.0$ & broken core \\
\hline 45.0 & 1.4 & 3.0 & 4.2 & 5.5 & & \\
\hline 60.0 & 2.0 & 1.0 & 2.0 & 7.5 & & \\
\hline 50.0 & 1.6 & 1.0 & 1.6 & 9.1 & & \\
\hline 55.0 & 1.7 & 1.0 & 1.7 & 10.8 & & \\
\hline 75.0 & 3.9 & 1.0 & 3.9 & 14.7 & & \\
\hline 10.0 & 1.0 & 1.0 & 1.0 & 15.7 & & \\
\hline 85.0 & 11.5 & 1.0 & 11.5 & 27.2 & & \\
\hline 20.0 & 1.1 & 1.0 & 1.1 & 28.3 & & \\
\hline 0.0 & 1.0 & 1.0 & 1.0 & 29.3 & & \\
\hline
\end{tabular}

Footage interval 3.0.

Fractures per cubic meter 40 .

Fractures per linear meter 13. 
TABLE C-13. Calculation of Fracture Frequency per Cubic Meter UE $-25 \mathrm{h \# l}-275.0$ to $282.0 \mathrm{ft}$

\begin{tabular}{|c|c|c|c|c|c|c|}
\hline Dip & $1 / \cos$ & \# Fract & Product & Sum & Footage & \\
\hline 20.0 & 1.1 & 1.0 & 1.1 & 1.1 & 275.0 & \\
\hline 20.0 & 1.1 & 1.0 & 1.1 & 2.1 & 275.2 & \\
\hline 60.0 & 2.0 & 1.0 & 2.0 & 4.1 & 275.2 & \\
\hline 85.0 & 11.5 & 1.0 & 11.5 & 15.6 & $275.2-275.4$ & \\
\hline 50.0 & 1.6 & 1.0 & 1.6 & 17.2 & 275.4 & \\
\hline 85.0 & 11.5 & 1.0 & 11.5 & 28.6 & $275.4-275.8$ & \\
\hline 40.0 & 1.3 & 1.0 & 1.3 & 29.9 & 275.8 & \\
\hline 85.0 & 11.5 & 1.0 & 11.5 & 41.4 & $275.8-276.1$ & \\
\hline 45.0 & 1.4 & 1.0 & 1.4 & 42.8 & 276.1 & \\
\hline 55.0 & 1.7 & 1.0 & 1.7 & 44.6 & 276.1 & \\
\hline 45.0 & 1.4 & 1.0 & 1.4 & 46.0 & $276.1-276.8$ & \\
\hline 45.0 & 1.4 & 1.0 & 1.4 & 47.4 & 276.8 & \\
\hline 45.0 & 1.4 & 1.0 & 1.4 & 48.8 & 277.1 & \\
\hline 65.0 & 2.4 & 3.0 & 7.1 & 55.9 & $277.3-277.6$ & \\
\hline 50.0 & 1.6 & 1.0 & 1.6 & 57.5 & 277.6 & \\
\hline 35.0 & 1.2 & 1.0 & 1.2 & 58.7 & 277.6 & \\
\hline 45.0 & 1.4 & 1.0 & 1.4 & 60.1 & 277.9 & \\
\hline 80.0 & 5.8 & 1.0 & 5.8 & 65.9 & $277.9-278.3$ & \\
\hline 50.0 & 1.6 & 1.0 & 1.6 & 67.4 & 278.3 & \\
\hline 50.0 & 1.6 & 1.0 & 1.6 & 69.0 & 278.5 & \\
\hline 50.0 & 1.6 & 1.0 & 1.6 & 70.5 & 279.0 & \\
\hline 45.0 & 1.4 & 2.0 & 2.8 & 73.4 & $279.0-280.0$ & \\
\hline 20.0 & 1.1 & 1.0 & 1.1 & 74.4 & 280.0 & \\
\hline 45.0 & 1.4 & 1.0 & 1.4 & 75.8 & $280.0-280.7$ & \\
\hline 45.0 & 1.4 & 1.0 & 1.4 & 77.2 & 280.7 & \\
\hline 45.0 & 1.4 & 1.0 & 1.4 & 78.7 & 280.9 & \\
\hline 35.0 & 1.2 & 1.0 & 1.2 & 79.9 & 281.0 & \\
\hline 30.0 & 1.2 & 1.0 & 1.2 & 81.0 & 281.0 & \\
\hline 30.0 & 1.2 & 1.0 & 1.2 & 82.2 & 281.2 & \\
\hline 75.0 & 3.9 & 1.0 & 3.9 & 86.1 & 281.2 & \\
\hline 55.0 & 1.7 & 1.0 & 1.7 & 87.8 & $281.2-282.0$ & broken core \\
\hline 40.0 & 1.3 & 2.0 & 2.6 & 90.4 & & \\
\hline 30.0 & 1.2 & 2.0 & 2.3 & 92.7 & & \\
\hline 35.0 & 1.2 & 3.0 & 3.7 & 96.4 & & \\
\hline 50.0 & 1.6 & 1.0 & 1.6 & 97.9 & & \\
\hline 85.0 & 11.5 & 2.0 & 22.9 & 120.9 & & \\
\hline
\end{tabular}

Footage interval 7.0.

Fractures per cubic meter 70 . Fractures per 1 inear meter 21 . 
TABLE C-14. Calculation of Fracture Frequency per Cubic Meter UE $-25 h \# 1-282.0$ to $286.0 \mathrm{ft}$

\begin{tabular}{|c|c|c|c|c|c|}
\hline Dip & $1 / \cos$ & \# Fract & Product & Sum & Footage \\
\hline 75.0 & 3.9 & 1.0 & 3.9 & 3.9 & 282.0 \\
\hline 65.0 & 2.4 & 1.0 & 2.4 & 6.2 & 282.0 \\
\hline 10.0 & 1.0 & 1.0 & 1.0 & 7.2 & 282.2 \\
\hline 55.0 & 1.7 & 1.0 & 1.7 & 9.0 & 282.3 \\
\hline 60.0 & 2.0 & 1.0 & 2.0 & 11.0 & 282.3 \\
\hline 20.0 & 1.1 & 1.0 & 1.1 & 12.1 & 282.5 \\
\hline 50.0 & 1.6 & 1.0 & 1.6 & 13.6 & 282.7 \\
\hline 50.0 & 1.6 & 1.0 & 1.6 & 15.2 & 282.8 \\
\hline 55.0 & 1.7 & 1.0 & 1.7 & 16.9 & 282.8 \\
\hline 35.0 & 1.2 & 1.0 & 1.2 & 18.1 & 282.8 \\
\hline 65.0 & 2.4 & 1.0 & 2.4 & 20.5 & $282.8-283.0$ \\
\hline 25.0 & 1.1 & 1.0 & 1.1 & 21.6 & 283.0 \\
\hline 50.0 & 1.6 & 1.0 & 1.6 & 23.2 & 283.2 \\
\hline 10.0 & 1.0 & 1.0 & 1.0 & 24.2 & 283.2 \\
\hline 80.0 & 5.8 & 1.0 & 5.8 & 29.9 & $283.4-283.7$ \\
\hline 25.0 & 1.1 & 1.0 & 1.1 & 31.0 & 283.6 \\
\hline 0.0 & 1.0 & 1.0 & 1.0 & 32.0 & 283.8 \\
\hline 85.0 & 11.5 & 1.0 & 11.5 & 43.5 & $283.8-284.0$ \\
\hline 15.0 & 1.0 & 1.0 & 1.0 & 44.5 & 284.0 \\
\hline 45.0 & 1.4 & 1.0 & 1.4 & 46.0 & 284.1 \\
\hline 70.0 & 2.9 & 1.0 & 2.9 & 48.9 & 284.1 \\
\hline 70.0 & 2.9 & 1.0 & 2.9 & 51.8 & $284.1-284.4$ \\
\hline 85.0 & 11.5 & 1.0 & 11.5 & 63.3 & $284.1-284.4$ \\
\hline 60.0 & 2.0 & 1.0 & 2.0 & 65.3 & 284.4 \\
\hline 85.0 & 11.5 & 2.0 & 22.9 & 88.2 & 284.7 \\
\hline 10.0 & 1.0 & 1.0 & 1.0 & 89.2 & 284.7 \\
\hline 15.0 & 1.0 & 1.0 & 1.0 & 90.3 & 284.7 \\
\hline 70.0 & 2.9 & 1.0 & 2.9 & 93.2 & $284.8-285.2$ \\
\hline 0.0 & 1.0 & 1.0 & 1.0 & 94.2 & $284.8-285.2$ \\
\hline 45.0 & 1.4 & 1.0 & 1.4 & 95.6 & 285.2 \\
\hline 40.0 & 1.3 & 1.0 & 1.3 & 96.9 & 285.4 \\
\hline 45.0 & 1.4 & 2.0 & 2.8 & 99.7 & $285.4-285.9$ \\
\hline 75.0 & 3.9 & 1.0 & 3.9 & 103.6 & $285.4-285.9$ \\
\hline 30.0 & 1.2 & 1.0 & 1.2 & 104.8 & 285.9 \\
\hline 25.0 & 1.1 & 1.0 & 1.1 & 105.9 & 286.0 \\
\hline
\end{tabular}

Footage interval 4.0 .

Fractures per cubic meter 108 .

Fractures per linear meter 30 . 


\section{APPENDIX C (cont)}

TABLE C-15. Calculation of Fracture Frequency per Cubic Meter UE $-25 \mathrm{h \# l}-286.0$ to $293.0 \mathrm{ft}$

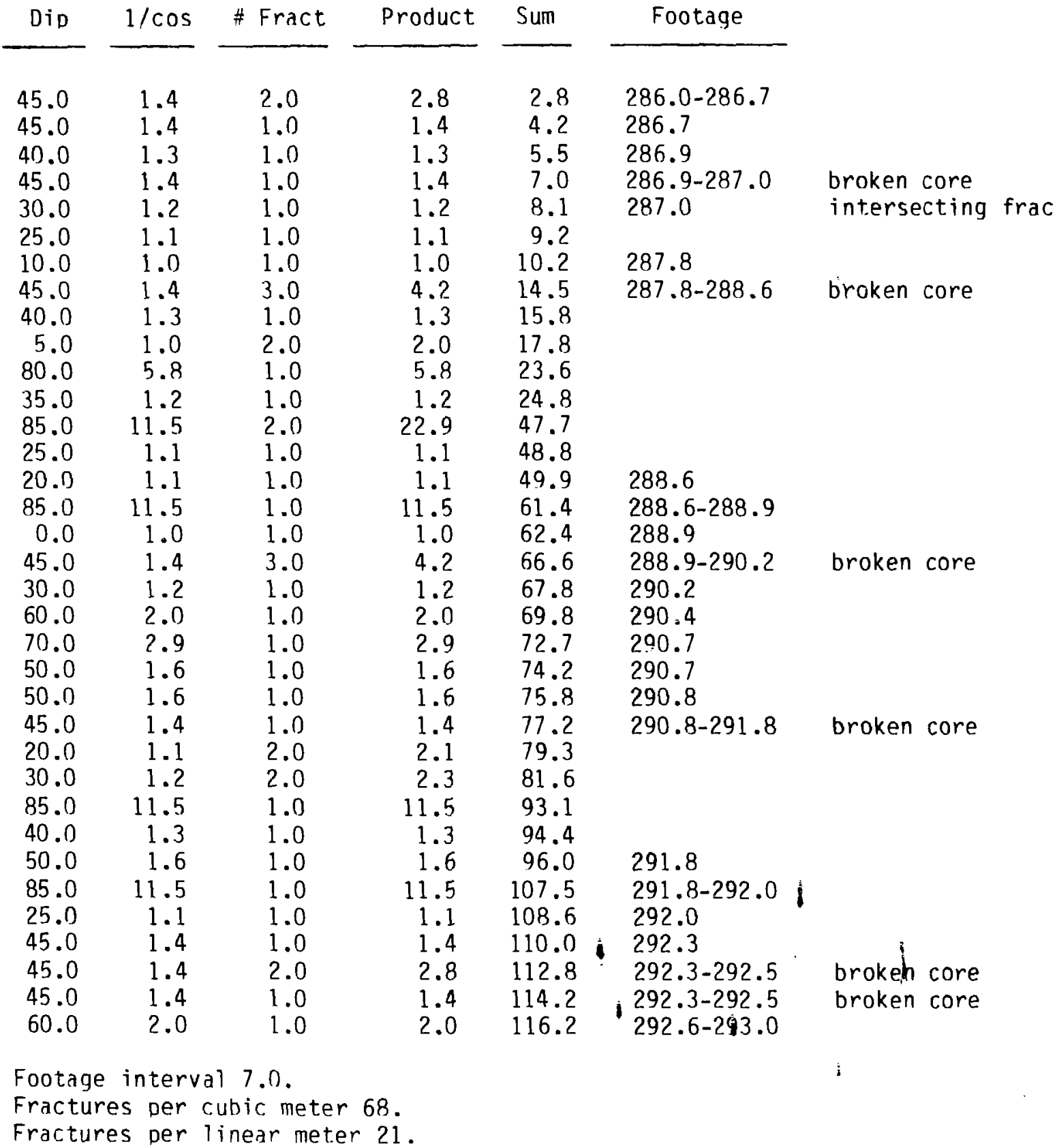


APPENDIX C (cont)

TABLE C-16. Calculation of Fracture Frequency Der Cubic Meter UE -25h\#1 - 293.0 to $299.0 \mathrm{ft}$

\begin{tabular}{|c|c|c|c|c|c|c|}
\hline Dip & $1 / \cos$ & \# Fract & Product & Sum & Footage & \\
\hline 45.0 & 1.4 & 4.0 & 5.7 & 5.7 & $293.0-293.4$ & \\
\hline 50.0 & 1.6 & 1.0 & 1.6 & 7.2 & 293.5 & \\
\hline 10.0 & 1.0 & 1.0 & 1.0 & 8.2 & 294.0 & \\
\hline 35.0 & 1.2 & 1.0 & 1.2 & 9.4 & 294.0 & \\
\hline 45.0 & 1.4 & 2.0 & 2.8 & 12.3 & $294.5-295.0$ & \\
\hline 60.0 & 2.0 & 2.0 & 4.0 & 16.3 & $294.5-295.0$ & \\
\hline 85.0 & 11.5 & 1.0 & 11.5 & 27.8 & & \\
\hline 45.0 & 1.4 & 1.0 & 1.4 & 29.2 & $295.0-295.1$ & broken core \\
\hline 30.0 & 1.2 & 1.0 & 1.2 & 30.3 & 295.1 & \\
\hline 20.0 & 1.1 & 1.0 & 1.1 & 31.4 & 295.3 & \\
\hline 25.0 & 1.1 & 1.0 & 1.1 & 32.5 & 295.4 & \\
\hline 85.0 & 11.5 & 1.0 & 11.5 & 44.0 & $295.4-296.1$ & broken core \\
\hline 20.0 & 1.1 & 1.0 & 1.1 & 45.0 & & \\
\hline 50.0 & 1.6 & 1.0 & 1.6 & 46.6 & & \\
\hline 60.0 & 2.0 & 1.0 & 2.0 & 48.6 & & \\
\hline 45.0 & 1.4 & 1.0 & 1.4 & 50.0 & & \\
\hline 40.0 & 1.3 & 1.0 & 1.3 & 51.3 & & \\
\hline 45.0 & 1.4 & 2.0 & 2.8 & 54.1 & 296.3 & \\
\hline 35.0 & 1.2 & 1.0 & 1.2 & 55.3 & 296.5 & \\
\hline 70.0 & 2.9 & 1.0 & 2.9 & 58.3 & 296.6 & \\
\hline 45.0 & 1.4 & 1.0 & 1.4 & 59.7 & 296.8 & \\
\hline 50.0 & 1.6 & 1.0 & 1.6 & 61.2 & 296.9 & \\
\hline 40.0 & 1.3 & 1.0 & 1.3 & 62.5 & 297.0 & \\
\hline 45.0 & 1.4 & 1.0 & 1.4 & 64.0 & $297.0-297.1$ & broken core \\
\hline 35.0 & 1.2 & 1.0 & 1.2 & 65.2 & 297.1 & \\
\hline 50.0 & 1.6 & 1.0 & 1.6 & 66.7 & 297.3 & \\
\hline 70.0 & 2.9 & 1.0 & 2.9 & 69.7 & 297.8 & \\
\hline 45.0 & 1.4 & 6.0 & 8.5 & 78.1 & $297.8-298.4$ & broken core \\
\hline 50.0 & 1.6 & 1.0 & 1.6 & 79.7 & 298.4 & \\
\hline 40.0 & 1.3 & 1.0 & 1.3 & 81.0 & 298.7 & \\
\hline 15.0 & 1.0 & 1.0 & 1.0 & 82.0 & 298.9 & \\
\hline 45.0 & 1.4 & 1.0 & 1.4 & 83.5 & $298.9-299.0$ & broken core \\
\hline
\end{tabular}

-Footage interval 6.0.

Fractures Der cubic meter 57.

Fractures per 1 inear meter 24 . 


\section{APPENDIX C (cont)}

TABLE C-17. Calculation of Fracture Frequericy per Cubic Meter UE $-25 \mathrm{~h} \# 1-299.0$ to $305.0 \mathrm{ft}$

\begin{tabular}{|c|c|c|c|c|c|c|}
\hline Did & $1 / \cos$ & \# Fract & Product & Sum. & Footage & \\
\hline 20.0 & 1.1 & 1.0 & 1.1 & 1.1 & $299.0-302.0$ & broken core \\
\hline 55.0 & 1.7 & 1.0 & 1.7 & 2.8 & & \\
\hline 50.0 & 1.6 & 1.0 & 1.6 & 4.4 & & \\
\hline 35.0 & 1.2 & 1.0 & 1.2 & 5.6 & & \\
\hline 75.0 & 3.9 & 1.0 & 3.9 & 9.4 & & \\
\hline 60.0 & 2.0 & 1.0 & 2.0 & 11.4 & & \\
\hline 10.0 & 1.0 & 1.5 & 1.5 & 13.0 & & \\
\hline 85.0 & 11.5 & 1.0 & 11.5 & 24.4 & & \\
\hline 75.0 & 3.9 & 1.0 & 3.9 & 28.3 & & \\
\hline 55.0 & 1.7 & 1.0 & 1.7 & 30.1 & & \\
\hline 60.0 & 2.0 & 1.0 & 2.0 & 32.1 & & broken core \\
\hline 45.0 & 1.4 & 10.0 & 14.1 & 46.2 & & hairline cracks \\
\hline 85.0 & 11.5 & 1.0 & 11.5 & 57.7 & $302.0-305.0$ & broken core \\
\hline 40.0 & 1.3 & 1.0 & 1.3 & 59.0 & & \\
\hline 45.0 & 1.4 & 1.0 & 1.4 & 60.4 & & \\
\hline 50.0 & 1.6 & 1.0 & 1.6 & 61.9 & & \\
\hline 30.0 & 1.2 & 1.0 & 1.2 & 63.1 & & \\
\hline 15.0 & 1.0 & 1.0 & 1.0 & 64.1 & & \\
\hline 45.0 & 1.4 & 1.0 & 1.4 & 65.5 & & \\
\hline 10.0 & 1.0 & 1.0 & 1.0 & 66.6 & & \\
\hline 20.0 & 1.1 & 1.0 & 1.1 & 67.6 & & \\
\hline 50.0 & 1.6 & 1.0 & 1.6 & 69.2 & & \\
\hline 40.0 & 1.3 & 1.0 & 1.3 & 70.5 & & \\
\hline
\end{tabular}

Footage interval 6.0.

Fractures per cubic meter 48 .

Fractures per linear meter 18 . 


\section{APPENDIX C ( cont)}

TA: ${ }^{-1}$ C.-18. Calculation rracture Frequency per Cubic Meter UE $-25 h \# 1$ - 307.0 to $312.0 \mathrm{ft}$

\begin{tabular}{|c|c|c|c|c|c|c|}
\hline Dip & $1 / \cos$ & \# Fract & Product & Sum & Footage & \\
\hline 5.0 & 1.0 & 3.0 & 3.0 & 3.0 & $307.0-308.3$ & broken core \\
\hline 10.0 & 1.0 & 1.0 & 1.0 & 4.0 & & \\
\hline 0.0 & 1.0 & 1.0 & 1.0 & 5.0 & & \\
\hline 30.0 & 1.2 & 1.0 & 1.2 & 6.2 & 308.3 & \\
\hline 75.0 & 3.9 & 1.0 & 3.9 & 10.0 & $308.3-308.7$ & \\
\hline 35.0 & 1.2 & 1.0 & 1.2 & 11.3 & 308.7 & \\
\hline 0.0 & 1.0 & 0.0 & 0.0 & 11.3 & $308.7-309.3$ & waxed core \\
\hline 45.0 & 1.4 & 1.0 & 1.4 & 12.7 & 309.3 & \\
\hline 45.0 & 1.4 & 1.0 & 1.4 & 14.1 & 309.4 & \\
\hline 50.0 & 1.6 & 1.0 & 1.6 & 15.7 & 309.6 & \\
\hline 45.0 & 1.4 & 4.0 & 5.7 & 21.3 & $309.6-310.0$ & \\
\hline 55.0 & 1.7 & 1.0 & 1.7 & 23.1 & 310.0 & \\
\hline 45.0 & 1.4 & 1.0 & 1.4 & 24.5 & $310.0-310.1$ & broken core \\
\hline 10.0 & 1.0 & 1.0 & 1.0 & 25.5 & 310.1 & \\
\hline 60.0 & 2.0 & 1.0 & 2.0 & 27.5 & 310.4 & \\
\hline 50.0 & 1.6 & 1.0 & 1.6 & 29.0 & 310.4 & \\
\hline 30.0 & 1.2 & 1.0 & 1.2 & 30.2 & 310.4 & \\
\hline 55.0 & 1.7 & 1.0 & 1.7 & 31.9 & 310.6 & \\
\hline 45.0 & 1.4 & 1.0 & 1.4 & 33.3 & 310.7 & \\
\hline 15.0 & 1.0 & 1.0 & 1.0 & 34.4 & 311.0 & \\
\hline 45.0 & 1.4 & 1.0 & 1.4 & 35.8 & 311.3 & \\
\hline 50.0 & 1.6 & 1.0 & 1.6 & 37.4 & 311.3 & \\
\hline 5.0 & 1.0 & 1.0 & 1.0 & 38.4 & $311.3-312.0$ & broken core \\
\hline 25.0 & 1.1 & 1.0 & 1.1 & 39.5 & & \\
\hline 55.0 & 1.7 & 1.0 & 1.7 & 41.2 & & \\
\hline 80.0 & 5.8 & 1.0 & 5.8 & 47.0 & & \\
\hline 20.0 & 1.1 & 1.0 & 1.1 & 48.0 & & \\
\hline 75.0 & 3.9 & 1.0 & 3.9 & 51.9 & & \\
\hline
\end{tabular}

Footage interval 5.0.

Fractures der cubic meter 42.

Fractures per 1 inear meter 21 . 
TABLE C-19. Calculation of Fracture Frequency per Cubic Meter $U E-25 h \# 1-312.0$ to $318.0 \mathrm{ft}$

\begin{tabular}{|c|c|c|c|c|c|c|}
\hline Dip & $1 / \cos$ & \# Fract & Product & Sum & Footage & \\
\hline 55.0 & 1.7 & 2.0 & 3.5 & 3.5 & $312.0-312.3$ & broken core \\
\hline 30.0 & 1.2 & 1.0 & 1.2 & 4.6 & & \\
\hline 40.0 & 1.3 & 2.0 & 2.6 & 7.3 & 312.3 & \\
\hline 45.0 & 1.4 & 1.0 & 1.4 & 8.7 & 312.4 & \\
\hline 50.0 & 1.6 & 1.0 & 1.6 & 10.2 & 312.8 & \\
\hline 45.0 & 1.4 & 1.0 & 1.4 & 11.6 & 313.1 & \\
\hline 30.0 & 1.2 & 1.0 & 1.2 & 12.8 & 313.4 & \\
\hline 45.0 & 1.4 & 4.0 & 5.7 & 18.4 & $313.4-313.6$ & broken core \\
\hline 60.0 & 2.0 & 1.0 & 2.0 & 20.4 & & \\
\hline 0.0 & 1.0 & 0.0 & 0.0 & 20.4 & $313.6-314.2$ & waxed core \\
\hline 50.0 & 1.6 & 1.0 & 1.6 & 22.0 & 314.2 & \\
\hline 50.0 & 1.6 & 1.0 & 1.6 & 23.6 & 314.3 & \\
\hline 35.0 & 1.2 & 1.0 & 1.2 & 24.8 & 314.3 & \\
\hline 10.0 & 1.0 & 1.0 & 1.0 & 25.8 & 314.7 & \\
\hline 25.0 & 1.1 & 1.0 & 1.1 & 26.9 & 314.8 & \\
\hline 35.0 & 1.2 & 1.0 & 1.2 & 28.1 & 315.0 & \\
\hline 40.0 & 1.3 & 1.0 & 1.3 & 29.4 & 315.2 & \\
\hline 45.0 & 1.4 & 8.0 & 11.3 & 40.7 & $315.2-316.0$ & lost core \\
\hline 15.0 & 1.0 & 1.0 & 1.0 & 41.8 & $316.0-316.5$ & $40 \%$ recovery \\
\hline 55.0 & 1.7 & 1.0 & 1.7 & 43.5 & & \\
\hline 45.0 & 1.4 & 4.0 & 5.7 & 49.2 & & \\
\hline 20.0 & 1.1 & 1.0 & 1.1 & 50.2 & 316.5 & \\
\hline 40.0 & 1.3 & 1.0 & 1.3 & 51.5 & 316.6 & \\
\hline 30.0 & 1.2 & 1.0 & 1.2 & 52.7 & 316.9 & \\
\hline 55.0 & 1.7 & 1.0 & 1.7 & 54.4 & 316.9 & \\
\hline 10.0 & 1.0 & 1.0 & 1.0 & 55.5 & 317.1 & \\
\hline 25.0 & 1.1 & 1.0 & 1.1 & 56.6 & 317.3 & \\
\hline 0.0 & 1.0 & 1.0 & 1.0 & 57.6 & 317.6 & \\
\hline 15.0 & 1.0 & 1.0 & 1.0 & 58.6 & 317.6 & \\
\hline 30.0 & 1.2 & 1.0 & 1.2 & 59.8 & 318.0 & \\
\hline
\end{tabular}

Footage interval 6.0 .

Fractures per cubic meter 41 .

Fractures der 1 inear meter 24 . 


\section{APPENDIX C (cont)}

TABLE C-20. Calculation of Fracture Frequency per Cubic Meter UE $-25 \mathrm{h \#} 1$ - 318.0 to $323.0 \mathrm{ft}$

\begin{tabular}{|c|c|c|c|c|c|c|}
\hline Dip & $1 / \cos$ & \# Fract & Product & Sum & Footage & \\
\hline 35.0 & 1.2 & 1.0 & 1.2 & 1.2 & $318.0-318.3$ & \\
\hline 50.0 & 1.6 & 1.0 & 1.6 & 2.8 & 318.4 & \\
\hline 60.0 & 2.0 & 1.0 & 2.0 & 4.8 & 318.6 & \\
\hline 45.0 & 1.4 & 1.0 & 1.4 & 6.2 & 318.7 & \\
\hline 0.0 & 1.0 & 1.0 & 1.0 & 7.2 & 318.8 & \\
\hline 15.0 & 1.0 & 1.0 & 1.0 & 8.2 & 318.8 & \\
\hline 10.0 & 1.0 & 1.0 & 1.0 & 9.2 & 318.9 & \\
\hline 10.0 & 1.0 & 1.0 & 1.0 & 10.3 & 319.0 & \\
\hline 25.0 & 1.1 & 1.0 & 1.1 & 11.4 & 319.2 & \\
\hline 0.0 & 1.0 & 1.0 & 1.0 & 12.4 & $319.2-319.9$ & broken core \\
\hline 45.0 & 1.4 & 1.0 & 1.4 & 13.8 & & \\
\hline 35.0 & 1.2 & 1.0 & 1.2 & 15.0 & & \\
\hline 30.0 & 1.2 & 1.0 & 1.2 & 16.1 & & \\
\hline 15.0 & 1.0 & 1.0 & 1.0 & 17.2 & & \\
\hline 40.0 & 1.3 & 1.0 & 1.3 & 18.5 & 319.9 & \\
\hline 85.0 & 11.5 & 1.0 & 11.5 & 30.0 & $319.9-320.0$ & \\
\hline 30.0 & 1.2 & 1.0 & 1.2 & 31.1 & 320.0 & \\
\hline 40.0 & 1.3 & 1.0 & 1.3 & 32.4 & 320.1 & \\
\hline 20.0 & 1.1 & 1.0 & 1.1 & 33.5 & $320.2-320.3$ & \\
\hline 45.0 & 1.4 & 2.0 & 2.8 & 36.3 & & \\
\hline 70.0 & 2.9 & 1.0 & 2.9 & 39.2 & & \\
\hline 45.0 & 1.4 & 1.0 & 1.4 & 40.7 & 320.5 & \\
\hline 50.0 & 1.6 & 1.0 & 1.6 & 42.2 & & \\
\hline 30.0 & 1.2 & 1.0 & 1.2 & 43.4 & 320.6 & \\
\hline 15.0 & 1.0 & 1.0 & 1.0 & 44.4 & 320.7 & \\
\hline 45.0 & 1.4 & 1.0 & 1.4 & 45.8 & $320.7-321.0$ & \\
\hline 70.0 & 2.9 & 1.0 & 2.9 & 48.7 & & \\
\hline 15.0 & 1.0 & 1.0 & 1.0 & 49.8 & 321.0 & \\
\hline 15.0 & 1.0 & 1.0 & 1.0 & 50.8 & 321.1 & \\
\hline 45.0 & 1.4 & 1.0 & 1.4 & 52.2 & $321.1-321.2$ & broken core \\
\hline 25.0 & 1.1 & 1.0 & 1.1 & 53.3 & 321.2 & \\
\hline 50.0 & 2.0 & 1.0 & 2.0 & 55.3 & & \\
\hline 40.0 & 1.3 & 1.0 & 1.3 & 56.6 & 321.7 & \\
\hline 20.0 & 1.1 & 1.0 & 1.1 & 57.7 & 321.9 & \\
\hline 20.0 & 1.1 & 1.0 & 1.1 & 58.8 & 322.3 & \\
\hline 70.0 & 2.9 & 1.0 & 2.9 & 61.7 & 322.3 & \\
\hline 45.0 & 1.4 & 7.0 & 9.9 & 71.6 & $322.3-323.0$ & lost core \\
\hline
\end{tabular}

Footage interval 5.0.

Fractures der cubic meter 58 .

Fractures per linear meter 29. 


\section{APPENDIX C (cont)}

TABLE C-21. Calculation of Fracture Frequency per Cubic Meter UE $-25 h \# 1$ - 333.0 to $337.0 \mathrm{ft}$

\begin{tabular}{|c|c|c|c|c|c|c|}
\hline Dip & $1 / \cos$ & \# Fract & Product & Sum & Footage & \\
\hline 45.0 & 1.4 & 3.0 & 4.2 & 4.2 & $333.0-333.3$ & broken core \\
\hline 0.0 & 1.0 & 1.0 & 1.0 & 5.2 & 333.3 & \\
\hline 45.0 & 1.4 & 1.0 & 1.4 & 6.7 & 333.3 & \\
\hline 80.0 & 5.8 & 1.0 & 5.8 & 12.4 & $333.3-333.5$ & \\
\hline 20.0 & 1.1 & 2.0 & 2.1 & 14.5 & 333.7 & \\
\hline 35.0 & 1.2 & 1.0 & 1.? & 15.8 & 334.0 & \\
\hline 15.0 & 1.0 & 1.0 & 1.0 & 16.8 & 334.4 & \\
\hline 10.0 & 1.0 & 1.0 & 1.0 & 17.8 & 334.6 & \\
\hline 50.0 & 1.6 & 2.0 & 3.1 & 20.9 & $334.6-335.1$ & broken core \\
\hline 40.0 & 1.3 & 1.0 & 1.3 & 22.2 & & \\
\hline 5.0 & 1.0 & 1.0 & 1.0 & 23.2 & & \\
\hline 75.0 & 3.9 & 1.0 & 3.9 & 27.1 & & \\
\hline 85.0 & 11.5 & 1.0 & 11.5 & 38.6 & & \\
\hline 20.0 & 1.1 & 1.0 & 1.1 & 39.6 & 335.1 & \\
\hline 5.0 & 1.0 & 1.0 & 1.0 & 40.6 & 335.8 & \\
\hline 0.0 & 1.0 & 1.0 & 1.0 & 41.6 & & \\
\hline 0.0 & 1.0 & 1.0 & 1.0 & 42.6 & 336.4 & \\
\hline 40.0 & 1.3 & 1.0 & 1.3 & 43.9 & 336.6 & \\
\hline 15.0 & 1.0 & 1.0 & 1.0 & 45.0 & 337.0 & \\
\hline
\end{tabular}

Footage interval 4.0.

Fractures per cubic meter 46 .

Fractures per linear meter 19. 


\section{APPENDIX C (cont)}

TABLE C-22. Calculation of Fracture Frequency per Cubic Meter UE -25 h\# 1 - 337.0 to $343.0 \mathrm{ft}$

\begin{tabular}{|c|c|c|c|c|c|c|}
\hline Dip & $1 / \cos$ & \# Fract & Product & Sum & Footage & \\
\hline 45.0 & 1.4 & 1.0 & 1.4 & 1.4 & $337.0-337.1$ & \\
\hline 45.0 & 1.4 & 1.0 & 1.4 & 2.8 & 337.3 & \\
\hline 10.0 & 1.0 & 1.0 & 1.0 & 3.8 & & \\
\hline 20.0 & 1.1 & 1.0 & 1.1 & 4.9 & 337.8 & \\
\hline 20.0 & 1.1 & 1.0 & 1.1 & 6.0 & 338.3 & \\
\hline 60.0 & 2.0 & 1.0 & 2.0 & 8.0 & 338.3 & \\
\hline 35.0 & 1.2 & 1.0 & 1.2 & 9.2 & 338.4 & \\
\hline 50.0 & 1.6 & 1.0 & 1.6 & 10.7 & 338.6 & \\
\hline 0.0 & 1.0 & 1.0 & 1.0 & 11.7 & 338.6 & \\
\hline 85.0 & 11.5 & 1.0 & 11.5 & 23.2 & $338.6-338.8$ & \\
\hline 25.0 & 1.1 & 1.0 & 1.1 & 24.3 & 338.8 & \\
\hline 0.0 & 1.0 & 0.0 & 0.0 & 24.3 & $339.3-339.9$ & broken core \\
\hline 0.0 & 1.0 & 1.0 & 1.0 & 25.3 & 339.9 & \\
\hline 10.0 & 1.0 & 1.0 & 1.0 & 26.3 & 340.0 & \\
\hline 45.0 & 1.4 & 1.0 & 1.4 & 27.8 & 340.2 & \\
\hline 45.0 & 1.4 & 1.0 & 1.4 & 29.2 & 340.3 & \\
\hline 20.0 & 1.1 & 1.0 & 1.1 & 30.2 & 340.3 & \\
\hline 80.0 & 5.8 & 1.0 & 5.8 & 36.0 & $340.3-340.5$ & \\
\hline 45.0 & 1.4 & 1.0 & 1.4 & 37.4 & 340.5 & \\
\hline 10.0 & 1.0 & 1.0 & 1.0 & 38.4 & 340.5 & \\
\hline 10.0 & 1.0 & 1.0 & 1,0 & 39.4 & 340.8 & \\
\hline 20.0 & 1.1 & 1.0 & 1.1 & 40.5 & 341.2 & \\
\hline 15.0 & 1.0 & 1.0 & 1.0 & 41.5 & 341.4 & \\
\hline 15.0 & 1.0 & 1.0 & 1.0 & 42.6 & 341.6 & \\
\hline 15.0 & 1.0 & 1.0 & 1.0 & 43.6 & 342.2 & \\
\hline 45.0 & 1.4 & 1.0 & 1.4 & 45.0 & 342.2 & \\
\hline 80.0 & 5.8 & 1.0 & 5.8 & 50.8 & $342.2-342.4$ & \\
\hline 45.0 & 1.4 & 1.0 & 1.4 & 52.2 & 342.5 & \\
\hline 60.0 & 2.0 & 1.0 & 2.0 & 54.2 & $342.5-342.7$ & \\
\hline 35.0 & 1.2 & 1.0 & 1.2 & 55.4 & 342.7 & \\
\hline 45.0 & 1.4 & 3.0 & 4.2 & 59.7 & $342.7-343.0$ & \\
\hline
\end{tabular}

Footage interval 6.0.

Fractures per cubic meter 40 . Fractures per linear meter 17 . 
TABLE C-23. Calculation of Fracture Frequency per Cubic Meter UE $-25 h \# 1-343.0$ to $350.0 \mathrm{ft}$

\begin{tabular}{|c|c|c|c|c|c|c|}
\hline Did & $1 / \cos$ & \# Fract & Product & Sum & Footage & \\
\hline 10.0 & 1.0 & 2.0 & 2.0 & 2.0 & $343.0-343.2$ & hairline cracks \\
\hline 0.0 & 1.0 & 1.0 & 1.0 & 3.0 & & \\
\hline 20.0 & 1.1 & 1.0 & 1.1 & 4.1 & & \\
\hline 50.0 & 1.6 & 1.0 & 1.6 & 5.7 & & \\
\hline 45.0 & 1.4 & 1.0 & 1.4 & 7.1 & 343.2 & \\
\hline 45.0 & 1.4 & 2.0 & 2.8 & 9.9 & 343.5 & \\
\hline 5.0 & 1.0 & 1.0 & 1.0 & 10.9 & & \\
\hline 0.0 & 1.0 & 1.0 & 1.0 & 11.9 & 343.7 & \\
\hline 40.0 & 1.3 & 1.0 & 1.3 & 13.2 & 344.0 & \\
\hline 45.0 & 1.4 & 1.0 & 1.4 & 14.6 & 344.0 .344 .8 & broken core \\
\hline 55.0 & 1.7 & 2.0 & 3.5 & 18.1 & & \\
\hline 30.0 & 1.2 & 1.0 & 1.2 & 19.3 & & \\
\hline 50.0 & 1.6 & 1.0 & 1.6 & 20.8 & & \\
\hline 85.0 & 11.5 & 1.0 & 11.5 & 32.3 & & \\
\hline 60.0 & 2.0 & 1.0 & 2.0 & 34.3 & & \\
\hline 40.0 & 1.3 & 1.0 & 1.3 & 35.6 & & \\
\hline 10.0 & 1.0 & 1.0 & 1.0 & 36.6 & 344.8 & \\
\hline 30.0 & 1.2 & 1.0 & 1.2 & 37.8 & 345.0 & \\
\hline 80.0 & 5.8 & 1.0 & 5.8 & 43.5 & $345.0-345.2$ & \\
\hline 25.0 & 1.1 & 1.0 & 1.1 & 44.6 & 345.2 & \\
\hline $45 . C$ & 1.4 & 2.0 & 2.8 & 47.5 & $345.2-345.4$ & broken core \\
\hline 20.0 & 1.1 & 1.0 & 1.1 & 48.5 & 345.4 & ar unen core \\
\hline 45.0 & 1.4 & 1.0 & 1.4 & 49.9 & 345.5 & \\
\hline 30.0 & 1.2 & 1.0 & 1.2 & 51.1 & 345.5 & \\
\hline 55.0 & 1.7 & 1.0 & 1.7 & 52.8 & 345.6 & \\
\hline 15.0 & 1.0 & 1.0 & 1.0 & 53.9 & 345.9 & \\
\hline 45.0 & 1.4 & 6.0 & 8.5 & 62.4 & $345.9-346.5$ & broken core \\
\hline 60.0 & 2.0 & 1.0 & 2.0 & 64.4 & $346.5-346.7$ & \\
\hline 35.0 & 1.2 & 1.0 & 1.2 & 65.6 & 347.0 & \\
\hline 45.0 & 1.4 & 7.0 & 9.9 & 75.5 & $347.0-347.7$ & broken core \\
\hline 45.0 & 1.4 & 22.0 & 31.1 & 106.6 & $347.7-350.0$ & lost core \\
\hline
\end{tabular}




\section{APPENDIX C ( cont)}

TABLE C-24. Calculation of Fracture Frequency per Cubic Meter UE $-25 \mathrm{~h} \# 1-350.0$ to $355.0 \mathrm{ft}$

\begin{tabular}{|c|c|c|c|c|c|c|}
\hline Dip & $1 / \cos$ & \#Fract & Product & Sum & Footage & \\
\hline 50.0 & 1.6 & 1.5 & 2.3 & 2.3 & $350.0-353.0$ & broker core \\
\hline 70.0 & 2.9 & 2.0 & 5.8 & 8.2 & & \\
\hline 10.0 & 1.0 & 2.0 & 2.0 & 10.2 & & \\
\hline 35.0 & 1.2 & 2.0 & 2.4 & 12.7 & & \\
\hline 20.0 & 1.1 & 1.0 & 1.1 & 13.7 & & \\
\hline 15.0 & 1.0 & 1.0 & 1.0 & 14.8 & & \\
\hline 40.0 & 1.3 & 2.0 & 2.6 & 17.4 & & \\
\hline 85.0 & 11.5 & 2.0 & 22.9 & 40.3 & & \\
\hline 80.0 & 5.8 & 1.0 & 5.8 & 46.1 & & \\
\hline 60.0 & 2.0 & 1.0 & 2.0 & 48.1 & & \\
\hline 45.0 & 1.4 & 1.0 & 1.4 & 49.5 & & \\
\hline 20.0 & 1.1 & 1.0 & 1.1 & 50.5 & $353.0-355.0$ & broken core \\
\hline 60.0 & 2.0 & 1.0 & 2.0 & 52.5 & & \\
\hline 45.0 & 1.4 & 1.0 & 1.4 & 54.0 & & \\
\hline 0.0 & 1.0 & 1.0 & 1.0 & 55.0 & & \\
\hline 50.0 & 1.6 & 1.0 & 1.6 & 56.5 & & \\
\hline 15.0 & 1.0 & 1.0 & 1.0 & 57.6 & & \\
\hline 35.0 & 1.2 & 1.0 & 1.2 & 58.8 & & \\
\hline 25.0 & 1.1 & 1.0 & 1.1 & 59.9 & & \\
\hline 55.0 & 1.7 & 1.0 & 1.7 & 61.6 & & \\
\hline
\end{tabular}

Footage interval 5.0.

Fractures per cubic meter 50 .

Fractures per linear meter 17 . 


\section{APPENDIX C (cont)}

TABLE C-25. Calculation of Fracture Frequency per Cubic Meter UE-25h\#1 - 355.0 to $363.0 \mathrm{ft}$

\begin{tabular}{|c|c|c|c|c|c|c|}
\hline Dip & $1 / \cos$ & \# Fract & Product & Sum & Footage & \\
\hline 85.0 & 11.5 & 2.0 & 22.9 & 22.9 & $355.0-356.7$ & broken core \\
\hline 35.0 & 1.2 & 1.0 & 1.2 & 24.2 & & \\
\hline 45.0 & 1.4 & 3.0 & 4.2 & 28.4 & & \\
\hline 0.0 & 1.0 & 1.0 & 1.0 & 29.4 & & \\
\hline 70.0 & 2.9 & 1.0 & 2.9 & 32.3 & & \\
\hline 50.0 & 1.6 & 1.0 & 1.5 & 33.9 & & \\
\hline 15.0 & 1.0 & 1.0 & 1.0 & 34.9 & & \\
\hline 80.0 & 5.8 & 1.0 & 5.8 & 40.7 & & \\
\hline 60.0 & 2.0 & 1.0 & 2.0 & 42.7 & $356.7-357.1$ & \\
\hline 5.0 & 1.0 & 1.0 & 1.0 & 43.7 & 357.1 & \\
\hline 85.0 & 11.5 & 1.0 & 11.5 & 55.2 & $357.1-357.4$ & \\
\hline 50.0 & 1.6 & 1.0 & 1.6 & 56.7 & 357,4 & \\
\hline 50.0 & 1.6 & 3.0 & 4.7 & 61.4 & $357.4-359.5$ & broken core \\
\hline 60.0 & 2.0 & 2.0 & 4.0 & 65.4 & & \\
\hline 45.0 & 1.4 & 1.0 & 1.4 & 66.8 & & \\
\hline 85.0 & 11.5 & 2.0 & 22.9 & 89.7 & & \\
\hline 15.0 & 1.0 & 1.0 & 1.0 & 90.8 & & \\
\hline 55.0 & 1.7 & 1.0 & 1.7 & 92.5 & & \\
\hline 30.0 & 1.2 & 1.0 & 1.2 & 93.7 & & \\
\hline 70.0 & 2.9 & 1.0 & 2.9 & 96.6 & & \\
\hline 65.0 & 2.4 & 1.0 & 2.4 & 99.0 & & \\
\hline 10.0 & 1.0 & 1.0 & 1.0 & 100.0 & 359.5 & \\
\hline 40.0 & 1.3 & 1.0 & 1.3 & 101.3 & 359.5 & \\
\hline 40.0 & 1.3 & 1.0 & 1.3 & 102.6 & 359.6 & \\
\hline 45.0 & 1.4 & 2.0 & 2.8 & 105.4 & $359.6-359.8$ & broken core \\
\hline 0.0 & 1.0 & 1.0 & 1.0 & 106.4 & 359.8 & \\
\hline 70.0 & 2.9 & 1.0 & 2.9 & 109.3 & 359.8 & \\
\hline 20.0 & 1.1 & 1.0 & 1.1 & 110.4 & 360.0 & \\
\hline 35.0 & 1.2 & 1.0 & 1.2 & 111.6 & $360.0-360.1$ & broken core \\
\hline 0.0 & 1.0 & 1.0 & 1.0 & 112.6 & & \\
\hline 85.0 & 11.5 & 1.0 & 11.5 & 124.1 & $360.1-360.3$ & \\
\hline 10.0 & 1.0 & 1.0 & 1.0 & 125.1 & 360.3 & \\
\hline 40.0 & 1.3 & 1.0 & 1.3 & $126 . \overline{4}$ & 360.6 & \\
\hline 0.0 & 1.0 & 1.0 & 1.0 & 127.4 & 360.7 & \\
\hline 45.0 & 1.4 & 1.0 & 1.4 & 128.8 & 360.8 & \\
\hline 85.0 & 11.5 & 1.0 & 11.5 & 140.3 & $360.7-360.9$ & \\
\hline 40.0 & 1.3 & 1.0 & 1.3 & 141.6 & 360.9 & \\
\hline 45.0 & 1.4 & 2.5 & 3.5 & 145.2 & $360.9-362.3$ & broken core \\
\hline 25.0 & 1.1 & 2.0 & 2.2 & 147.4 & & \\
\hline 20.0 & 1.1 & 1.0 & 1.1 & 148.4 & & \\
\hline $\begin{array}{l}40.0 \\
85.0\end{array}$ & $\begin{array}{r}1.3 \\
11.5\end{array}$ & 1.0 & $\begin{array}{r}1.3 \\
229\end{array}$ & 149.7 & & \\
\hline $\begin{array}{r}85.0 \\
5.0\end{array}$ & $\begin{array}{r}11.5 \\
1.0\end{array}$ & $\begin{array}{l}2.0 \\
1.0\end{array}$ & $\begin{array}{r}22.9 \\
1.0\end{array}$ & $\begin{array}{l}172.7 \\
173.7\end{array}$ & 362.3 & \\
\hline 40.0 & 1.3 & 1.0 & 1.3 & 175.0 & 362.5 & \\
\hline 45.0 & 1.4 & 4.0 & 5.7 & 180.6 & $362.5-363.0$ & lost core \\
\hline
\end{tabular}

Footage interval 8.0.

Fractures per cubic meter 92.

Fractures per linear meter 24. 
TABLE C-26. Calculation of Fracture Frequency per Cubic Meter UE $-25 \mathrm{~h} \# 1-363.0$ to $368.0 \mathrm{ft}$

\begin{tabular}{|c|c|c|c|c|c|c|}
\hline Did & $1 / \cos$ & \# Fract & Product & Sum & Foctage & \\
\hline 60.0 & 2.0 & 2.0 & 4.0 & 4.0 & $363.0-363.3$ & broken core \\
\hline 55.0 & 1.7 & 1.0 & 1.7 & 5.7 & & \\
\hline 15.0 & 1.0 & 1.0 & 1.0 & 6.8 & & \\
\hline 55.0 & 1.7 & 1.0 & 1.7 & 8.5 & 363.3 & \\
\hline 40.0 & 1.3 & 1.0 & 1.3 & 9.8 & 363.6 & \\
\hline 45.0 & 1.4 & 1.0 & 1.4 & 11.2 & 363.8 & \\
\hline 40.0 & 1.3 & 1.0 & 1.3 & 12.5 & 364.0 & \\
\hline 70.0 & 2.9 & 1.0 & 2.9 & 15.5 & 364.0 & \\
\hline 55.0 & 1.7 & 1.0 & 1.7 & 17.2 & 364.2 & \\
\hline 10.0 & 1.0 & 1.0 & 1.0 & 18.2 & 364.3 & \\
\hline 35.0 & 1.2 & 1.0 & 1.2 & 19.5 & 365.1 & \\
\hline 5.0 & 1.0 & 1.0 & 1.0 & 20.5 & 365.1 & \\
\hline 45.0 & 1.4 & 1.0 & 1.4 & 21.9 & $365.2-365.3$ & \\
\hline 55.0 & 1.7 & 1.0 & 1.7 & 23.6 & & \\
\hline 0.0 & 1.0 & 1.0 & 1.0 & 24.6 & & \\
\hline 40.0 & 1.3 & 1.0 & 1.3 & 25.9 & & \\
\hline 50.0 & 1.6 & 1.0 & 1.6 & 27.5 & 365.5 & \\
\hline 45.0 & 1.4 & 1.0 & 1.4 & 28.9 & 365.7 & \\
\hline 50.0 & 1.6 & 1.0 & 1.6 & 30.4 & 365.9 & \\
\hline 45.0 & 1.4 & 6.0 & 8.5 & 38.9 & $365.9-366.6$ & broken core \\
\hline 81.0 & 6.4 & 1.0 & 6.4 & 45.3 & & \\
\hline 55.0 & 1.7 & 2.0 & 3.5 & 48.8 & 366.6 & \\
\hline 30.0 & 1.2 & 1.0 & 1.2 & 50.0 & 366.9 & \\
\hline 50.0 & 1.6 & 2.0 & 3.1 & 53.1 & 367.3 & \\
\hline 60.0 & 2.0 & 1.0 & 2.0 & 55.1 & 367.5 & \\
\hline 20.0 & 1.1 & 1.0 & 1.1 & 56.1 & 367.8 & \\
\hline 70.0 & 2.9 & 1.0 & 2.9 & 59.1 & $367.8-368.0$ & broken core \\
\hline 10.0 & 1.0 & 1.0 & 1.0 & 60.1 & & \\
\hline
\end{tabular}

Footage interval 5.0 .

Fractures per cubic meter 49 .

Fractures per 1 inear meter 24 . 
TABLE C-27. Calculation of Fracture Frequency per Cubic Meter UE $-25 h \# 1-368.0$ to $372.0 \mathrm{ft}$

\begin{tabular}{|c|c|c|c|c|c|c|}
\hline Dip & $1 / \cos$ & \# Fract & Product & Sum & Footage & \\
\hline 45.0 & 1.4 & 1.0 & 1.4 & 1.4 & $368.0-368.1$ & broken core \\
\hline 25.0 & 1.1 & 1.0 & 1.1 & 2.5 & 368.1 & \\
\hline 55.0 & 1.7 & 1.0 & 1.7 & 4.3 & 368.1 & \\
\hline 55.0 & 1.7 & 1.0 & 1.7 & 6.0 & 368.3 & \\
\hline 80.0 & 5.8 & 1.0 & 5.8 & 11.8 & 368.4 & \\
\hline 45.0 & 1.4 & 1.0 & 1.4 & 13.2 & $368.4-368.5$ & broken core \\
\hline 30.0 & 1.2 & 1.0 & 1.2 & 14.3 & 368.5 & \\
\hline 15.0 & 1.0 & 1.0 & 1.0 & 15.4 & 368.6 & \\
\hline 55.0 & 1.7 & 1.0 & 1.7 & 17.1 & 368.8 & \\
\hline 5.0 & 1.0 & 1.0 & 1.0 & 18.1 & 368.8 & \\
\hline 85.0 & 11.5 & 1.0 & 11.5 & 29.6 & $368.8-369.0$ & \\
\hline 15.0 & 1.0 & 1.0 & 1.0 & 30.6 & $369.0-370.2$ & broken core \\
\hline 10.0 & 1.0 & 1.0 & 1.0 & 31.6 & & \\
\hline 0.0 & 1.0 & 2.0 & 2.0 & 33.6 & & \\
\hline 25.0 & 1.1 & 1.0 & 1.1 & 34.7 & & \\
\hline 5.0 & 1.0 & 1.0 & 1.0 & 35.7 & & \\
\hline 70.0 & 2.9 & 1.0 & 2.9 & 38.7 & & \\
\hline 55.0 & 1.7 & 1.0 & 1.7 & 40.4 & & \\
\hline 45.0 & 1.4 & 1.0 & 1.4 & 41.8 & & \\
\hline 20.0 & 1.1 & 1.0 & 1.1 & 42.9 & $370.2-370.5$ & \\
\hline 85.0 & 11.5 & 1.0 & 11.5 & 54.4 & & \\
\hline 25.0 & 1.1 & 1.0 & 1.1 & 55.5 & & \\
\hline 20.0 & 1.1 & 1.0 & 1.1 & 56.5 & 370.6 & \\
\hline 20.0 & 1.1 & 1.0 & 1.1 & 57.6 & 370.7 & \\
\hline 50.0 & 1.6 & 1.0 & 1.6 & 59.2 & $370.7-371.1$ & \\
\hline 30.0 & 1.2 & 1.0 & 1.2 & 60.3 & 371.1 & \\
\hline 60.0 & 2.0 & 1.0 & 2.0 & 62.3 & $371.1-371.3$ & \\
\hline 40.0 & 1.3 & 1.0 & 1.3 & 63.6 & 371.3 & \\
\hline 25.0 & 1.1 & 1.0 & 1.1 & 64.7 & 371.5 & \\
\hline 55.0 & 1.7 & 1.0 & 1.7 & 66.5 & 371.5 & \\
\hline 85.0 & 11.5 & 1.0 & 11.5 & 77.9 & $371.5-371.7$ & \\
\hline 10.0 & 1.0 & 3.0 & 3.0 & 81.0 & $371.7-372.0$ & broken $\mathrm{co}$ \\
\hline
\end{tabular}

Footage interval 4.0 .

Fractures per cubic meter 82 .

Fractures per linear meter 29. 


\section{APPENDIX C (cont)}

TABLE C-28. Calculation of Fracture Frequency per Cubic Meter UE $-25 \mathrm{~h} \# 1-372.0$ to $378.0 \mathrm{ft}$

\begin{tabular}{|c|c|c|c|c|c|c|}
\hline Dip & $1 / \cos$ & \# Fract & Product & Sum & Footage & \\
\hline 10.0 & 1.0 & 4.0 & 4.1 & 4.1 & $372.0-372.4$ & \\
\hline 45.0 & 1.4 & 1.0 & 1.4 & 5.5 & 372.4 & \\
\hline 0.0 & 1.0 & 1.0 & 1.0 & 6.5 & 372.6 & \\
\hline 0.0 & 1.0 & 1.0 & 1.0 & 7.5 & 372.9 & \\
\hline 20.0 & 1.1 & 1.0 & 1.1 & 8.5 & 373.0 & \\
\hline 0.0 & 1.0 & 1.0 & 1.0 & 9.5 & 373.2 & \\
\hline 5.0 & 1.0 & 1.0 & 1.0 & 10.5 & 373.3 & \\
\hline 30.0 & 1.2 & 1.0 & 1.2 & 11.7 & 373.4 & \\
\hline 0.0 & 1.0 & 1.0 & 1.0 & 12.7 & 373.5 & \\
\hline 0.0 & 1.0 & 1.0 & 1.0 & 13.7 & 373.8 & \\
\hline 85.0 & 11.5 & 1.0 & 11.5 & 25.2 & $373.8-374.1$ & broken core \\
\hline 0.0 & 1.0 & 1.0 & 1.0 & 26.2 & & \\
\hline 50.0 & 1.6 & 1.0 & 1.6 & 27.7 & & \\
\hline 20.0 & 1.1 & 1.0 & 1.1 & 28.8 & 374.1 & \\
\hline 50.0 & 1.6 & 1.0 & 1.6 & 30.3 & 374.1 & \\
\hline 0.0 & 1.0 & 1.0 & 1.0 & 31.3 & 374.4 & \\
\hline 40.0 & 1.3 & 2.0 & 2.6 & 34.0 & 374.9 & \\
\hline 0.0 & 1.0 & 1.0 & 1.0 & 35.0 & 375.1 & \\
\hline 80.0 & 5.8 & 1.0 & 5.8 & 40.7 & $375.1-375.3$ & \\
\hline 40.0 & 1.3 & 1.0 & 1.3 & 42.0 & & \\
\hline 20.0 & 1.1 & 1.0 & 1.1 & 43.1 & & \\
\hline 0.0 & 1.0 & 1.0 & 1.0 & 44.1 & & \\
\hline 30.0 & 1.2 & 1.0 & 1.2 & 45.2 & 375.3 & \\
\hline 10.0 & 1.0 & 1.0 & 1.0 & 46.3 & 375.7 & \\
\hline 45.0 & 1.4 & 1.0 & 1.4 & 47.7 & 375.8 & \\
\hline 35.0 & 1.2 & 1.0 & 1.2 & 48.9 & 375.8 & \\
\hline 65.0 & 2.4 & 1.0 & 2.4 & 51.3 & 376.1 & \\
\hline 20.0 & 1.1 & 1.0 & 1.1 & 52.3 & 376.1 & \\
\hline 45.0 & 1.4 & 1.0 & 1.4 & 53.7 & $376.1-376.2$ & broken core \\
\hline 60.0 & 2.0 & 1.0 & 2.0 & 55.7 & 376.3 & \\
\hline 55.0 & 1.7 & 1.0 & 1.7 & 57.5 & 376.6 & \\
\hline 10.0 & 1.0 & 1.0 & 1.0 & 58.5 & 376.6 & \\
\hline 45.0 & 1.4 & 1.0 & 1.4 & 59.9 & 376.7 & \\
\hline 35.0 & 1.2 & 1.0 & 1.2 & 61.1 & 377.0 & \\
\hline 85.0 & 11.5 & 1.0 & 11.5 & 72.6 & $377.0-377.3$ & \\
\hline 15.0 & 1.0 & 1.0 & 1.0 & 73.6 & 377.3 & \\
\hline 10.0 & 1.0 & 2.0 & 2.0 & 75.7 & 377.5 & \\
\hline 80.0 & 5.8 & 1.0 & 5.8 & 81.4 & $377.5-377.7$ & \\
\hline 5.0 & 1.0 & 1.0 & 1.0 & 82.4 & 377.7 & \\
\hline 5.0 & 1.0 & 1.0 & 1.0 & 83.4 & $377.7-378.0$ & broken core \\
\hline 45.0 & 1.4 & 2.0 & 2.8 & 86.3 & & \\
\hline
\end{tabular}

Footage interval 6.0.

Fractures per cubic meter 59 .

Fractures per linear meter $2 \overline{6}$. 


\section{APPENDIX C (cont)}

TABLE C-29. Calculation of Fracture Frequency per Cubic Meter UE $-25 h \# 1-378.0$ to $388.0 \mathrm{ft}$

\begin{tabular}{|c|c|c|c|c|c|c|}
\hline Dip & $1 / \cos$ & \# Fract & Product & Sum & Footage & \\
\hline 25.0 & 1.1 & 1.0 & 1.1 & 1.1 & 378.0 & \\
\hline 10.0 & 1.0 & 1.0 & 1.0 & 2.1 & 378.3 & \\
\hline 45.0 & 1.4 & 1.0 & 1.4 & 3.5 & 378.5 & \\
\hline 85.0 & 11.5 & 1.0 & 11.5 & 15.0 & $378.5-378.9$ & \\
\hline 40.0 & 1.3 & 1.0 & 1.3 & 16.3 & 378.9 & \\
\hline 10.0 & 1.0 & 1.0 & 1.0 & 17.3 & 379.3 & \\
\hline 0.0 & 1.0 & 0.0 & 0.0 & 17.3 & $379.3-380.0$ & waxed core \\
\hline 10.0 & 1.0 & 1.0 & 1.0 & 18.3 & 380.0 & \\
\hline 45.0 & 1.4 & 1.0 & 1.4 & 19.8 & 380.2 & \\
\hline 60.0 & 2.0 & 1.0 & 2.0 & 21.8 & $380.2-380.4$ & \\
\hline 25.0 & 1.1 & 1.0 & 1.1 & 22.9 & 380.7 & \\
\hline 30.0 & 1.2 & 1.0 & 1.2 & 24.0 & 380.9 & \\
\hline 40.0 & 1.3 & 1.0 & 1.3 & 25.3 & 380.9 & \\
\hline 25.0 & 1.1 & 1.0 & 1.1 & 26.4 & 381.1 & \\
\hline 0.0 & 1.0 & 1.0 & 1.0 & 27.4 & 381.3 & \\
\hline 0.0 & 1.0 & 1.0 & 1.0 & 28.4 & 381.5 & \\
\hline 35.0 & 1.2 & 1.0 & 1.2 & 29.6 & 381.7 & \\
\hline 70.0 & 2.9 & 1.0 & 2.9 & 32.6 & $382.3-382.8$ & \\
\hline 10.0 & 1.0 & 1.0 & 1.0 & 33.6 & 383.3 & \\
\hline 15.0 & 1.0 & 1.0 & 1.0 & 34.6 & 383.8 & \\
\hline 50.0 & 1.6 & 1.0 & 1.6 & 36.2 & 383.8 & \\
\hline 10.0 & 1.0 & 1.0 & 1.0 & 37.2 & 384.0 & \\
\hline 40.0 & 1.3 & 1.0 & 1.3 & 38.5 & 384.2 & \\
\hline 35.0 & 1.2 & 1.0 & 1.2 & 39.7 & 384.2 & \\
\hline 20.0 & 1.1 & 1.0 & 1.1 & 40.8 & 384.3 & \\
\hline 55.0 & 1.7 & 1.0 & 1.7 & 42.5 & 384.5 & \\
\hline 20.0 & 1.1 & 1.0 & 1.1 & 43.6 & 384.5 & \\
\hline 55.0 & 1.7 & 1.0 & 1.7 & 45.3 & 384.5 & \\
\hline 20.0 & 1.1 & 1.0 & 1.1 & 46.4 & 384.5 & \\
\hline 55.0 & 1.7 & 1.0 & 1.7 & 48.1 & 385.3 & \\
\hline 20.0 & 1.1 & 1.0 & 1.1 & 49.2 & 385.3 & \\
\hline 55.0 & 1.7 & 1.0 & 1.7 & 50.9 & 385.6 & \\
\hline 45.0 & 1.4 & 3.0 & 4.2 & 55.2 & $385.6-385.9$ & \\
\hline 55.0 & 1.7 & 1.0 & 1.7 & 56.9 & 385.9 & \\
\hline 30.0 & 1.2 & 1.0 & 1.2 & 58.1 & 385.9 & \\
\hline 15.0 & 1.0 & 1.0 & 1.0 & 59.1 & 386.1 & \\
\hline 50.0 & 1.6 & 1.0 & 1.6 & 60.7 & 386.1 & \\
\hline 20.0 & 1.1 & 1.0 & 1.1 & 61.7 & 386.3 & \\
\hline 55.0 & 1.7 & 1.0 & 1.7 & 63.5 & 386.8 & \\
\hline 20.0 & 1.1 & 1.0 & 1.1 & 64.6 & 386.8 & \\
\hline 20.0 & 1.1 & 1.0 & 1.1 & 65.6 & 387.1 & \\
\hline 0.0 & 1.0 & 0.0 & 0.0 & 65.6 & $387.1-387.4$ & waxed core \\
\hline 0.0 & 1.0 & 1.0 & 1.0 & 66.6 & 387.4 & \\
\hline 5.0 & 1.0 & 1.0 & 1.0 & 67.6 & 387.5 & \\
\hline 45.0 & 1.4 & 1.0 & 1.4 & 69.0 & 387.9 & \\
\hline 30.0 & 1.2 & 1.0 & 1.2 & 70.2 & 388.0 & \\
\hline
\end{tabular}

Footage interval 10.0.

Fractures per cubic meter 29.

Fractures per linear meter 15. 


\section{¿}

4 the

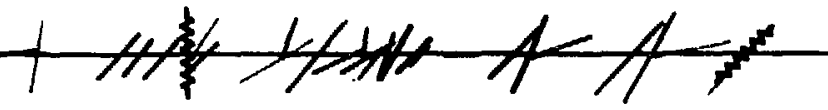

H4t

LEGEND

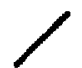

PLANAR SMOOTH FRACTURE NO MINERAL COATING (SHOWN ONLY IN FIRST 200 FEET)

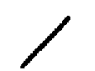

MANGANESE OXIDES (Mn Ox) COATED FRACTURE
FAULT, AS INDICATED

BY BRECCIA
CALCITE OR CALICHE $\left(\mathrm{CaCO}_{3}\right)$ VEINLET FILLING FRACTURE

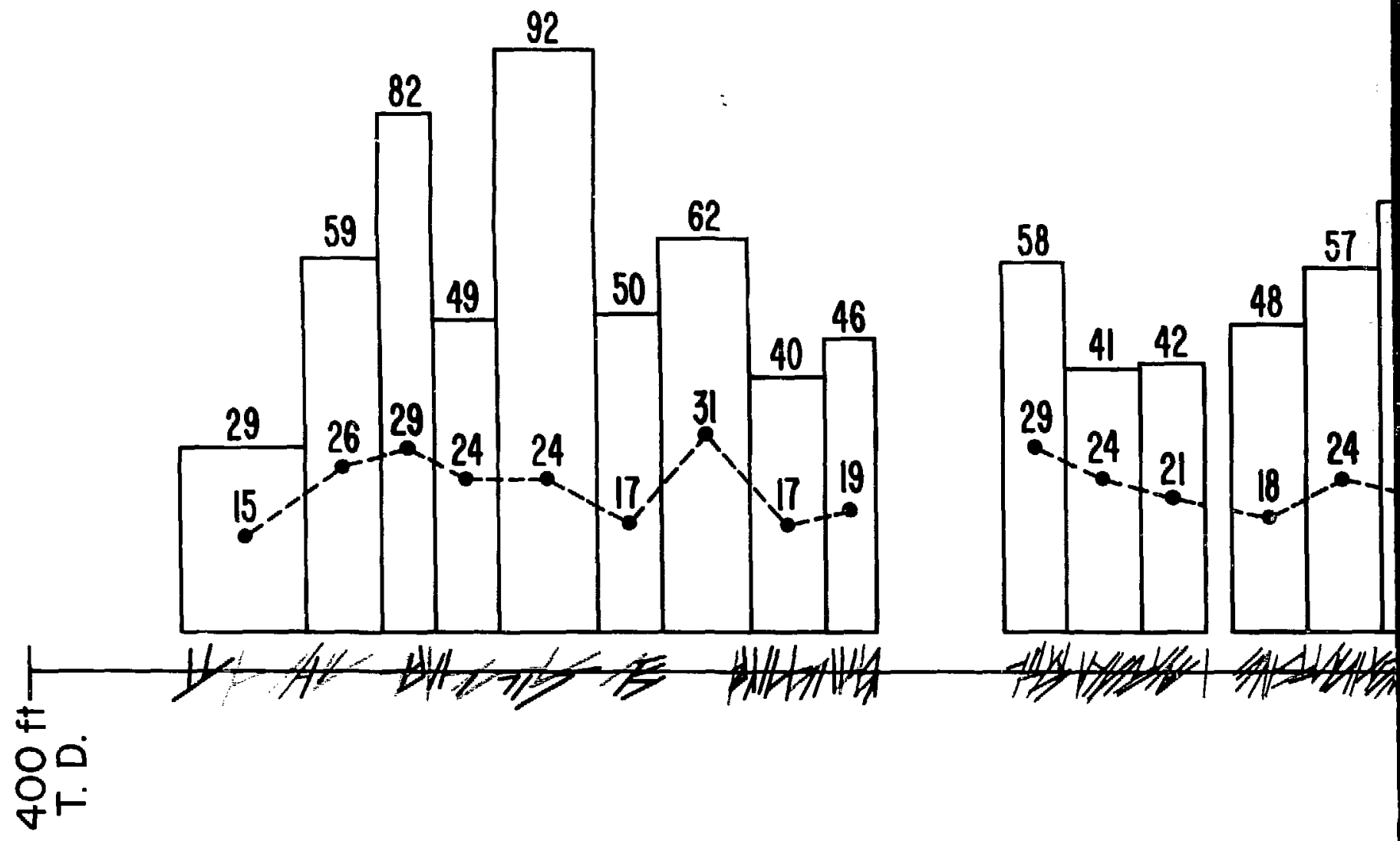
deviation). In absence of oriented core, angle of west, except for second fracture that intersects angle west may actually dip east. For explanati Footages with no graphs had significant core los: (see Appendix C). Moreover, not all nonmineralizec (see Appendix $C$ ), but rearly all mineralized fract 
